Studying photosynthetic antenna complexes in vivo and in vitro using time-resolved fluorescence spectroscopy

\section{Reza Ranjbar Choubeh}

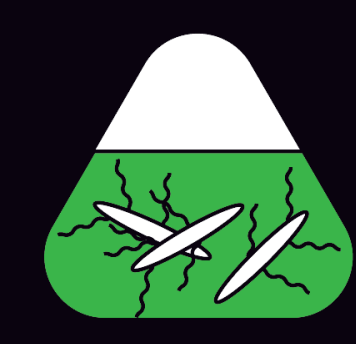

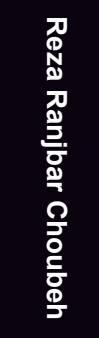

2018
Invitation

you are eindry invited to

attend the pubfic defence of my PhD thesis

studying photosynthetic antenna comprexes in vivo and in vitro using time-resofved fluorescence spectroscopy spectroscopy

on Monday, 19th of the aula of Wageningen (Genral Foulkesweg ia)

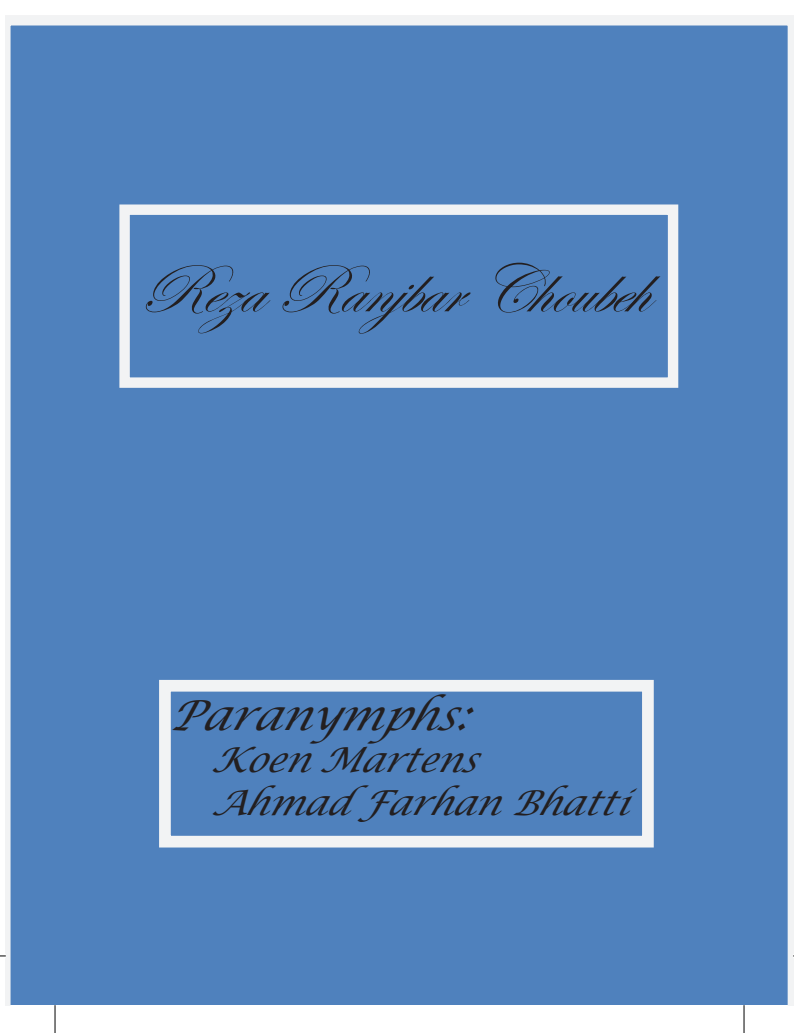




\section{Propositions}

1. The scale of state II quenching of PSII in cyanobacteria depends on the way state II is induced and on what cyanobacterium strain is used.

(this thesis)

2. Desert dwelling cyanobacteria avoid photodamage under drought by the reversible loss of phycobilisome's structural organization.

(this thesis)

3. Universities should record the courses they provide their students with and make them accessible to the general public.

4. Pursuit of beauty and elegance in a mathematical theory of nature should not be the driving force in physics.

5. If all universities join to invest in software development and journal publishing, they get both software and access right to scientific literature far cheaper than is currently the case, while the scientific community and general public will enjoy free software and literature.

6. If open-offices were any good, more senior staff would enjoy them too.

Propositions belonging to the thesis, entitled

Studying photosynthetic antenna complexes in vivo and in vitro using time-resolved fluorescence spectroscopy

Reza Ranjbar Choubeh

Wageningen, 19 November 2018 
Studying photosynthetic antenna complexes in vivo and in vitro using time-resolved fluorescence spectroscopy 


\section{Thesis committee}

\section{Promotors}

Prof. Dr Herbert van Amerongen

Professor of Biophysics

Wageningen University \& Research

Prof. Dr Paul C. Struik

Professor of Crop physiology

Wageningen University \& Research

\section{Other members}

Prof. Dr Frans A.M. Leermakers, Wageningen University \& Research

Prof. Dr Alfred R. Holzwarth, Max-Planck-Institute for Chemical

Energy Conversion, Mülheim an der Ruhr, Germany

Dr Ivo H.M. van Stokkum, VU, Amsterdam

Dr Jeremy Harbinson, Wageningen University \& Research

This research was conducted under the auspices of the Graduate School EPS (Experimental Plant Sciences). 


\title{
Studying photosynthetic antenna complexes in vivo and in vitro using time-resolved fluorescence spectroscopy
}

\author{
Reza Ranjbar Choubeh
}

\author{
Thesis \\ submitted in fulfilment of the requirements for \\ the degree of doctor \\ at Wageningen University \\ by the authority of the Rector Magnificus, \\ Prof. Dr A.P.J. Mol, \\ in the presence of the \\ Thesis Committee appointed by the Academic Board to \\ be defended in public \\ on Monday 19 November 2018 \\ at 4 p.m. in the Aula.
}


Reza Ranjbar Choubeh

Studying photosynthetic antenna complexes in vivo and in vitro using timeresolved fluorescence spectroscopy, 182 pages.

PhD thesis, Wageningen University, Wageningen, the Netherlands (2018) With references, with summary in English

ISBN: 978-94-6343-495-9

DOI: https://doi.org/10.18174/457749 


\section{Contents}

Chapter 1

Chapter 2

Chapter 3

Chapter 4

Chapter 5

Chapter 6

Chapter 7
Introduction

Picosecond excitation energy transfer of allophycocyanin studied in solution and in crystals 33

State transitions in the cyanobacterium Synechococcus elongatus 7942 involve reversible quenching of the photosystem II core 57

Light harvesting dynamics in desert crust cyanobacteria: Changes in aggregation state as a mechanism for modulating energy transfer

Efficiency of excitation-energy trapping in the reaction center of the low-light adapted green photosynthetic bacterium Chlorobaculum tepidum

General discussion 163

Summary 175

Acknowledgements 179 
1

Introduction 


\section{Background}

Photosynthesis is the main source of energy for almost all life on earth. Photosynthetic organisms live under variable conditions, which demands short-term adaptation to avoid damage to photosynthetic antennas. The main topic of this thesis is the study of the variation of light harvesting in different physiological conditions in cyanobacteria Synechococcus elongatus 7942 and Leptolyngbya ohadii.

In this introduction, early steps of photosynthesis and some of the photosynthetic complexes are explained and then an introduction of fluorescence and its acquisition is presented. Data analysis that is necessary to make sense of the recorded timeresolved fluorescence follows next. At the end, the organization of this thesis is given.

\section{Photosynthesis, cyanobacteria, and state transitions}

Photosynthetic organisms convert light energy into chemical energy to sustain their metabolism, but which can also be used by other living organisms. Photosynthetic reactions are divided into light-dependent and light-independent reactions (Blankenship 2008a). Plants and cyanobacteria produce Adenosine Triphosphate (ATP) and reduced nicotinamide adenine dinucleotide phosphate (NADPH) during the light-dependent reactions. In the light-independent reactions, ATP and NADPH are used to fix carbon dioxide into carbohydrates.

The light-dependent reactions start with the absorption of light by the photosynthetic antennas. Cyanobacteria use phycobilisomes (PBSs) and photosystems I and II (PSI and PSII) to absorb light (Figure 1). PBSs are the main light-harvesting antennas in cyanobacteria which mainly absorb green-orange light. They are composed of rodlike proteins and core proteins. (MacColl 2004; Mirkovic et al. 2016). The rods are built of C-phycocyanins (CPCs) and in various organisms they also have phycoerythrins (PEs). The rods are attached to allophycocyanins (APCs), which contain APC trimers and three other protein-pigments, namely $L_{C M}, \beta^{16}$, and $\alpha^{B}$. The excitation energy absorbed by PEs and PCs travels along the rods and reaches APCs, which are attached to the rods by linker proteins, and from there it is delivered 
to $L_{C M}$ and $\alpha^{B}$. $L_{C M}$ and $\alpha^{B}$ transfer the excitation energy to chlorophyll $(\mathrm{Chl})$ a of PSI and PSII.

Figure 2 shows a schematic representation of a PBS; the structures of CPC and APC are also shown. In the PBS, CPC and APC form hexameric and trimeric structures in which a monomer consists of $\alpha$ - and $\beta$-subunits. The $\alpha$ - and $\beta$-subunits both bind bilin pigments. In CPC (APC) the $\alpha$ - and $\beta$-subunits contain one and two (one) bilins, respectively. These hexamers and trimers in turn stack together to form rods or cylinders.

These pigment-protein complexes are organized as an energetic funnel with many high-energy pigments at the periphery and far less low-energy pigments in the center of the PBS close to the PSs where the excitation should be delivered. CPC hexamers have an absorption and emission peak at $624 \mathrm{~nm}$ and $651 \mathrm{~nm}$ (Holzwarth et al. 1987). APC trimers have an absorption and emission peak at $650 \mathrm{~nm}$ and $660 \mathrm{~nm}$ (MacColl 2004). Lcm and $\alpha^{B}$ have an emission peak at $680 \mathrm{~nm}$. The cyanobacterium Leptolyngbya ohadii, which resides in the desert, performs photosynthesis in the early morning just after sunrise as long as the temperature is not high yet and the dew deposited before dawn is still available. After some time however, Leptolyngbya ohadii starts quenching the excitation energy, transforming it into heat during the main part of the day to prevent damage to its photosynthetic complexes and in this thesis, we will demonstrate that this quenching happens at the level of the PBS. 


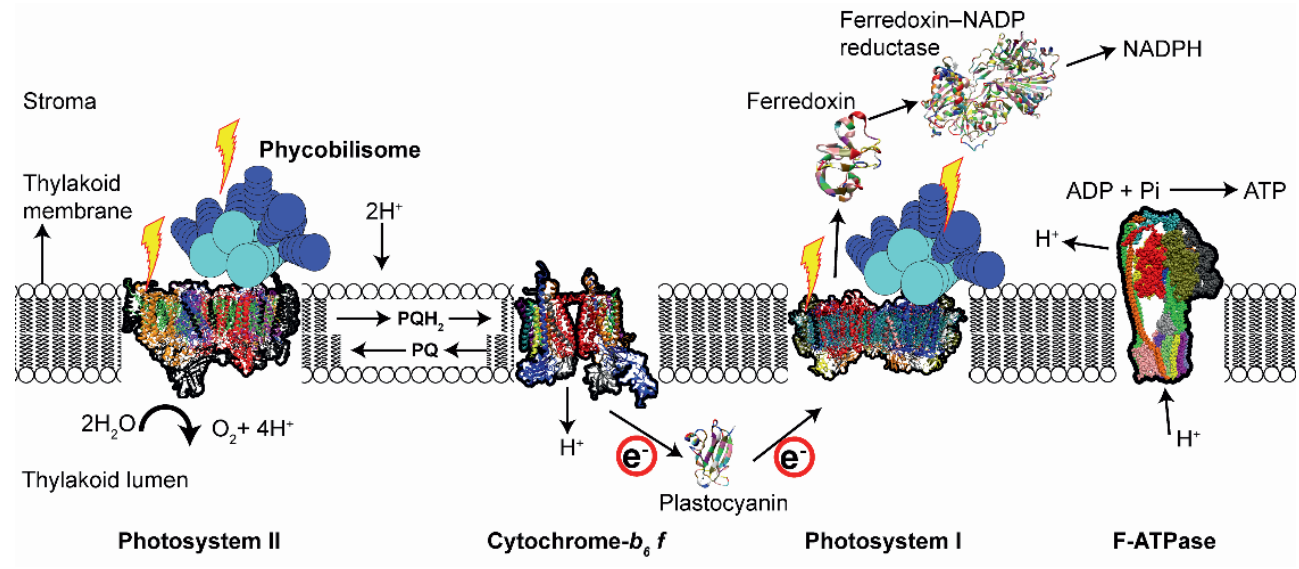

Figure 1. Schematic representation of the light reactions in cyanobacteria showing how the different photosynthetic units work together (Nelson and Ben-Shem 2004; Blankenship 2008b). The straight arrows in PSI indicate the electron transport pathway. The image does not show the real size of complexes relative to each other, nor does it represent the real relative distances between them.

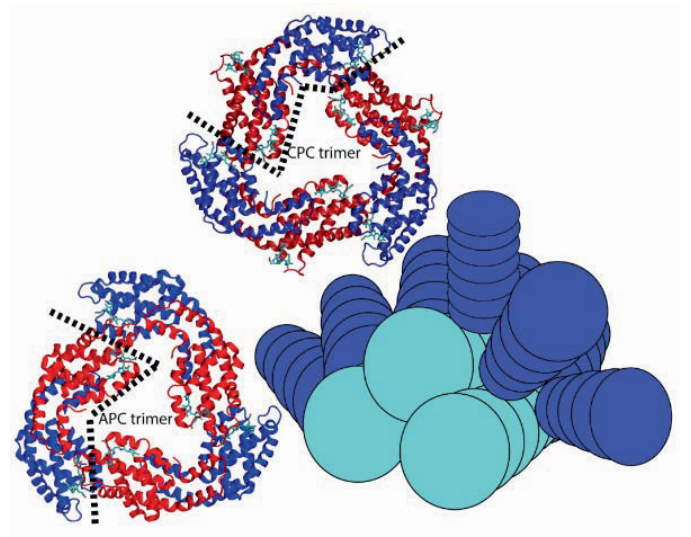

Figure 2. Schematic representation of a PBS (bottom right). The CPC rods and APC are represented in blue and cyan, respectively. The trimeric forms of CPC and APC are also shown separately. The $\alpha$ - and $\beta$-subunits are shown in blue and red, respectively. The bilins are shown in cyan. The dotted line separates one monomer. The codes in the protein data bank are $1 \mathrm{jbo}$ (CPC) and 4rmp (APC).

PSII is another multi-subunit complex present in cyanobacteria, which is generally believed to form dimers in vivo (Nelson and Ben-Shem 2004; Nickelsen and Rengstl 2013; Zlenko et al. 2017) (Figure 3). The PSII reaction center complex largely consists of two proteins, D1 and D2 and binds six chlorophylls a, two pheophytins a 
and two quinone molecules. There are two light-harvesting antennas, CP43 and CP47, attached to the PSII reaction center. The light energy absorbed by PBSs, CP43 and CP47 is transferred to the PSIl reaction center (RC) (Ferreira et al. 2004; Nickelsen and Rengstl 2013; Mirkovic et al. 2016). In the RC there are two different pathways that both result in the same charge-separated state with the electron residing on a pheophytin molecule (Novoderezhkin et al.; Romero et al. 2010) (Figure 4). The electron is then donated to a quinone called $Q_{A}$, which in turn donates an electron to a mobile quinone called $Q_{B}$ (see Figure 4 ). The reduction of $Q_{B}\left(Q_{B}{ }^{-1}\right)$ occurs twice and each time it is reduced

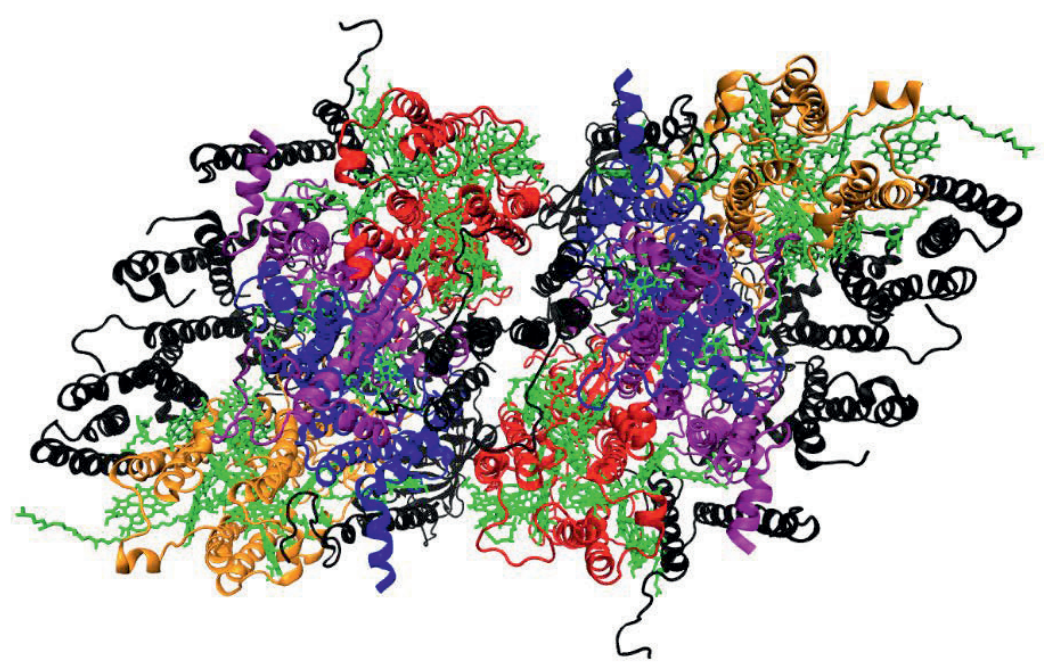

Figure 3. Top view of a PSII dimer. Chls a are shown in green. CP43 and CP47 are shown in orange and red, respectively. D1 and D2 are shown in blue and purple, respectively. Other protein complexes are shown in black. The protein data bank code is $1 \mathrm{~s} 5 \mathrm{l}$. 


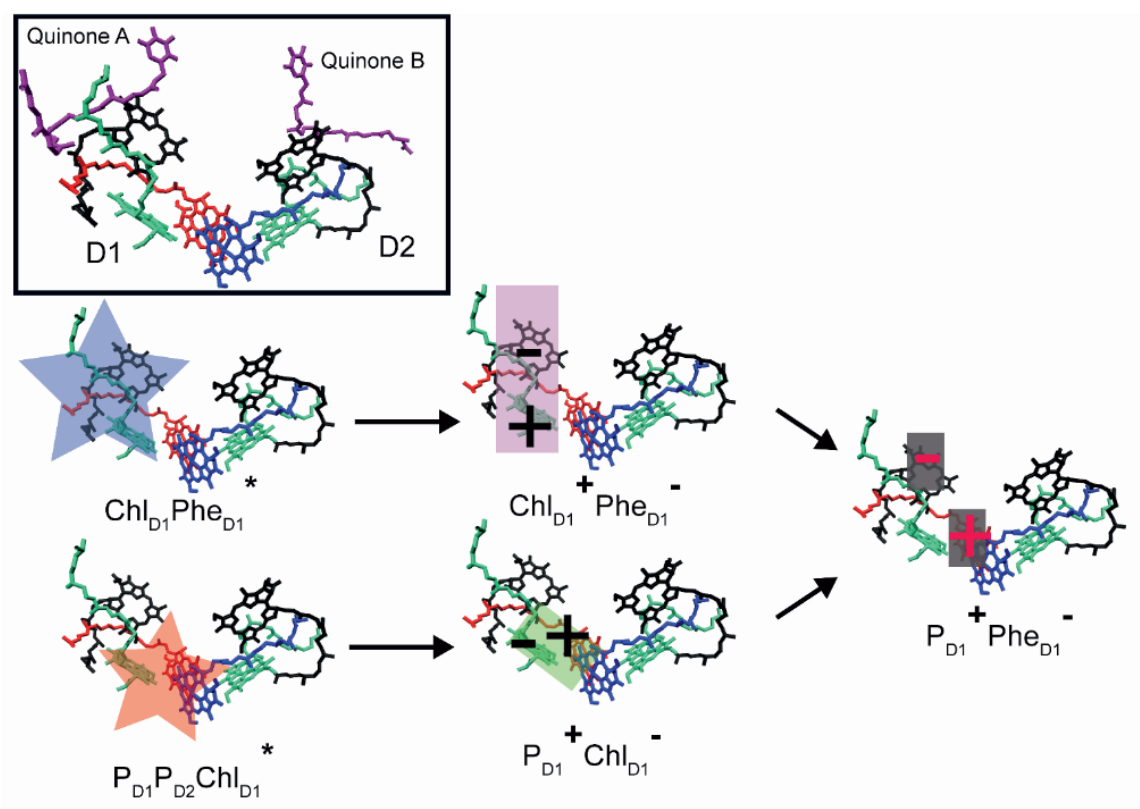

Figure 4. The cofactors involved in electron transport in PSII. The figure in the box shows four Chl a, two pheophytin and two quinone molecules as described as follows. $P_{D 1}$ and $P_{D 2}$, which are Chls $a$, are shown in red and blue and next to them are two other Chls $a$ in green, which are called ChI $\mathrm{D}_{\mathrm{D}}$ and $\mathrm{Chl}_{\mathrm{D} 2}$. The pheophytin molecules $\left(\mathrm{Phe}_{\mathrm{D} 1}\right.$ and $\left.\mathrm{Phe}_{\mathrm{D} 2}\right)$ are represented in black and the quinones in purple. The bottom of the figure shows two different pathways that result in the same chargeseparated state. Only one branch is active in charge separation. The star shows the excited state and the rectangles show the charge-separated states.

it takes up one proton from the stroma (two in total) to finally form $Q_{B} H_{2}$. $Q_{B} H_{2}$ is then released and is replaced by an oxidized quinone.

The released $\mathrm{Q}_{\mathrm{B}} \mathrm{H}_{2}$ (plastoquinone) reaches the cytochrome- $b_{6} f$ complex (Figure 5). Cytochrome- $b_{6} f$ occurs in dimeric form (Nelson and Ben-Shem 2004) and each monomer has, among other smaller subunits, four major subunits namely cytochrome $f$, cytochrome- $b_{6}$, the Rieske iron-sulphur protein, and subunit IV (Figure 5). Cytochrome $f$ and the Rieske iron-sulphur protein are involved in the transfer of one electron from reduced quinone to plastocyanin, which later donates its electron to the PSI reaction center (P700). Cytochrome- $b_{6}$, through its two haem groups (bL and $\mathrm{b}_{\mathrm{H}}$ ), is involved in translocating one electron across the membrane to reduce a quinone at the stromal $Q_{i}$ site. This quinone reduction event happens twice at the stromal $Q_{i}$ site. The reduced quinone then takes up two protons and is released into 12 
the bilipid membrane. The oxidization of plastoquinone by cytochrome- $b_{6} f$ complex and water splitting by PSII create a proton motive force across the membrane. The protons pass from the lumen to the stroma through the F-ATP synthase, which is a protein embedded in the thylakoid membrane. F-ATP synthase then adds a phosphate to Adenosine diphosphate (ADP) to make Adenosine triphosphate (ATP) (Nelson and Ben-Shem 2004).

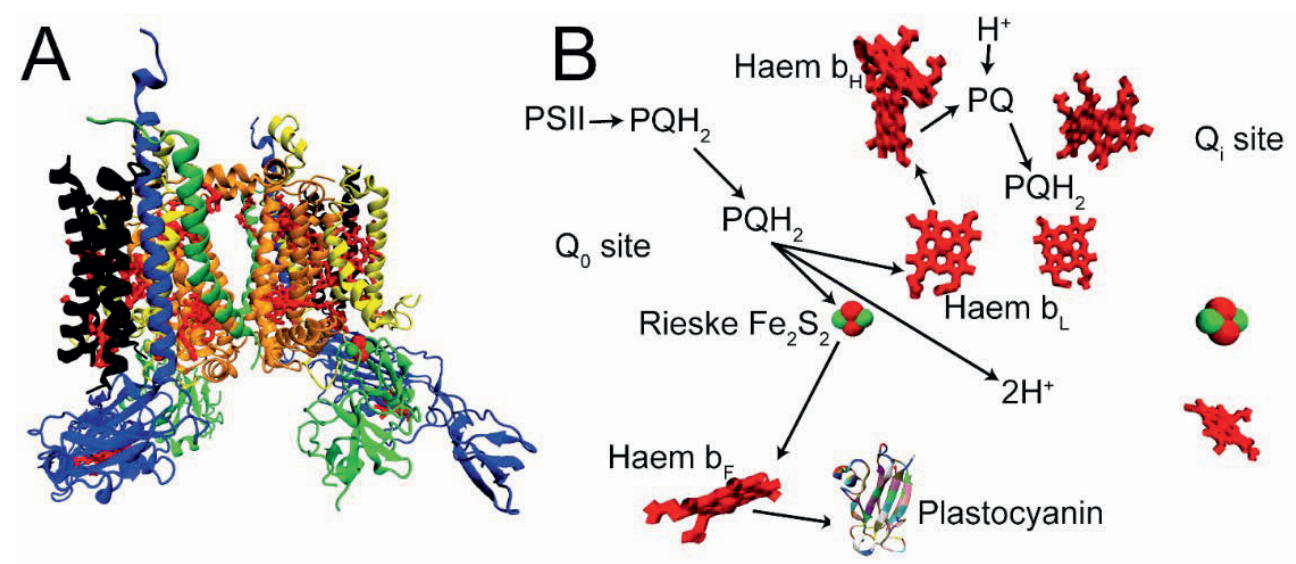

Figure 5. The protein structure of a dimer of cytochrome- $b_{6} f$ complex is shown with A) the four major subunits called cytochrome $f$ (blue), cytochrome- $b_{6}$ (orange), Rieske iron-sulphur (green), and subunit IV (yellow). Other minor subunits are shown in black. B) all the protein structures are removed to show the electron transport chain in cytochrome- $b_{6} f$ complex. The protein data bank code is $1 \mathrm{q} 90$.

PSI is another multi-subunit complex in cyanobacteria that works in series with PSII and occurs in trimeric form in vivo (monomeric in plants) (Figure 6). The PSI reaction center contains a chlorophyll pair called P700 (Figure 7), which is the primary electron donor that leads to light-driven charge separation. In the PSI reaction center core there are other molecules which transfer the electron donated by the excited P700 to ferredoxin. The PSI reaction center is surrounded by $\sim 90 \mathrm{Chl}$ a molecules that act as a light-harvesting antenna and are bound by the PSI core (Nelson and Ben-Shem 2004).

Depending on the illumination conditions PSI and PSII receive different amounts of light energy, leading to different yields of charge separation. An imbalance in rate of charge separation between PSI and PSII manifests itself in the ratio of reduced and 
oxidized quinone (the redox state of the quinone pool) (Mullineaux and Allen 1990). The process by which the cyanobacteria attempt to restore the balance between PSI and PSII is called state transition (Kirilovsky 2015) and this process has been studied in Chapter 3. It is found that in Synechococcus elongatus 7942 the imbalance in excitation of PSI and PSII in state II, when the quinone pool is more reduced, is compensated by the quenching of excitation energy in PSII.

Time- and spectrally-resolved fluorescence can provide information about the excited-state population of different photosynthetic complexes and the excitation energy transfer (EET) pathway within and among these complexes. The excitedstate population depends on the conditions to which the photosynthetic organisms are adapted and the measurements may provide insight in various adaptation mechanisms. This will be explained in more detail below.

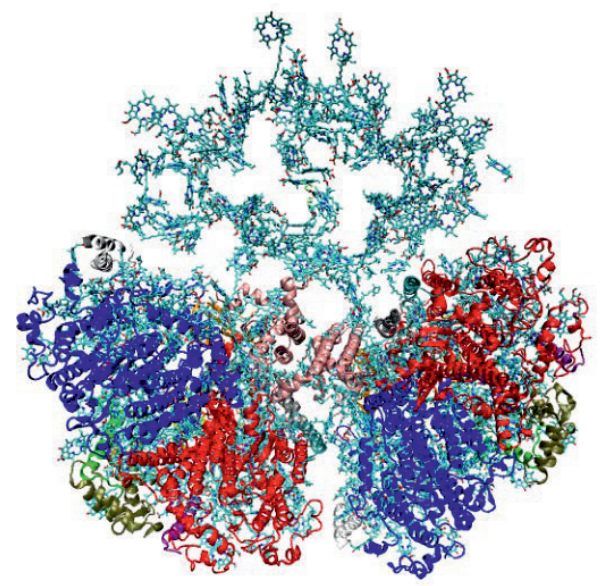

Figure 6. The trimeric structure of cyanobacterial PSI. There are two major subunits shown in blue (psaA) and red (psaB) that contain the $\mathrm{P} 700 \mathrm{Chl}$ a pair. The proteins are removed from one of the monomers to clearly show the $\mathrm{Chl}$ a molecules. The code in the protein data bank is $1 \mathrm{jb0}$. 


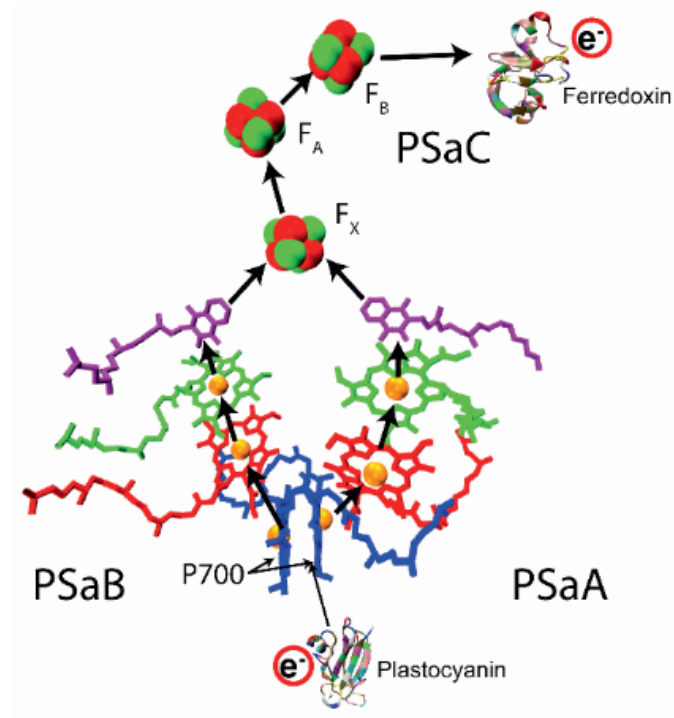

Figure 7. P700 of PSI along with other molecules that transfer electrons from plastocyanin to ferredoxin. The P700 special pair of $\mathrm{Chl}$ a molecules is shown in blue and the $\mathrm{Mg}$ atoms in orange. Other Chls a are shown in red and green. The purple molecules are quinones. The red and green spheres show the $\mathrm{Fe}_{4} \mathrm{~S}_{4}$ cluster. The electron transport pathway is indicated by arrows (Nelson and Ben-Shem 2004).

\section{Green sulfur bacteria}

So far, we explained the major photosynthetic complexes in cyanobacteria. Other photosynthetic bacteria such as green sulfur bacteria have a different lightharvesting complex, which is much larger than PBS, PSI, or PSII. In Chapter 5, the efficiency of EET in Chlorobacalum tepidum is studied in whole cells at $77 \mathrm{~K}$ and room temperature. This section presents a short introduction to the photosynthetic antennas of green sulfur bacteria.

Green sulfur bacteria are photosynthetic bacteria that do not produce oxygen during photosynthesis and do not split water to get protons and electrons. Instead, they use $\mathrm{H}_{2} \mathrm{~S}$ or other sulfuric compounds as the primary reductant. $\mathrm{C}$. tepidum belongs to this group of bacteria, and it is studied in this thesis. The largest light-harvesting antennas of green sulfur bacteria are the chlorosomes. On the outside chlorosomes are encapsulated by a lipid monolayer and inside they contain self-aggregates of bacteriochlorophyll (BChl) $c, d$, or $e$ (only one type of $\mathrm{BChl}$ per species) and a smaller 
amount of carotenoids and quinones. Chlorosomes have a typical length of 100-200 $\mathrm{nm}$ and a width of $10-60 \mathrm{~nm}$. They contain in the order of $10^{5} \mathrm{BChls}$, which are highly organized (Frese et al. 1997)in tubular-lamellar structures (for a review see (Oostergetel et al. 2010)).

The excitation energy captured by a chlorosome is transferred to its baseplate, which consists of pigment-protein complexes containing $\mathrm{BChl} a$ and they are embedded in the lipid monolayer surrounding the chlorosomes.

The baseplate transfers its excitation energy to the Fenna-Matthews-Olson protein (FMO) complexes. These pigment-protein complexes form trimers and each monomer contains 8 BChls a (see Figure 8).

The excitation energy finally reaches one of the RCs. In green sulfur bacteria $2 \mathrm{FMO}$ trimers are associated with one reaction center (Permentier et al. 2000; Hauska et al. 2001). It has also been proposed that four FMO trimers are associated with 2 RCs in a supercomplex (Bína et al. 2016). The RC core of green sulfur bacteria contains 16 BChls a, 4 Chls a, and 2 carotenoids (Hauska et al. 2001). The primary electron donor is a closely bound pair of BChls a called P840. The excited P840 donates an electron to a $\mathrm{Chl}$ a molecule. This electron, through other intermediary molecules, reduces a water soluble ferredoxin protein. The oxidized special pair is re-reduced by a bound cytochrome $c_{551}$. The electron that cytochrome $c_{551}$ donates to the oxidized special pair originates from a sulfur compound such as $\mathrm{H}_{2} \mathrm{~S}$. 


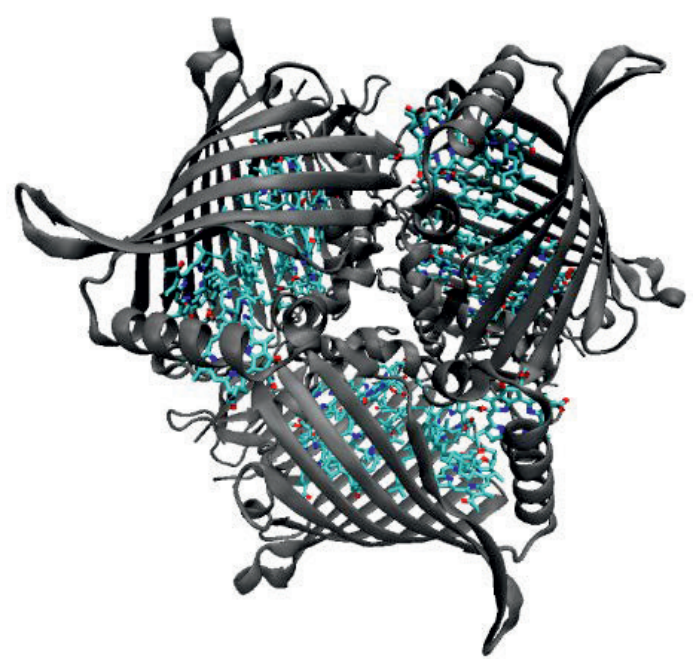

Figure 8. Molecular structure of FMO isolated from C. tepidum. FMO is trimeric and each monomer contains $8 \mathrm{BChls}$ a. The protein data bank code is 3eni.

\section{Fluorescence}

Fluorescence is the emission of electromagnetic radiation, mainly in the UV and visible region that originates from an atomic or molecular excited singlet state, in which the total spin of all the electrons in the molecule is zero (Lakowicz 2006). Fluorophores, which absorb light at a specific wavelength and re-emit it at longer wavelengths, have multiple higher electronic states. Each electronic state is associated with many vibrational states. After absorption of light, a fluorophore is excited to $S_{1}$ (first electronic state) or a higher electronic state. Usually, the excited molecules relax to the first electronic state and the ensemble of the excited molecules have a Boltzmann distribution among the vibrational states associated with the first electronic state. This relaxation process is called internal conversion (IC) and typically occurs within $\sim 1$ ps or less (Lakowicz 2006). The fluorescence lifetime itself is typically $\sim 1-10 \mathrm{~ns}$ and generally fluorescence occurs from a thermally equilibrated excited state (Lakowicz 2006). Sometimes the singlet excited state transforms to a triplet excited state in which the total spin for the excited molecule is one. This process is called intersystem crossing (ISC) and may lead to phosphorescence. The triplet state has a relatively long lifetime which is on the order of microseconds for carotenoids and milliseconds for Chls (Peterman et al. 1995). 
When the photosynthesis process is saturated in high light, triplet state formation will become the dominant process. Chl triplets are extremely dangerous for the organisms, and they may lead to the formation of highly reactive singlet oxygen. Most Chls are protected by carotenoids, which can scavenge the triplets and thereby prevent photodamage. However, this is not true for the Chls in the RCs and to minimize photodamage in adverse conditions, PSII will often be protected by the activation of non-photochemical quenching processes which release excitation energy as heat.

The fluorescence lifetime is often far shorter than 1-10 ns due to additional processes such as EET to other molecules, followed by charge separation in the RC. This EET occurs from one molecule to another via radiationless transfer processes (van Amerongen et al. 2000). Figure 9 shows the processes described above in a Jablonski diagram. The absorption of light is shown by the upward solid blue arrow while the emission of light is shown by a downward solid red arrow. IC, ISC, and EET are shown by the dotted black arrows while the wavy brown line indicates vibrational relaxation.

Fluorescence provides information about the excited-state population and the photosynthetic complexes involved in EET. However, to obtain this information usually mathematical modeling of the raw data is needed. In the next section, timeresolved data acquisition is explained and after that the data analysis of the obtained data is presented. 


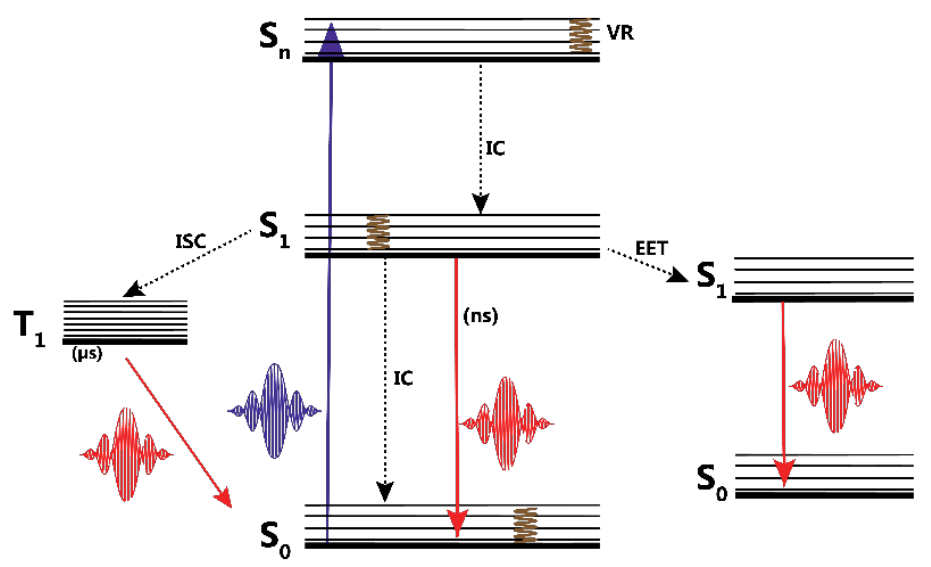

Figure 9. A Jablonski diagram that summarizes various excited-state transitions in or between photosynthetic pigments (see the above text for further explanation)

\section{Time-resolved fluorescence spectroscopy}

A streak-camera system was used to perform time-resolved fluorescence spectroscopy. A schematic representation of a streak-camera system is shown in Figure 10. In summary, the laser excitation light induces fluorescence in the sample.

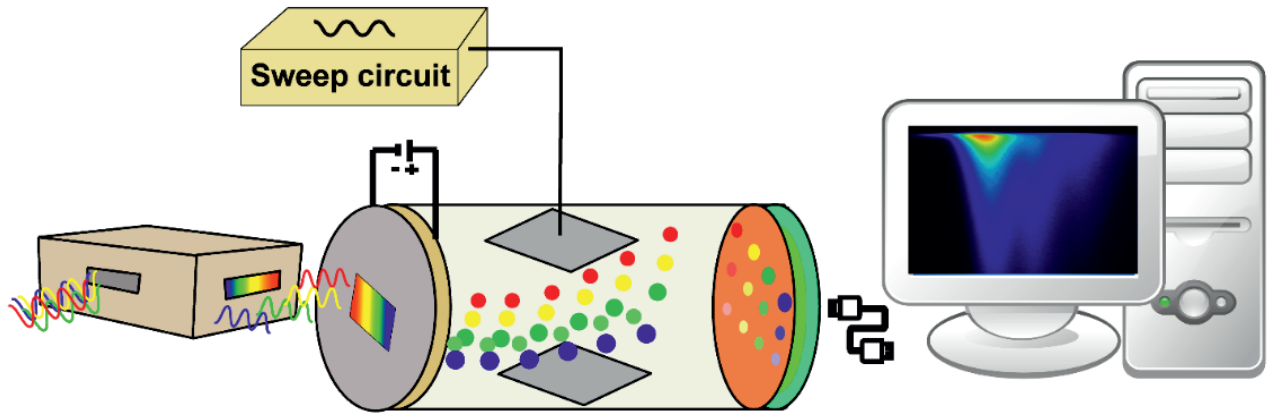

Figure 10. Schematic representation of a streak camera. For further explanation see text.

The fluorescence is collected through a lens and is projected onto a spectrograph. In the spectrograph, the fluorescence is dispersed over a range of wavelengths in the horizontal direction. The dispersed light hits a photocathode that produces electrons with energies corresponding to the wavelength (energy) of the fluorescence light. The photoelectrons then are accelerated in an accelerating mesh. 
The accelerated photoelectrons pass through a varying electric field and are deflected in the vertical direction. The photoelectrons that arrive at different times experience different electric fields and are deflected at different angles. The photoelectrons that now are dispersed horizontally (corresponding to the energy of the photon that produced them) and vertically (corresponding to their arrival time) hit a microchannel plate (MCP) and are multiplied several thousand times and reach a phosphor screen. The photoelectrons that strike the phosphor screen produce photons that are collected and focused on a CCD detector. The three-dimensional data (time, wavelength, and number of photons) is then stored for further processing.

The detection efficiency of the streak camera depends on the wavelength of the detected light and the position on the phosphor screen/CCD detector. To correct for the uneven detection of light, the light of a reference lamp must be recorded by the streak camera. The obtained data of the samples must then be divided by the image of the reference lamp and the result must be multiplied by the steady state spectrum of the lamp, which should be measured separately.

After a laser pulse excites a sample, it starts to fluorescence. The detected light is the convolution of the time-dependent fluorescence with the time-dependent instrument response function (IRF). In case of a streak-camera system, the IRF can be modeled as a Gaussian function or the sum of several Gaussian functions each with their own central position and full-width at half-maximum (FWHM). The detected signal is (Lakowicz 2006; van Stokkum et al. 2008; van Oort et al. 2009):

$$
\begin{aligned}
& F(t, \lambda)=\int_{0}^{t} \operatorname{IRF}(t-x, \lambda) * I(x, \lambda) \mathrm{d} x \quad \text { eq. } 1 \\
& I R F(t, \lambda)=\sum_{i=1}^{n} \frac{\sqrt{4 \ln (2)}}{\Delta \sqrt{\pi}} e^{-4 \ln (2) \times\left(\frac{t-\mu}{\Delta}\right)^{2}} \text { eq. } 2
\end{aligned}
$$

In eq. $1, t$ is the time at which a photon is detected. $\lambda$ is wavelength and represents the wavelength-dependence of the fluorescence $I(x, \lambda) . x$ is the integration variable and represents time. IRF is wavelength-dependent too because its central position $\mu$ (eq. 2 ) in time depends on wavelength. $F(t, \lambda)$ is the detected fluorescence signal. 
In eq. 2, the IRF used for a streak camera is shown. $n$ is the used number of Gaussian functions and $\Delta$ is the FWHM.

The integration of fluorescence over time yields the fluorescence steady-state spectrum.

\section{Data Analysis}

When a pigment that is "surrounded" by many other pigments is excited, it can transfer its excitation energy to its neighbors and via a random walk the excitation energy reaches one of the RCs (van Amerongen et al. 2000). We model the EET among spectrally distinguishable pigments by first order differential equations (Loefroth 1986; van Stokkum et al. 2004): Consider two groups of pigments named $a$ and $b$ (Figure 11). Initially, only pigments a are excited and a population of 1 is assigned to them (the total population of excited state is normalized to 1). Pigments a transfer their energy to pigments $b$ with a rate $k_{a b} . k_{a}$ and $k_{b}$ are the rates of nonradiative loss of excitation energy. Pigments $b$ lose the majority of their excitation energy by the transfer of their excitation to the RC (not shown) with a rate $k_{b}$, which is a non-radiative process. For pigments $a, k_{a}$ is any non-radiative process that quenches the excitation energy.

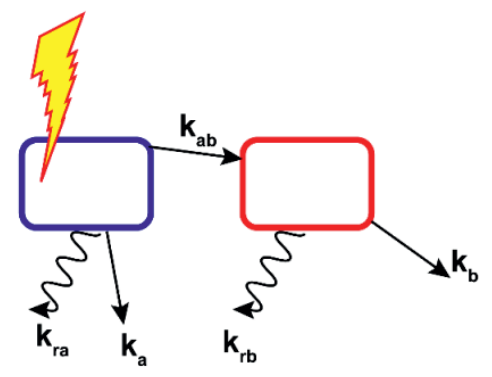

Figure 11. A compartmental model of two groups of pigments. The blue and red compartments represent the excited-state populations of pigments $a$ and $b$. Initially only pigments $a$ are populated, but over time they irreversibly transfer some of their excitation energy to pigments $b$. To obtain a simple analytical solution for demonstration purposes, the EET from pigments $b$ to $a$ is neglected here. $\boldsymbol{k}_{r a}$ and $\boldsymbol{k}_{r b}$ represent the radiative rates of each group of pigments. $\boldsymbol{k}_{a b}$ is the rate of EET from pigments $a$ to $b . k_{a}$ and $k_{b}$ are the rate of non-radiative loss of the excitation energy. 
Pigments $a$ and $b$ decay due to fluorescence with rates of $k_{r a}$ and $k_{r b}$, respectively. The population of excitation energy over time can be obtained by solving the equations below:

$$
\begin{aligned}
& \frac{d}{d t} a(t)=-\left(k_{a b}+k_{r a}+k_{a}\right) a(t) \quad \text { eq. } 3 \\
& \frac{d}{d t} b(t)=-\left(k_{b}+k_{r b}\right) b(t)+k_{a b} a(t)
\end{aligned}
$$

The solution is:

$$
\begin{aligned}
a(t) & =e^{-\left(k_{a b}+k_{r a}+k_{a}\right) t} e q .4 \\
b(t) & =-\frac{k_{a b}}{k_{a}+k_{a b}-k_{b}+k_{r a}-k_{r b}} e^{-\left(k_{a b}+k_{r a}+k_{a}\right) t} \\
& \quad+\frac{k_{a b}}{k_{a}+k_{a b}-k_{b}+k_{r a}-k_{r b}} e^{-\left(k_{b}+k_{r b}\right) t}
\end{aligned}
$$

Notice that if there was no energy transfer between pigments $a$ and $b$, i.e. $k_{a b}=0$, then the excited state of pigment a would decay with a rate of $k_{r a}+k_{a}$, which is the rate of fluorescence plus all other non-radiative process. However, due to EET the excited state of pigment a decays with a rate of $k_{a b}+k_{r a}+k_{a}$. The plot of the population over time is shown in Figure $12 \mathrm{~A}$ with the rates $k_{r a}=k_{r b}=$ $(1000 p s)^{-1}, k_{a b}=(50 p s)^{-1}, k_{b}=(200 p s)^{-1}, k_{a}=0$. These rates are typical rates (in terms of order of magnitude) encountered in photosynthetic complexes.

The fluorescence over time and wavelength is modelled as the multiplication of the population of each group of pigments over time by each group of pigment's spectral emission over wavelength and also their respective radiative rate (Loefroth 1986; van Stokkum et al. 2004).

$$
I(\lambda, t)=k_{r a} f_{a}(\lambda) \times \mathrm{a}(t)+k_{r b} f_{b}(\lambda) \times \mathrm{b}(t) \text { eq. } 5
$$

Snapshots of fluorescence emission are shown in Figure 12B for different times. The rates used are the same as the ones used for Figure 12A. The spectral emission shape of pigments $a$ and $b$ is assumed to be Gaussian (on a wavelength scale) with centers at $650 \mathrm{~nm}$ and $700 \mathrm{~nm}$, respectively, and full width half maximum (FWHM) of $15 \mathrm{~nm}$ for both pigments. 

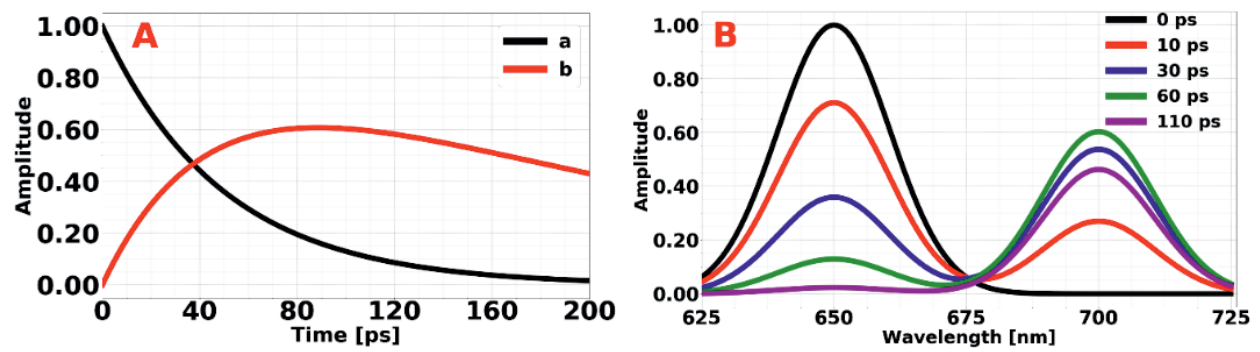

Figure 12. A) Excited-state population of two groups of pigments. Initially only pigments a are excited, but then as time proceeds, the excited-state energy is transferred to pigments $b$. B) The fluorescence spectra of pigments $a$ and $b$ at different times. At $t=0$, only pigments a fluoresce. While the exited state energy is transferred to pigments $b$ over time, the fluorescence of pigments $b$ rises and later it starts to decrease.

At time zero only pigments $a$ are fluorescing and the emission from pigments $b$ is zero. After 10 ps part of the excitation energy has been transferred from pigments a to pigments $b$ and the emission of the latter is visible at $\sim 700 \mathrm{~nm}$. At 30-60 ps more excitation energy has arrived at pigments $b$ and its emission amplitude has become larger. At 110 ps the emission of pigments $b$ has decreased again because of transfer to the reaction centers.

Equations 3-5 form the basis for what is called target analysis with the model shown in Figure 11. Fitting eq. 5 to the experimental data yields the unknown rates and the spectra of pigments in the model of Figure 11. .

It is not clear beforehand if the model introduced in Figure 11 can describe the measured data well. For example, it might not be known whether there is EET from pigments $b$ to $a$. To obtain insight from experimental data without paying attention to the details of the model, it is necessary to perform a global data analysis. Using eq. 4-5 we get:

$$
\begin{array}{r}
I(\lambda, t)=\left(k_{r a} f_{a}(\lambda)-k_{r b} f_{b}(\lambda) \frac{k_{a b}}{k_{a}+k_{a b}-k_{b}+k_{r a}-k_{r b}}\right) e^{-\left(k_{a}+k_{a b}+k_{r a}\right) t} \\
+k_{r b} f_{b}(\lambda) \frac{k_{a b}}{k_{a}+k_{a b}-k_{b}+k_{r a}-k_{r b}} e^{-\left(k_{b}+k_{r b}\right) t} \quad \text { eq. } 6
\end{array}
$$

In Eq. 6, the terms with the same rate of decay in the exponential functions were grouped together. Equation 6 can be rewritten as: 


$$
I(\lambda, t)=D A S_{1}(\lambda) \exp \left(-k_{1} t\right)+D A S_{2}(\lambda) \exp \left(-k_{2} t\right) \quad \text { eq. } 7
$$

In this simple example $k_{1}$ and $k_{2}$ are:

$$
\begin{gathered}
k_{1}=\left(k_{a}+k_{a b}+k_{r a}\right) \text { eq. } 8 \\
k_{2}=\left(k_{b}+k_{r b}\right) \quad \text { eq. } 9
\end{gathered}
$$

$D A S_{1}(\lambda)$ and $D A S_{2}(\lambda)$, which are so-called decay associated spectra (DAS), are:

$$
\begin{gathered}
D A S_{1}=\left(k_{r a} f_{a}(\lambda)-k_{r b} f_{b}(\lambda) \frac{k_{a b}}{k_{a}+k_{a b}-k_{b}+k_{r a}-k_{r b}}\right) \quad \text { eq. } 10 \\
D A S_{2}=k_{r b} f_{b}(\lambda) \frac{k_{a b}}{k_{a}+k_{a b}-k_{b}+k_{r a}-k_{r b}} \quad \text { eq. } 11
\end{gathered}
$$

By fitting the experimental data to Eq. 7 we obtain $k_{1}, k_{2}, D A S_{1}(\lambda)$, and $D A S_{2}(\lambda)$. Figure 13A-D shows the plots of $D A S_{1}$ and $D A S_{2}$ for different values of the rates involved in Figure 11 and Table 1 shows the rates used for the plots in Figures 13AD.

In Figures 13A-D, there is one lifetime associated with each DAS. These lifetimes are obtained from the rates presented in eqs. 8-9 (the lifetime is the inverse of the rate). In Figure 13A, three different rates for $k_{a b}$ were used, which resulted in three different pairs of DAS (solid, dotted, and dashed) and the corresponding three different pairs of lifetimes.

In Figure 13A consider only the black solid and dotted DAS which represent DAS 1 and $\mathrm{DAS}_{2}$. DAS 1 reflects EET from pigments $a$ to pigments $b$ (the lifetimes associated with each DAS are presented in the legend). This is indicated by the positive peak at $650 \mathrm{~nm}$ (pigment a emission peak) and the negative peak at $700 \mathrm{~nm}$ (pigment $b$ emission peak). This is easy to see from the analytical expression of DAS $_{1}$. At wavelengths near the emission peak of pigment $a$, the emission of pigment $b$ can be considered zero so that $\mathrm{DAS}_{1}$ becomes $k_{r a} f_{a}(\lambda)$ which is positive. At wavelengths near the emission peak of pigment $b$ we can consider the emission from pigments $a$ to be zero so DAS 1 at these wavelengths becomes $-k_{r b} f_{b}(\lambda) \frac{k_{a b}}{k_{a b}-k_{b}}$. Most of the time the rate of EET is the fastest rate involved so 

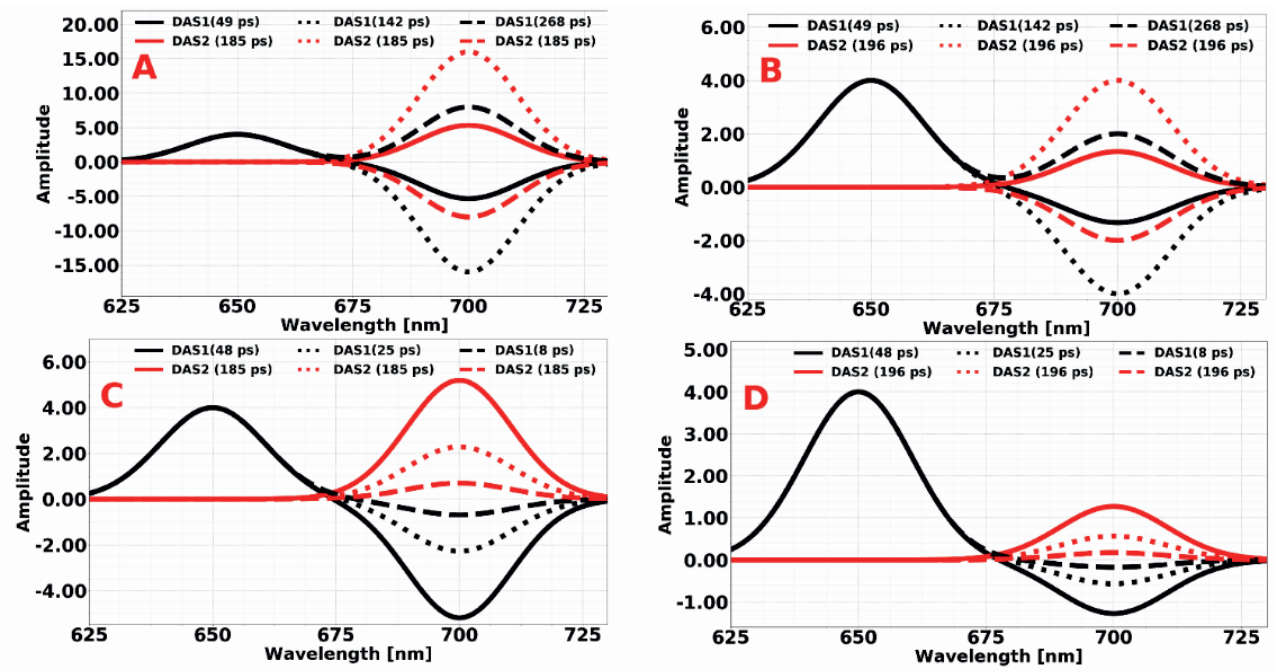

Figure 13. A) DAS ${ }_{1}$ and $D S_{2}$ from Eq. 10-11 are plotted for various values of the EET rate from pigments $a$ to pigments $b$. The radiative rates of both pigments are equal. B) DAS ${ }_{1}$ and DAS $_{2}$ are plotted with the same rates as in $A$ with the exception of the radiative rates. The radiative rate of pigment $a$ is 4 times larger than that of pigment $b . C, D)$ DAS $_{1}$ and DAS $_{2}$ are plotted for different value of the non-radiative rate $\boldsymbol{k}_{a}$. In $\mathrm{C}$ the radiative rates of the both types of pigments are equal and in $D$ the radiative rate of pigments $a$ is 4 times larger than that of pigments $b$. All the rates used are presented in Table 1. Notice that in this simple example the amplitude of DAS ${ }_{1}$ does not change for different values of $\boldsymbol{k}_{a b}$.

$\frac{k_{a b}}{k_{a b}-k_{b}}$ is positive and the DAS becomes negative due to the minus sign. The lifetime associated with $\mathrm{DAS}_{1}$ represents the rate of EET if the radiative and non-radiative decay rate of pigment $a$ are small compared to the rate of EET (see eq. 8).

$\mathrm{DAS}_{2}$ shows the decay of pigment $b$ emission due to transfer of excitation energy to the RCs. This DAS appears with a positive amplitude as long as the rate of EET is larger than the rate of charge separation, which is true in most cases. The lifetime associated with this DAS represents the charge separation time scale if the radiative decay rate and other non-radiative decay rates of pigment $b$ are small compared to the rate of charge separation (see eq. 9). 
Table 1. The rates used in the plots of Figure 13 are shown. Where there are three values for a given process (such as EET or radiative processes) the values belong to the solid, dotted, dashed graphs, respectively.

\begin{tabular}{|c|c|c|c|c|c|}
\hline & $\mathrm{k}_{\mathrm{a}}$ & $\mathrm{k}_{\mathrm{b}}$ & $\mathrm{k}_{\mathrm{ra}}$ & $\mathrm{k}_{\mathrm{rb}}$ & $\mathrm{k}_{\mathrm{ab}}$ \\
\hline$A$ & 0 & $(200 \mathrm{ps})^{-1}$ & $(2500 \mathrm{ps})^{-1}$ & $(2500 \mathrm{ps})^{-1}$ & $\begin{array}{l}(50 \mathrm{ps})^{-1},(150 \mathrm{ps})^{-1}, \\
(300 \mathrm{ps})^{-1}\end{array}$ \\
\hline$B$ & 0 & $(200 \mathrm{ps})^{-1}$ & $(2500 \mathrm{ps})^{-1}$ & $(10000 \text { ps })^{-1}$ & $\begin{array}{l}(50 \mathrm{ps})^{-1},(150 \mathrm{ps})^{-1}, \\
(300 \mathrm{ps})^{-1}\end{array}$ \\
\hline $\mathrm{C}$ & $\begin{array}{ll}(2500 & \text { ps })^{-1}, \\
(150 & \text { ps })^{-1}, \\
(10)^{-1} & \end{array}$ & $(200 \mathrm{ps})^{-1}$ & $(2500 \mathrm{ps})^{-1}$ & $(2500 \mathrm{ps})^{-1}$ & $(50 \mathrm{ps})^{-1}$ \\
\hline $\mathrm{D}$ & $\begin{array}{ll}(2500 & \text { ps })^{-1}, \\
(150 & \text { ps })^{-1}, \\
(10)^{-1} & \end{array}$ & $(200 \mathrm{ps})^{-1}$ & $(2500 \mathrm{ps})^{-1}$ & $(10000 \mathrm{ps})^{-1}$ & $(50 \mathrm{ps})^{-1}$ \\
\hline
\end{tabular}

The dashed DAS in Figure 13A correspond to a different value of $k_{a b}$ which is smaller than the rate of charge separation. When $k_{a b}$ is slower than $k_{b}$ (the dashed DAS 1 and $\mathrm{DAS}_{2}$ ), the negative amplitude of $\mathrm{DAS}_{1}$ becomes positive and the positive amplitude of $\mathrm{DAS}_{2}$ becomes negative. Still the negative amplitude indicates that the pigments with a fluorescence peak at around $700 \mathrm{~nm}$ are receiving excitation energy. However, the rate of EET (or its lifetime) is still the one that is associated with DAS 1.

Figure 13B shows $\mathrm{DAS}_{1}$ and $\mathrm{DAS}_{2}$ with similar parameters as in Figure $13 \mathrm{~A}$ with the difference that the radiative rate of pigments $a$ is 4 times larger than for pigments $b$ in Figure 13B. In the solid black DAS ${ }_{1}$ of Figure $13 \mathrm{~B}$ the emission peak of pigments $a$ is higher than that of pigments $b$. This is because the radiative rate of pigments $a$ is considerably larger than that of pigments $b$. However, we observe the same behavior if the non-radiative rate of pigments $a$ is faster than the non-radiative rate of pigments $b$, see Figure $13 \mathrm{C}$ dotted and dashed DAS 1 .

Figures $13 \mathrm{C}$ and 13D show the effect of a non-zero rate for $k_{a}$ for three different values. The radiative rates of pigments $a$ and $b$ are the same in Figure 13C. The radiative rate of pigments $a$ is 4 times larger than that of pigments $b$ in Figure 13D. Due to the large value of $k_{a}$ in Figures 13C-D for the dotted and dashed graphs, the 26 
lifetime associated with $\mathrm{DAS}_{1}$ does not represent the rate of EET anymore. According to Table 1, the rate of EET is (50 ps) $)^{-1}$, but the corresponding lifetimes of dotted and dashed $\mathrm{DAS}_{1}$ are 25 ps and 8 ps respectively.

If in Eq. 7 we set the time to zero we get the fluorescence at time zero which is the sum of the DAS involved. In our simple example, because at time zero only pigments $a$ are excited, the time zero fluorescence is the same as the emission spectrum of pigments $a$.

Also, if there are more (for instance $N$ ) pigments involved with EET between them, it is always possible to transform the solution of the first order differential equation to the general form:

$$
I(\lambda, t)=\sum_{i=1}^{N} D A S_{i}(\lambda) \times \exp \left(-k_{i} * t\right) \text { eq. } 12
$$

In case of many pigments, the analytical expressions for the DAS and the rates become quite complicated and practically useless. However, the general principles outlined for the two-pigments-only systems still hold, the positive and negative peak in one DAS indicate EET.

\section{Organization of this thesis}

The organization of this thesis is as follows: In Chapter 2, EET in isolated APC trimers was studied, both in crystal and in protein-solution form. Each monomer of APC trimer contains two pigments, a high-energy and a low-energy pigment at a distance of $\sim 51 \AA$. In the trimer, adjacent monomers form three closely spaced ( 21 $\AA$ ) pigment pairs of low- and high-energy. Surprisingly, it was found that $\sim 10 \%$ of these pigment pairs consist of two high-energy pigments instead of the usual pair of low- and high-energy pigments.

In Chapter 3, state-transitions in the cyanobacterium Synechococcus elongatus were studied. We measured $77 \mathrm{~K}$ time-resolved fluorescence of wild-type cells which were either dark-adapted (state II) or illuminated with blue light (state I). Two different excitation wavelengths were used to preferentially excite PBSs or Chls a. By global analysis of the data, the DAS of cells prepared in either state I or state II were 
obtained. The comparison of these DAS in state I and II lead to a new model for state transitions in Synechococcus elongatus.

In Chapter 4, another regulatory process in cyanobacteria was studied. This regulatory process occurs in the cyanobacterium Leptolyngbya ohadii, which is living in the desert where it only performs photosynthesis early in the morning. During this period, both light intensity and temperature are low and because dew is still available, the cells are in a hydrated state. During the day, the cells become desiccated. The high light intensity and lack of water can be very damaging for the photosynthetic apparatus, and therefore severe regulation is required. We performed room temperature and $77 \mathrm{~K}$ time-resolved measurements on hydrated and desiccated cells. The time-resolved data analysis combined with other experimental results lead to a new model for photoprotection in Leptolyngbya ohadii.

In Chapter 5, the EET efficiency of the green sulfur bacterium $C$. tepidum was compared at $77 \mathrm{~K}$ and $293 \mathrm{~K}$. We provided a model for EET between BChl $c$ (chlorosome) and BChl a (baseplate and FMO). Previously, an efficiency of $70 \%$ for trapping in the RCs at $77 \mathrm{~K}$ was reported. Using Global analysis, the efficiency of excitation energy trapping in the RCs was estimated to be $80-90 \%$ and it is also found that this efficiency does not significantly depend on temperature.

Chapter 6 presents the general discussion, in which the results of the chapters twofive are discussed further.

\section{References}

Bína D, Gardian Z, Vácha F, Litvín R (2016) Native FMO-reaction center supercomplex in green sulfur bacteria: an electron microscopy study. Photosynth Res 128:93-102. doi: 10.1007/s11120-015-0205-y

Blankenship RE (2008a) The Basic Principles of Photosynthetic Energy Storage. In: Molecular Mechanisms of Photosynthesis. Wiley-Blackwell, pp 1-10

Blankenship RE (2008b) Electron Transfer Pathways and Components. In: Molecular Mechanisms of Photosynthesis. Wiley-Blackwell, pp 124-156 
Ferreira KN, Iverson TM, Maghlaoui K, et al (2004) Architecture of the Photosynthetic Oxygen-Evolving Center. Science (80- ) 303:1831 LP-1838

Frese R, Oberheide U, van Stokkum I, et al (1997) The organization of bacteriochlorophyll $\mathrm{c}$ in chlorosomes from Chloroflexus aurantiacus and the structural role of carotenoids and protein. Photosynth Res 54:115-126. doi: 10.1023/A:1005903613179

Hauska G, Schoedl T, Remigy H, Tsiotis G (2001a) The reaction center of green sulfur bacteria. Biochim Biophys Acta - Bioenerg 1507:260-277. doi: https://doi.org/10.1016/S0005-2728(01)00200-6

Hauska G, Schoedl T, Remigy H, Tsiotis G (2001b) The reaction center of green sulfur bacteria1Dedicated to the memory of Jan Amesz.1. Biochim Biophys Acta - Bioenerg 1507:260-277. doi: https://doi.org/10.1016/S00052728(01)00200-6

Holzwarth AR, Wendler J, Suter GW (1987) Studies om chromophore coupling in isolated phycobiliproteins. Biophys J 51:1-12. doi: 10.1016/S00063495(87)83306-4

Kirilovsky D (2015) Modulating energy arriving at photochemical reaction centers: Orange carotenoid protein-related photoprotection and state transitions. Photosynth Res 126:3-17. doi: 10.1007/s11120-014-0031-7

Lakowicz JR (2006) Principles of fluorescence spectroscopy

Loefroth JE (1986) Time-resolved emission spectra, decay-associated spectra, and species-associated spectra. J Phys Chem 90:1160-1168. doi:

$10.1021 / \mathrm{j} 100278 \mathrm{a} 040$

MacColl R (2004) Allophycocyanin and energy transfer. Biochim Biophys Acta Bioenerg 1657:73-81. doi: 10.1016/j.bbabio.2004.04.005

Mirkovic T, Ostroumov EE, Anna JM, et al (2016) Light Absorption and Energy Transfer in the Antenna Complexes of Photosynthetic Organisms. Chem Rev acs.chemrev.6b00002. doi: 10.1021/acs.chemrev.6b00002 
Mullineaux CW, Allen JF (1990) State 1-State 2 transitions in the cyanobacterium Synechococcus 6301 are controlled by the redox state of electron carriers between Photosystems I and II. Photosynth Res 23:297-311. doi: 10.1007/BF00034860

Nelson N, Ben-Shem A (2004) The complex architecture of oxygenic photosynthesis. Nat Rev Mol Cell Biol 5:971-982. doi: 10.1038/nrm1525

Nickelsen J, Rengstl B (2013) Photosystem II Assembly: From Cyanobacteria to Plants. Annu Rev Plant Biol 64:609-635. doi: 10.1146/annurev-arplant050312-120124

Novoderezhkin VI, Romero E, Dekker JP, van Grondelle R Multiple ChargeSeparation Pathways in Photosystem II: Modeling of Transient Absorption Kinetics. ChemPhysChem 12:681-688. doi: 10.1002/cphc.201000830

Oostergetel GT, van Amerongen H, Boekema EJ (2010) The chlorosome: a prototype for efficient light harvesting in photosynthesis. Photosynth Res 104:245-255. doi: 10.1007/s11120-010-9533-0

Permentier HP, Schmidt KA, Kobayashi M, et al (2000) Composition and optical properties of reaction centre core complexes from the green sulfur bacteria Prosthecochloris aestuarii and Chlorobium tepidum. Photosynth Res 64:2739. doi: 10.1023/A:1026515027824

Peterman EJ, Dukker FM, van Grondelle R, van Amerongen H (1995) Chlorophyll a and carotenoid triplet states in light-harvesting complex II of higher plants. Biophys J 69:2670-2678. doi: https://doi.org/10.1016/S0006-3495(95)801384

Romero E, van Stokkum IHM, Novoderezhkin VI, et al (2010) Two Different Charge Separation Pathways in Photosystem II. Biochemistry 49:4300-4307. doi: $10.1021 /$ bi 1003926

van Amerongen H, Valkunas L, van Grondelle R (2000) Photosynthetic Excitons. World Scientific

van Oort B, Murali S, Wientjes E, et al (2009) Ultrafast resonance energy transfer 30 
from a site-specifically attached fluorescent chromophore reveals the folding of the N-terminal domain of CP29. Chem Phys 357:113-119. doi:

https://doi.org/10.1016/j.chemphys.2008.10.052

van Stokkum IHM, Larsen DS, van Grondelle R (2004) Global and target analysis of time-resolved spectra. Biochim Biophys Acta - Bioenerg 1657:82-104. doi: http://doi.org/10.1016/j.bbabio.2004.04.011

van Stokkum IHM, van Oort B, van Mourik F, et al (2008) (Sub)-Picosecond Spectral Evolution of Fluorescence Studied with a Synchroscan StreakCamera System and Target Analysis. In: Aartsma TJ, Matysik J (eds) Biophysical Techniques in Photosynthesis. Springer Netherlands, Dordrecht, pp 223-240

Zlenko D V, Galochkina T V, Krasilnikov PM, Stadnichuk IN (2017) Coupled rows of PBS cores and PSII dimers in cyanobacteria: symmetry and structure. Photosynth Res 133:245-260. doi: 10.1007/s11120-017-0362-2 


\section{Picosecond excitation energy transfer of}

\section{allophycocyanin studied in solution and in crystals}

Ranjbar Choubeh, R. ${ }^{*}$, Sonani, R.R. ${ }^{*}$ Madamwar, D. et al. Photosynth Res (2018) 135: 79. https://doi.org/10.1007/s11120-017-0417-4 (*equal contribution) 


\section{ABSTRACT}

Cyanobacteria perform photosynthesis with the use of large light-harvesting antennae called phycobilisomes (PBSs). These hemispherical PBSs contain hundreds of open-chain tetrapyrrole chromophores bound to different peptides, providing an arrangement in which excitation energy is funnelled towards the PBScore from where it can be transferred to photosystem I and/or photosystem II. In the PBS-core many allophycocyanin (APC) trimers are present, red-light absorbing phycobiliproteins that covalently bind phycocyanobilin (PCB) chromophores. APC trimers were amongst the first light-harvesting complexes to be crystallized. APC trimers have two spectrally different PCBs per monomer, a high- and a low-energy pigment. The crystal structure of the APC-trimer reveals the close distance $(\sim 21 \AA)$ between those two chromophores (the distance within one monomer is $\sim 51 \AA$ ) and this explains the ultrafast ( $\sim 1$ ps) excitation energy transfer (EET) between them. Both chromophores adopt a somewhat different structure, which is held responsible for their spectral difference. Here we used spectrally resolved picosecond fluorescence to study EET in these APC trimers both in crystallized and in solubilized form. We found that not all closely spaced pigment couples consist of a low- and a high-energy pigment. In $\sim 10 \%$ of the cases, a couple consists of two high-energy pigments. EET to a low-energy pigment, which can spectrally be resolved, occurs on a time scale of tens of picoseconds. This transfer turns out to be 3 times faster in the crystal than in solution. The spectral characteristics and the time scale of this transfer component are similar to what has been observed in whole cells of Synechocystis sp. PCC 6803, for which, it was ascribed to EET from C-phycocyanin to APC. The present results thus demonstrate that part of this transfer should probably also be ascribed to EET within APC trimers. 
Picosecond excitation energy transfer of allophycocyanin studied in solution and in crystals

\section{INTRODUCTION}

Cyanobacteria harvest light for photosynthesis via huge membrane-bound, multimolecular complexes, the phycobilisomes (PBSs). The PBS is a well-organized stack of coloured phycobiliproteins (PBPs) and colour-less linker peptides. The PBPs bind open-chain tetrapyrrole chromophores, which would otherwise be insoluble in the cellular aqueous environment. During the last two decades, it has been shown that PBPs do not just hold chromophores, but also play a vital role in defining the chromophores' spectral characteristics, which determine the direction, rate and efficiency of excitation energy transfer (EET) (for reviews see (Sidler 1994; Watanabe and Ikeuchi 2013)).

Allophycocyanin (APC) is a red-light absorbing PBP that covalently binds phycocyanobilin (PCB) chromophores. Together with other proteins such as $\mathrm{Lcm}_{\mathrm{c}}$, Lc and allophycocyanin-B, APC forms the core of the PBS (Gingrich et al. 1983; Ducret et al. 1998). APC is a trimer (radius: $\sim 11 \mathrm{~nm}$, thickness: $\sim 3 \mathrm{~nm}$ ) of monomers, each consisting of two peptides, called the $\alpha$ - and $\beta$-subunits, both containing a single PCB attached to a conserved cysteine residue.

Monomeric APC has an absorption spectrum similar to that of C-phycocyanin (CPC). However, when APC monomers form a trimer, the absorption spectrum shows a red-shift of $\sim 30 \mathrm{~nm}$ (Lundell and Glazer 1981; MacColl 2004; McGregor et al. 2008) as compared to, for instance, C-PC trimers. This enables APC to be the energy transfer mediator between higher-energy absorbing peripheral PBPs and lower-energy absorbing $\mathrm{L}_{\mathrm{CM}}$ and/or allophycocyanin-B, which further transfer energy to the reaction centre chlorophylls. Upon trimer formation, the PCB chromophore of the $\alpha$-subunit of one monomer comes close to a PCB, attached to the $\beta$-subunit of another monomer. The close proximity of these chromophores is accompanied by a red-shift, although the physical nature of this red-shift is still under debate and various mechanisms have been proposed. (Peng et al. 2014) suggested that the unique geometry of $\mathrm{PCB}$ in APC causes the red-shift. (McGregor et al. 2008) proposed that the red-shift is due to special coupling of the hydrophobic protein micro-environment around $\mathrm{APCB}$ created by the $\beta$-subunit. 
Excitonic interaction between the $\alpha \mathrm{PCB}$ and $\beta \mathrm{PCB}$ in adjacent monomers has also been proposed to cause the red-shift (MacColl 2004).

Based on the existing literature it was concluded in (van Amerongen $\mathrm{H}$, Valkunas L, van Grondelle R 2000) that EET between both pigments occurs via incoherent downhill Förster transfer from the pigment in the $\beta$-subunit to the one in the $\square$ subunit with a time constant in the order of 1 ps. Further equilibration between these pigment pairs within the trimer on the other hand takes tens of ps but it is not expected to be accompanied by significant spectral changes. However, in some recent experiments on APC crystals, we observed some large unexpected spectral changes on a time scale of tens of picoseconds. To study this apparent controversy in more detail, we performed time-resolved fluorescence measurements on APC trimers, purified from Phormidium sp. A9DM, in crystalline and solution form (Sonani et al. 2015).

\section{Materials \& Methods}

\subsection{Protein preparation and crystallization}

The APC 660 (referring to the fluorescence maximum at $660 \mathrm{~nm}$ ) trimers were purified from Phormidium sp. A9DM and crystallized as described earlier (Sonani et al. 2015). The crystals did not show any sign of APC 680 fluorescence.

\subsection{Steady-state absorption measurements}

The steady-state absorption spectrum of APC in $0.05 \mathrm{M}$ phosphate buffer ( $\mathrm{pH} 8.0)$ was recorded at $25 \pm 0.2{ }^{\circ} \mathrm{C}$ on a UV-visible spectrophotometer (Specord 210, Analytik Jena AG, Jena, Germany).

\subsection{Fluorescence lifetime imaging microscopy (FLIM)}

Time-correlated single-photon-counting FLIM measurements on crystals were performed similarly as in earlier studies in our laboratory on crystals of other photosynthetic complexes (Pascal et al. 2005; van Oort et al. 2008, 2011, 2014). The measurements were done on a Leica SP5X-SMD multi-mode confocal laser- 
Picosecond excitation energy transfer of allophycocyanin studied in solution and in crystals

scanning microscope using a 63x water immersion 1.2 NA lens. APC crystals were excited using a white-light laser (WLL or super continuum laser), which emits a continuous spectrum between 470 and $670 \mathrm{~nm}$, from which individual excitation wavelengths can be selected. Confocal imaging was performed using internal filterfree spectral hybrid detectors. Excitation was performed at $594 \mathrm{~nm}$ and fluorescence was detected around $645 \mathrm{~nm}$ with a spectral bandwidth of 5-10 nm. Detection was not performed in the fluorescence maximum in order to avoid saturation of the detector. FLIM images with a frame size of $128 \times 128$ pixels were acquired with an SPC730 TCSPC imaging module (Becker \& Hickl, Berlin, Germany). The images were analysed with the SPCImage software (Becker \& Hickl, version 3.2.3.0, Berlin, Germany).

Each pixel contains a time trace of the fluorescence emission up to several nanoseconds. The size or scale of the image is $246 \mu \mathrm{m} \times 246 \mu \mathrm{m}$. The timeresolved fluorescence of each pixel of the FLIM image was fitted with a single exponential decay:

$a \times \exp \left(\frac{-t}{\tau}\right)$

where $a$ is the amplitude, $\tau$ is the fluorescence lifetime, and $t$ is the time after excitation. Each pixel in the FLIM image is colour-coded, using SPClmage, according to the fluorescence lifetime of that pixel. The lifetimes are also presented in a histogram in which the horizontal axis represents the lifetime in picoseconds and the vertical axis the corresponding pixel frequency of these lifetimes. Only those pixels were selected for which the peak was at least $40 \%$ as high as the brightest pixel in the image in order to obtain a good signal to noise ratio. A typical maximum peak value was $\sim 700$ photons. The lifetimes were also calculated after binning each pixel with the 24 surrounding pixels (average of in total $5 \times 5$ pixels).

\subsection{Time-resolved fluorescence spectroscopy}

Time-resolved fluorescence measurements were performed with a picosecond streak camera at room temperature (van Stokkum et al. 2008; Tian et al. 2011; 
Chukhutsina et al. 2015) and a laser repetition rate of $4 \mathrm{MHz}$. The APC crystals and protein solution were put on thin glass plates and were excited using a Nikon CFI Plan Apo Lambda 10x objective lens with an excitation wavelength of $590 \mathrm{~nm}$ and a power of 3-7 nW. The excitation of APC crystals was achieved in the following way. The sample was scanned with the focused laser beam and the sudden detection of an intense fluorescence signal that would disappear again by a slight movement of the excitation light demonstrated that an APC crystal was being excited.

The images were corrected for the wavelength dependency of the detector and then sliced into $5 \mathrm{~nm}$ wide time traces. The fluorescence kinetics of APC crystals were recorded with a 800 ps and 2000 ps time window and those of APC proteins in solution with a time window of 2000 ps.

Global and target analysis were performed as described in (Tian et al. 2012; Chukhutsina et al. 2015), using Glotaran (Snellenburg et al. 2012) and the TIMP (Mullen and van Stokkum 2007) package for R.

The average fluorescence lifetime for different detection wavelengths was calculated according to:

$\frac{\sum_{i} \tau_{i} \times D A S_{i}(\lambda)}{\sum_{i} D A S_{i}(\lambda)}$

where $\lambda$ is the wavelength of detection, $D A S_{i}(\lambda)$ is the $i^{\text {th }}$ decay associated spectrum (DAS) obtained from global analysis and $\tau_{i}$ is the corresponding lifetime. The time-zero spectrum was obtained by adding all the DAS that were obtained from the global analysis for one sample. This sum spectrum represents the fluorescence spectrum directly after excitation and relaxation to the $Q_{y}$ states if no additional fast relaxation processes occur that are not captured by the fitting procedure. All the DAS presented in this work are normalized to the maximum of the corresponding time-zero spectrum unless stated otherwise. 
Picosecond excitation energy transfer of allophycocyanin studied in solution and in crystals

\section{Results and Discussion}

\subsection{Steady-state absorption spectrum and chromophore conformation}

In Fig. 1 the absorption spectrum of APC is given together with its Gaussian decomposition, showing four separate components (black dashed lines, 1-4). The absorption spectrum shows a sharp absorption band at $653 \mathrm{~nm}$ with a broad shoulder band centred at $620 \mathrm{~nm}$. APC contains two PCBs: one in the a-subunit bound to a81Cys and another in the $\beta$-subunit bound to $\beta 81 C y$ (hereafter $\alpha \mathrm{PCB}$ and $\beta$ PCB, respectively) ((Sonani et al. 2015), PDBID: 4RMP). It is well established that the APC phycocyanobilins with a high deviation from co-planarity of their four pyrrole rings absorb light at shorter wavelengths than PCBs with a low deviation (Peng et al. 2014). PCB contains four pyrrole rings designated as A, B, C and D, and it is connected to the protein via its A ring (Fig. S1a). In Phormidium APC, the deviation from co-planarity of the rings in $\beta P C B$ is higher than that in aPCB (Figs. S1b-c) ((Sonani et al. 2015), PDB ID: 4RMP). Therefore, it is most likely that the Gaussian absorption components 1 and 2 are due to $\beta$ PCB whereas the other two (3 and 4) should be contributed by $\mathrm{APCB}$, which is in agreement with earlier assignments (see e.g. (van Amerongen H, Valkunas L, van Grondelle R 2000)). The two chromophores in an $\alpha \beta$-monomer are separated by more than $50 \AA$; however, the aPCB of one monomer is only $21 \AA$ away from the $\beta P C B$ of the adjacent monomer within the APC trimer. APC trimers contain three such pairs of spatially clustered low (aPCB) (absorption peak at $\sim 630 \mathrm{~nm}$ and $\sim 653 \mathrm{~nm}$ ) and high ( $\beta P C B$ ) (absorption peak at $\sim 575 \mathrm{~nm}$ and $\sim 620 \mathrm{~nm}$ ) -deviation (from coplanarity) chromophores and all $\alpha \mathrm{PCBs}$ are arranged more towards the periphery of the trimer ring as compared to $\beta P C B$ (Fig. S2). 


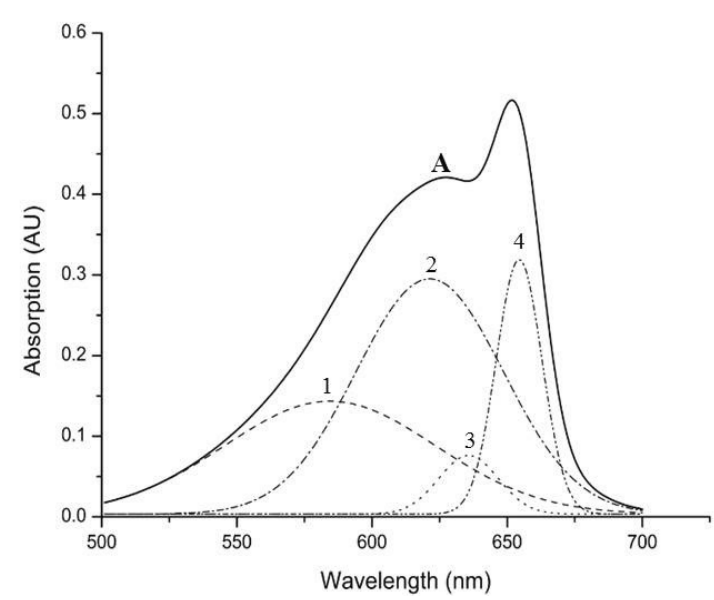

Fig. 1 Absorption spectrum (A, intact line) of purified Phormidium APC. Gaussian decomposition components of the APC absorption spectrum are represented by the dashed lines (1-4)

\subsection{FLIM}

We studied the fluorescence kinetics of APC trimers (in crystals) by FLIM and timeand spectrally-resolved streak-camera measurements. The results of the FLIM measurements on APC crystals are presented in Fig. 2. The crystals were excited at $594 \mathrm{~nm}$ and the fluorescence was recorded at $645 \mathrm{~nm}$. One lifetime was enough to fit the kinetics per pixel. The fitted fluorescence lifetime for the crystals mainly ranged from 650 ps to 700 ps and the crystals showed a homogeneous lifetime distribution (see the histogram in Fig. 2). The fluorescence lifetime for different detection wavelengths was also calculated using the global analysis of streakcamera images and the results are presented in Fig. S3. 

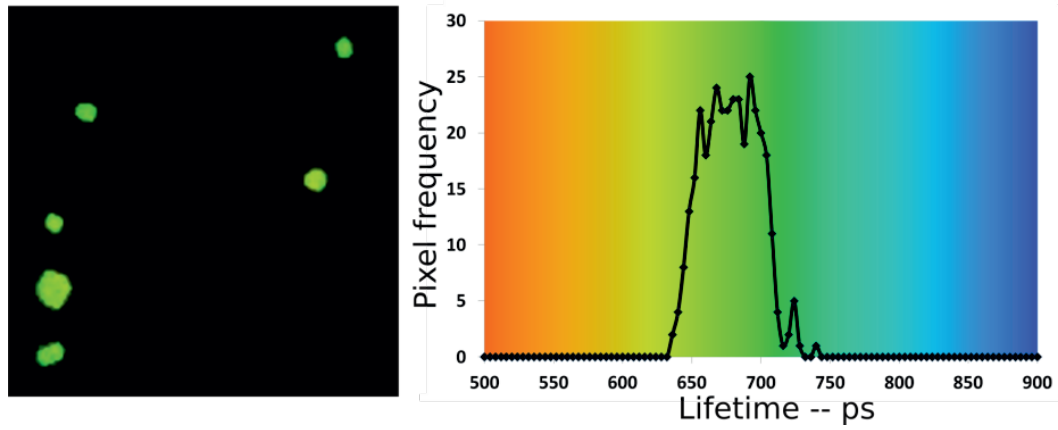

Fig. 2 FLIM image of APC crystals (left) and the corresponding histogram of the lifetimes (right). The FLIM image is made up of $128 \times 128$ pixels. The image of the crystals roughly contains $\sim 400$ pixels. The size of the FLIM image is $246 \mu \mathrm{m} \times 246 \mu \mathrm{m}$. The excitation and detection wavelengths are $594 \mathrm{~nm}$ and $645 \mathrm{~nm}$

\subsection{Time-resolved fluorescence measurements on APC crystals and APC protein solution}

APC crystals and the APC protein solution were subsequently measured with the streak-camera setup and the data were globally analysed (see Materials and Methods). In all cases, three lifetimes were required to fit the data, in contrast to the FLIM data, where the signal to noise ratio was much lower and the timeresolution was less. The fitting results of APC crystals and of APC protein solution are presented in Figs. 3a-b. Figs. 3c-d show selected measured and fitted time traces of APC crystals and APC protein solution, respectively. Each DAS is scaled according to the maximum of the estimated time-zero spectrum. The 25 ps DAS for the crystals and the 67 ps DAS for the protein solution have very similar characteristics, showing a broad positive band below $650 \mathrm{~nm}$ and a negative band peaking between 660 and $665 \mathrm{~nm}$. Based on the spectral differences between $\beta P C B$ and $\alpha \mathrm{PCB}$ described above, we assign the 25 ps and 67 ps DAS in Fig. 3 to EET from $\beta P C B$ to aPCB. Also in (Holzwarth et al. 1990) using time-resolved fluorescence spectroscopy the authors obtained a 25 ps DAS with a positive peak at $650 \mathrm{~nm}$ and a broad negative peak at 660-690 nm. The broadness of the negative peak was explained by a broad range of conformations due to a lack of linker peptides. Time-resolved measurements on wild-type (WT) Synechocystis sp. PCC 6803 revealed a 43 ps DAS with a similar shape (see SI and Fig. S4a). 
In Fig. 3 the 90 ps DAS of the crystals and the 445 ps DAS of the APC protein solution showed positive maxima at $660 \mathrm{~nm}$ and 660-665 nm, respectively. These positive DAS represent only decay of fluorescence. In (Holzwarth et al. 1990) a 720 ps DAS was observed with a broad positive peak centred at $660 \mathrm{~nm}$ together with a third DAS with a lifetime of $1700 \mathrm{ps}$, also centred at $660 \mathrm{~nm}$. The presence of such a broad band together with the presence of different long lifetimes was ascribed to conformational heterogeneity. However, the 90 ps DAS we obtained has a narrow shape that suggests a well-defined conformation of the corresponding chromophores.

In Fig. 3 the 820 ps and 1920 ps DAS of crystals and protein solution, respectively, showed a positive peak at 660-665 nm, which reflects fluorescence decay. Both DAS reflect a well-defined conformation and have the same shape (Fig. S4c).

In the literature, it is generally reported that EET between spectrally different chromophores in APC protein solution occurs with a time constant of $\sim 1$ ps or less (van Amerongen H, Valkunas L, van Grondelle R 2000). Although slow processes were reported before, they were not assigned to EET; for example as discussed earlier, the 25 ps DAS obtained in (Holzwarth et al. 1990) was assigned to the decay from the upper excitonic level to the lower excitonic level. In (Zhang et al. 1998) both time-resolved isotropic and anisotropic fluorescence spectroscopy revealed, amongst other components, a 40 ps component. The authors assigned the 40 ps lifetime to EET between $\beta 84$ PCB chromophores in the centre of the APC trimer after exciton localization had occurred within several picoseconds. However, it is not clear how the $\sim 40$ ps lifetime could be observed in the isotropic measurements. In (Beck and Sauer 1992), one-colour pump-probe experiments resolved, amongst other components, a 45 \pm 10 ps decay component between $\sim 590$ $\mathrm{nm}$ and $\sim 650 \mathrm{~nm}$, with positive amplitude and no negative amplitude at longer wavelengths. The high power of $5-8 \mathrm{~mW}$ that was used, potentially leading to singlet-singlet annihilation, could have been the reason for the absence of a rise term (a negative amplitude) at longer wavelengths. 
a)

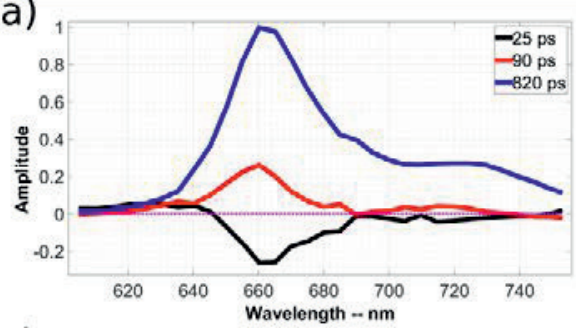

c)

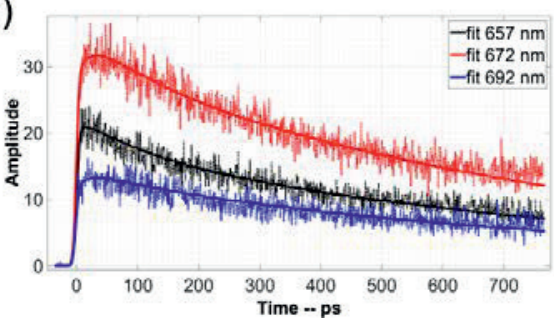

b)

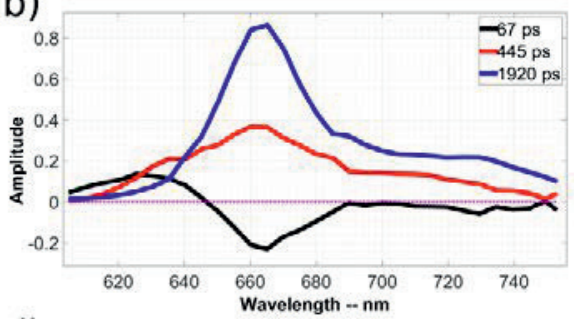

d)

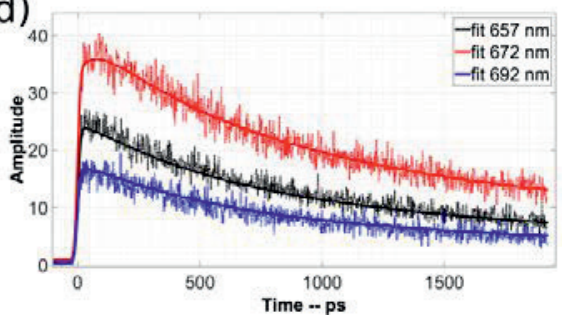

Fig. 3 a-b) DAS obtained from the global analysis of fluorescence data of APC crystals and APC protein solution as measured with the streak camera are shown in a and $b$, respectively. The excitation wavelength was $590 \mathrm{~nm}$. The DAS were normalized to the maximum of the time-zero spectrum. c-d) Selected measured and fitted time traces of APC crystals and APC protein solution. The numbers in the legends indicate the detection wavelength. The solid lines represent the fits to the time traces 


\subsection{Target analysis}

To estimate the various spectral components and their contribution to the overall kinetics, we have performed target analysis. Because we resolved three DAS we also used three compartments. At least two of these should have a different spectrum because the fastest DAS reflects EET between components with different spectra. The target models for crystals and APC protein solution are shown in Figs. 4a-b. The initial fractional populations, which provided the best fits, are given for each compartment.

As we stated before both ultrafast and slow EET processes occur in APC trimers. This is justified by the presence of ultrafast EET as reported in the literature and the tens of ps EET process in the present work but also reported in for instance (Holzwarth et al. 1990). The monomeric unit of APC trimers contains aPCB and $\beta P C B$. Regardless which of the $\mathrm{APCB}$ and $\beta \mathrm{PCB}$ has the lower energy, we call them red and blue spectral forms so that the red form represents the lower energy species. According to the absorption decomposition of Fig. 1 initially only the blue forms are excited. If there was only slow EET, then the initial population of the blue form would have been 1, and if there was only ultrafast EET the initial population of the blue form would have been 0 and we would not have been able to observe EET in our measurements as the time resolution of our setup was $\sim 10$ ps. The initial population that gives the best fit shows that at time zero the majority of the population resides already in the red spectral form and to a lesser extent in the blue spectral from. This confirms that ultrafast and slow EET co-exist.

Considering the crystal structure, the coexistence of "fast" and "slow" downhill EET is unexpected. We therefore propose that not all the closely spaced $\alpha$-and $\beta P C B s$, dashed-orange ovals in Fig. S2, contain a red and blue spectral form, but some of them contain a blue-blue pair. Only after the initial ultrafast EET between red and blue forms has taken place, there is excitation energy transfer from a blue-blue pair to the red form in a neighbouring red-blue pair. Using the obtained initial populations, the percentage of these blue-blue pairs can be estimated. If the ultrafast EET happens only between the blue and red forms, then after $\sim 1$ ps the only population remaining in the blue spectral form consists of blue-blue pairs. The 
Picosecond excitation energy transfer of allophycocyanin studied in solution and in crystals

time resolution of our measurements as stated before is $\sim 10$ ps so we cannot resolve the initial ultrafast EET and the slow EET occurs between the blue-blue and red-blue forms after the ultrafast EET has already occurred. If there are $n$ blueblue pairs and $m$ red-blue pairs then the ratio of the number of blue spectral forms in the blue-blue pairs to the total number of blue spectral forms is $\frac{2 n}{2 n+m}$. This ratio should be the same as the initial population of the blue-blue pairs, which is 0.2 in Fig. 4. This results in $m=8 n$, meaning that $\sim 10 \%$ of the closely spaced $\alpha-$ and $\beta$ PCBs are blue-blue pairs and $\sim 90 \%$ are red-blue pairs.

In Figs. 4a-b the ratio of the rate of EET from the blue to the red compartment to the rate of EET from the blue to the black compartment was made equal to the ratio of the initial populations of the red and black compartments. For example, in Fig. $4 a$ the initial population of the red compartment is 4 times larger than that of the black compartment, so the rate of EET from the blue compartment to the red compartment is 4 times faster than the rate of EET from the blue compartment to the black compartment.

In Fig. 4a the blue compartment transfers its excitation energy to the black and red compartments with rates of $(130 \mathrm{ps})^{-1}$ and $(33 \mathrm{ps})^{-1}$, respectively; as a result the blue compartment decays with a rate of $(26 \mathrm{ps})^{-1}$. The black and red compartments in turn decay to the ground state with rates of $(88 \mathrm{ps})^{-1}$ and $(820 \mathrm{ps})^{-1}$, respectively.

In Fig. 4b the blue compartment transfers its excitation energy to the black and red compartments with rates of $(230 \mathrm{ps})^{-1}$ and $(95 \mathrm{ps})^{-1}$, respectively. The blue compartment then decays with an overall rate of $(67 \mathrm{ps})^{-1}$. The black and red compartments in turn decay to the ground state with rates of (444 ps) $)^{-1}$ and (1920 ps) $)^{-1}$, respectively.

The area under the SAS is proportional to the radiative rate of the corresponding species multiplied by an instrument-dependent factor (Loefroth 1986; Holzwarth et al. 1987). In Fig. 4a the area under the red SAS is $\sim 1.6$ times larger than that of the black SAS. One would then expect that the red SAS decays with a faster radiative rate than the black SAS. However, it is the black SAS that decays $\sim 9$ times faster. This indicates that the origin of the short lifetime of the black SAS must be non- 
radiative in nature. The same argument applies to the SAS in Fig. $4 \mathrm{~b}$, the black SAS has a $\sim 1.33$ larger area than the red SAS, however, it decays $\sim 4.3$ times faster. This indicates that the origin of the shorter lifetime of the back SAS is also non-radiative.

At the moment we can only speculate why not all blue forms are in close contact with a red form. One possible reason is that not all pigment-binding sites are occupied by pigments, which prevents fast EET between closely coupled pigments and only transfer to more distant pigments can occur on a time scale of tens of picoseconds. Although we cannot completely rule out this possibility, it seems more likely that the same bilin molecules can adopt both a blue and a red conformation in the APC trimers. As was already discussed above, a switch from blue to red occurs upon the oligomerization of monomers into trimers and may be linked to a change in planarity of the bilin molecules. Possibly an equilibrium exists between such blue and red forms as was also observed for chlorophyll a molecules in plant light-harvesting complexes (Passarini et al. 2010; Krüger et al. 2011). It was demonstrated with the use of single-molecule fluorescence experiments that the fluorescence maximum of LHCII, the major light-harvesting complex of plants, can switch from a form that has a fluorescence maximum around $675 \mathrm{~nm}$ to a form with a maximum far above $700 \mathrm{~nm}$ on a time scale of seconds to tens of seconds (Krüger et al. 2011). Alternatively, the presence of a dynamic equilibrium between the monomeric and trimeric form may lead to the observed heterogeneity although this seems less likely in the case of crystallized APC.

In conclusion, we have measured excitation energy transfer in APC when present in trimeric form in solution and when crystallized. Most of the EET between blue and red spectral forms occurs within several picoseconds as expected from the crystal structure but there is also $\sim 10 \%$ of the excitations that is transferred on a time scale of tens of picoseconds, meaning that not all supposedly red pigments have adopted a "red conformation". The rate of EET between the blue and red forms differs for solution and crystal and apparently, the crystal form is not exactly the same as the solution form. This might also explain why the fluorescence lifetimes are not identical. The "slow" blue-to-red EET component is also observed 
in whole cells of WT Synechocystis sp. PCC 6803. This component was previously ascribed to EET from C-PC to APC but according to the present results it might also be partly due to EET within APC.
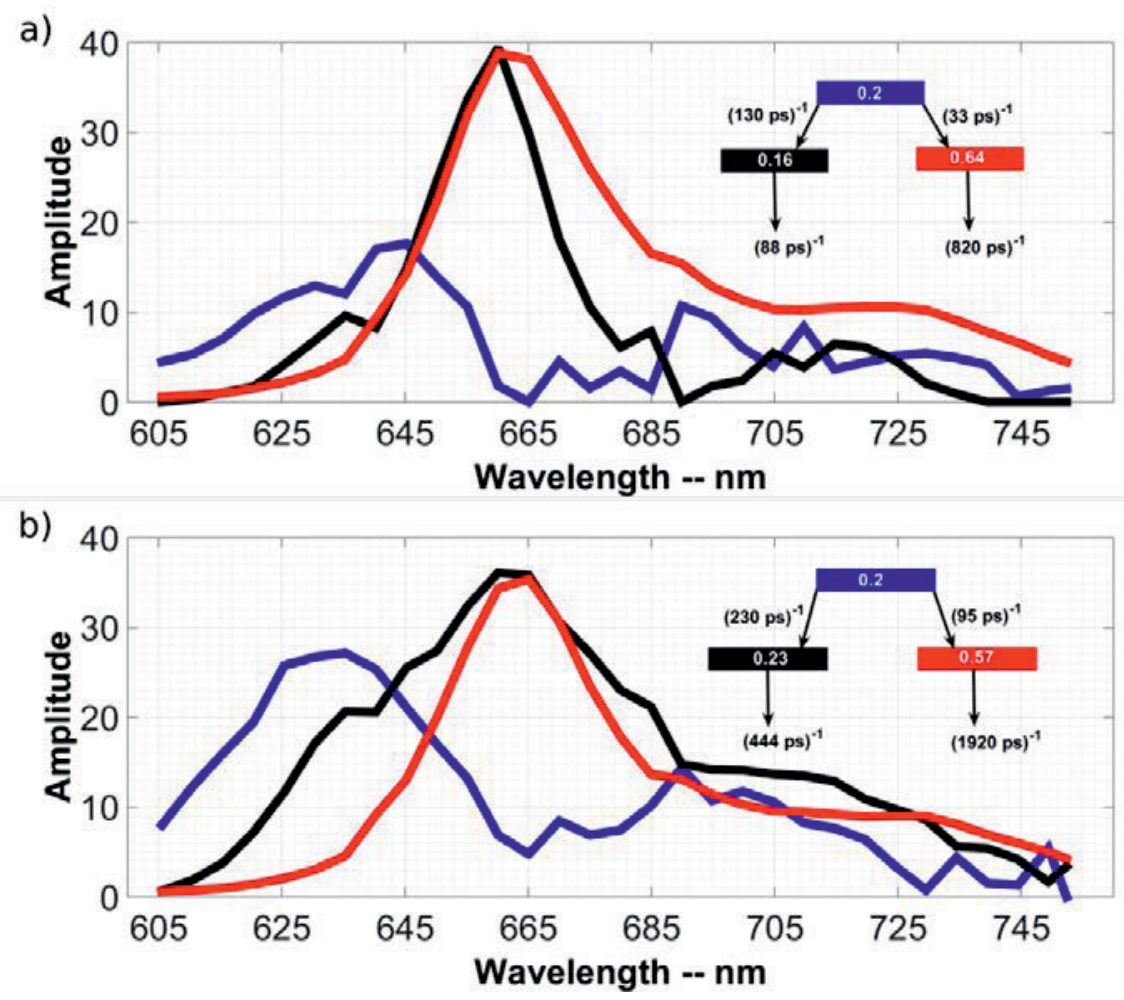

Fig. 4 Three species associated spectra (SAS) as obtained from target analysis are shown for APC crystals and protein solutions in $a$ and $b$, respectively. The model used is shown in the figure inset. Each compartment represents an emitting species and the number written on it is the initial fractional population of excitation. The total initial population is summed to 1 . The colour of each SAS corresponds to the colour of each compartment

\section{Acknowledgements}

The Synechocystis cells used in this study were a kind gift from Dr. D. Kirilovsky. This work is funded by the Foundation for Fundamental Research on Matter (FOM), which is part of The Netherlands Organization for Scientific Research (NWO) (project number 10TBSC24-3). This project was carried out within the research programme BioSolar Cells, co-financed by the Dutch Ministry of 
Economic Affairs. DM acknowledges University Grants Commissions (UGC), New Delhi for BSR Faculty Grant.

\section{Reference List}

Beck WF, Sauer K (1992) Energy-transfer and exciton-state relaxation processes in allophycocyanin. J Phys Chem 96:4658-4666. doi: 10.1021/j100190a094

Chukhutsina V, Bersanini L, Aro E-M, van Amerongen H (2015) Cyanobacterial Light-Harvesting Phycobilisomes Uncouple From Photosystem I During DarkTo-Light Transitions. Sci Rep 5:14193. doi: 10.1038/srep14193

Ducret A, Müller SA, Goldie KN, et al (1998) Reconstitution, characterisation and mass analysis of the pentacylindrical allophycocyanin core complex from the cyanobacterium Anabaena sp. PCC 7120. J Mol Biol 278:369-388. doi: http://dx.doi.org/10.1006/jmbi.1998.1678

Gingrich JC, Lundell DJ, Glazer AN (1983) Core substructure in cyanobacterial phycobilisomes. J Cell Biochem 22:1-14. doi: 10.1002/jcb.240220102

Holzwarth AR, Bittersmann E, Reuter W, Wehrmeyer W (1990) Studies on chromophore coupling in isolated phycobiliproteins: III. Picosecond excited state kinetics and time-resolved fluorescence spectra of different allophycocyanins from Mastigocladus laminosus. Biophys J 57:133-45. doi: 10.1016/S0006-3495(90)82514-5

Holzwarth AR, Wendler J, Suter GW (1987) Studies on Chromophore Coupling in Isolated Phycobiliproteins: II. Picosecond Energy Transfer Kinetics and TimeResolved Fluorescence Spectra of C-Phycocyanin from Synechococcus 6301 as a Function of the Aggregation State. Biophys $J$ 51:1-12. doi: http://dx.doi.org/10.1016/S0006-3495(87)83306-4

Krüger TPJ, Wientjes E, Croce R, van Grondelle R (2011) Conformational switching explains the intrinsic multifunctionality of plant light-harvesting complexes. Proc Natl Acad Sci 108:13516-13521. doi: 10.1073/pnas.1105411108 
Picosecond excitation energy transfer of allophycocyanin studied in solution and in crystals

Loefroth JE (1986) Time-resolved emission spectra, decay-associated spectra, and species-associated spectra. J Phys Chem 90:1160-1168. doi: $10.1021 / \mathrm{j} 100278 \mathrm{a} 040$

Lundell DJ, Glazer AN (1981) Allophycocyanin B. A common beta subunit in Synechococcus allophycocyanin B (lambda max $670 \mathrm{~nm}$ ) and allophycocyanin (lambda max 650 nM). J Biol Chem 256:12600-12606.

MacColl R (2004) Allophycocyanin and energy transfer. Biochim Biophys Acta Bioenerg 1657:73-81. doi: 10.1016/j.bbabio.2004.04.005

McGregor A, Klartag M, David L, Adir N (2008) Allophycocyanin Trimer Stability and Functionality Are Primarily Due to Polar Enhanced Hydrophobicity of the Phycocyanobilin Binding Pocket. J Mol Biol 384:406-421. doi: 10.1016/j.jmb.2008.09.018

Mullen KM, van Stokkum IHM (2007) TIMP: An R Package for Modeling Multi-way Spectroscopic Measurements. J Stat Softw 18:200-6. doi: 10.1359/JBMR.0301229

Pascal AA, Liu Z, Broess K, et al (2005) Molecular basis of photoprotection and control of photosynthetic light-harvesting. Nature 436:134-137. doi: 10.1038/nature03795

Passarini F, Wientjes E, van Amerongen H, Croce R (2010) Photosystem I lightharvesting complex Lhca4 adopts multiple conformations: Red forms and excited-state quenching are mutually exclusive. Biochim Biophys Acta Bioenerg 1797:501-508. doi: https://doi.org/10.1016/j.bbabio.2010.01.015

Peng PP, Dong LL, Sun YF, et al (2014) The structure of allophycocyanin B from Synechocystis PCC 6803 reveals the structural basis for the extreme redshift of the terminal emitter in phycobilisomes. Acta Crystallogr Sect D Biol Crystallogr 70:2558-2569. doi: 10.1107/S1399004714015776

Sidler WA (1994) Phycobilisome and Phycobiliprotein Structures. In: Bryant DA (ed) The Molecular Biology of Cyanobacteria. Springer Netherlands, 
Dordrecht, pp 139-216

Snellenburg JJ, Laptenok SP, Seger R, et al (2012) Glotaran: A Java -Based Graphical User Interface for the R Package TIMP. J Stat Softw 49: doi: 10.18637/jss.v049.i03

Sonani RR, Gupta GD, Madamwar D, Kumar V (2015) Crystal Structure of Allophycocyanin from Marine Cyanobacterium Phormidium sp. A09DM. PLoS One 10:1-16. doi: 10.1371/journal.pone.0124580

Tian L, Gwizdala M, van Stokkum IHM, et al (2012) Picosecond Kinetics of Light Harvesting and Photoprotective Quenching in Wild-Type and Mutant Phycobilisomes Isolated from the Cyanobacterium Synechocystis PCC 6803. Biophys J 102:1692-1700. doi: http://dx.doi.org/10.1016/j.bpj.2012.03.008

Tian L, Van Stokkum IHM, Koehorst RBM, et al (2011) Site, rate, and mechanism of photoprotective quenching in cyanobacteria. J Am Chem Soc 133:1830418311. doi: 10.1021/ja206414m

van Amerongen H, Valkunas L, van Grondelle R (2000) Photosynthetic Excitons. World Scientific

van Oort B, Amunts A, Borst JW, et al (2008) Picosecond fluorescence of intact and dissolved PSI-LHCl crystals. Biophys J 95:5851-61. doi: 10.1529/biophysj.108.140467

van Oort B, Kargul J, Barber J, van Amerongen H (2014) Fluorescence kinetics of PSIl crystals containing $\mathrm{Ca} 2+$ or $\mathrm{Sr} 2+$ in the oxygen evolving complex. Biochim Biophys Acta - Bioenerg 1837:264-269. doi: http://dx.doi.org/10.1016/j.bbabio.2013.11.008

van Oort B, Marechal A, Ruban A V, et al (2011) Different crystal morphologies lead to slightly different conformations of light-harvesting complex II as monitored by variations of the intrinsic fluorescence lifetime. Phys Chem Chem Phys 13:12614-12622. doi: 10.1039/C1CP20331B 
Picosecond excitation energy transfer of allophycocyanin studied in solution and in crystals

van Stokkum IHM, van Oort B, van Mourik F, et al (2008) (Sub)-Picosecond Spectral Evolution of Fluorescence Studied with a Synchroscan StreakCamera System and Target Analysis. In: Aartsma TJ, Matysik J (eds) Biophysical Techniques in Photosynthesis. Springer Netherlands, Dordrecht, pp 223-240

Watanabe M, Ikeuchi M (2013) Phycobilisome: Architecture of a light-harvesting supercomplex. Photosynth Res 116:265-276. doi: 10.1007/s11120-0139905-3

Zhang JJ, Zheng X, Zhang JJ, et al (1998) Studies of the Energy Transfer among Allophycocyanin from Phycobilisomes of Polysiphonia urceolata by TimeResolved Fluorescence Isotropic and Anisotropic Spectroscopy. Photochem Photobiol 68:777-784. doi: 10.1111/j.1751-1097.1998.tb05284.x 


\section{Supplementary Information}

\section{Methods}

Wild-type Synechocystis sp. PCC 6803 cells were measured in a quartz cuvette with an optical length of $1 \mathrm{~cm}$. The OD of the cells was $\sim 0.1$ at $670 \mathrm{~nm}$ in a cuvette of $1 \mathrm{~cm}$ optical length. The cells were excited using $577-\mathrm{nm}$ laser pulses, FWHM $10 \mathrm{~nm}$, and a lens with focal length of $7 \mathrm{~cm}$. The repetition rate of the laser was 4 $\mathrm{MHz}$ and the power of the excitation light was $\sim 7 \mu \mathrm{W}$. The measurements were performed at room temperature.

The images, recorded by the streak camera with a 800 ps time window, were corrected for the wavelength dependency of the detector and then sliced into 2.5-5 $\mathrm{nm}$ wide time traces. Global analysis was performed as described in the main text.

The chemical structure of phycocyanobilin and spatial structure of allophycocyanin trimers

a)

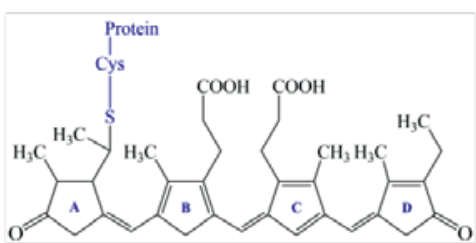

b)

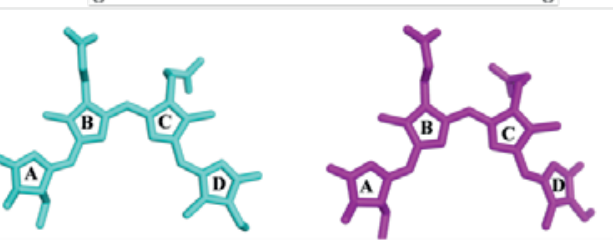

c)
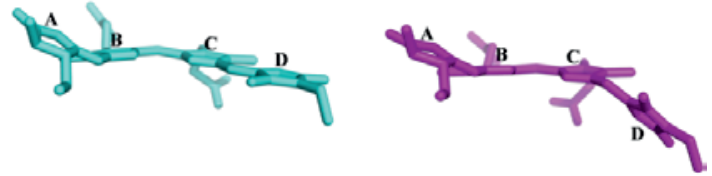

Fig. S1 a) Schematic representation of the PCB chemical structure. b) top view and c) side view of 3D conformation of $\alpha$ PCB (cyan) and $\beta$ PCB (violate) in the Phormidium APC structure (protein data bank code 4RMP). The deviation from co-planarity among the pyrrole rings is notably higher in $\beta P C B$ than in $\alpha P C B$ 


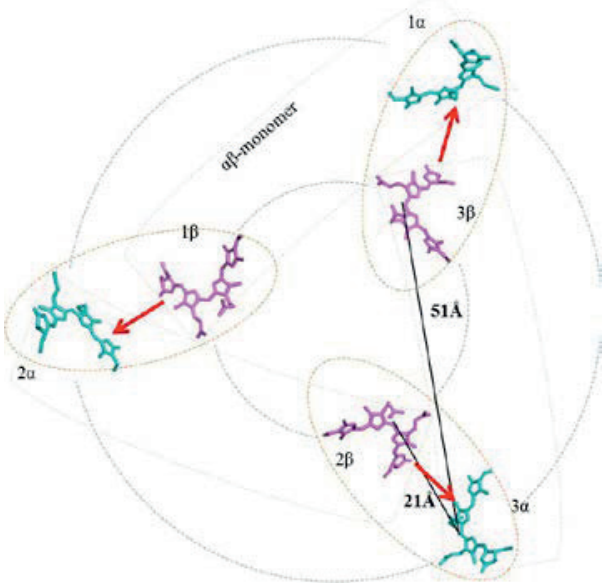

Fig. S2 Spatial arrangement of PCBs in an APC trimer. The aPCBs and $\beta P C B$ s are shown in cyan and violet, respectively. The dashed-grey shape is a schematic representation of the $\alpha \beta$ monomer. The dashed-orange ovals represent the closely spaced ( 21 A centre-to-centre distance) pigments

\section{Average lifetimes calculated by global analysis of streak camera data}

The fluorescence lifetimes for the APC crystals and protein solution detected with streak camera at $645 \mathrm{~nm}$ were calculated as $645 \mathrm{ps}$ and $1.2 \mathrm{~ns}$, respectively (Fig. S3). The fluorescence lifetime of APC crystals detected with the streak camera and with the FLIM setup (detection at $645 \mathrm{~nm}$, see the histogram in Fig. 2) were very similar, which confirms that the time-resolved measurements carried out with the streak camera were indeed performed on APC crystals.

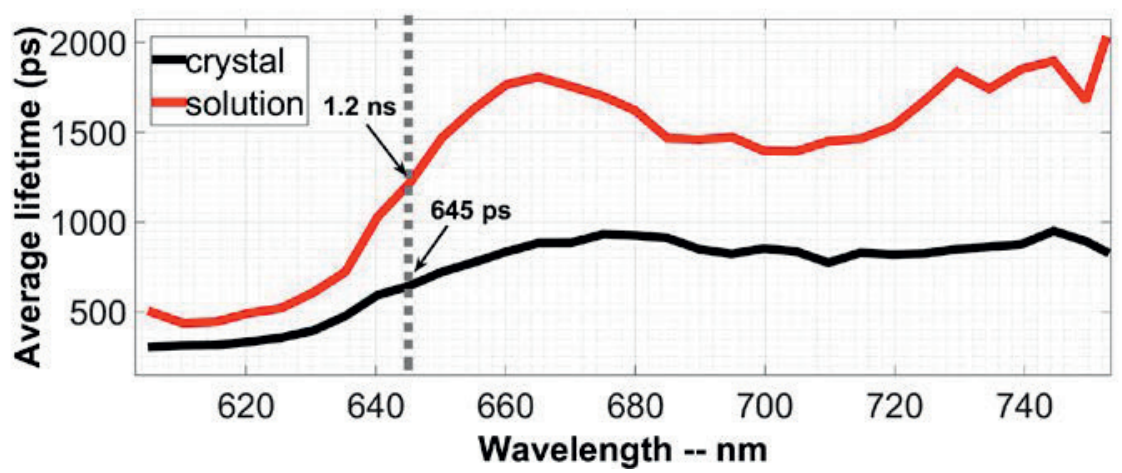

Fig. S3 The fluorescence lifetime of APC crystals and protein solution for different detection wavelengths. The lifetimes were calculated from the global analysis of streak-camera images using equation 2 in the main text. The vertical line shows the $645 \mathrm{~nm}$ wavelength that is also the detection wavelength in the FLIM image of Fig. 2 
Comparing the DAS of WT Synechocystis and those of APC in crystal and solution

Time-resolved fluorescence measurements in (Tian et al. 2011) on WT Synechocystis sp. PCC 6803 cells and in (Tian et al. 2012) on isolated PBS of WT Synechocystis sp. PCC 6803 and isolated PBS from a mutant lacking intermediary and core-distal C-PC hexamers (Ughy and Ajlani 2004) yielded DAS, similar to the 25 ps and 67 ps DAS in Fig. 3. The 39 ps DAS of whole cells (Tian et al. 2011), the 42 ps DAS of isolated WT PBS (Tian et al. 2012), and the 19 ps DAS of isolated PBS lacking the intermediary and core-distal C-PC hexamers (Tian et al. 2012) were assigned to EET from C-PC to APC 660 trimers. The similarity between the DAS of the APC protein solution and the 39 ps DAS of whole cells suggests that the latter can equally well be assigned to EET within APC 660 trimers, at least partly (see below). We also performed time-resolved measurements on WT Synechocystis sp. PCC 6803 and obtained a 43 ps DAS from the global analysis that again looks very similar to the 25 ps and 67 ps DAS of crystals and protein solution, respectively (see Fig. S4a). This further supports our suggestion that the 39 ps DAS in (Tian et al. 2011) is at least partly due to EET within APC trimers in the intact cells at room temperature. The difference in the EET lifetimes might be due to a difference in the interaction strength between $\mathrm{APCB}$ and $\beta \mathrm{PCB}$ in the different samples due to the different environments that all these proteins experience, i.e. they are either present in a crystal, solution or a phycobilisome, which might influence the degree of compaction or the number of neighbouring pigments to which EET can take place. 
To compare the shapes of the DAS obtained for APC crystals and APC in solution (see Fig. 3), we grouped different DAS and presented them in Fig. S4. In Fig. S4a the 25 ps and 67 ps DAS are normalized to the maximum of their corresponding time zero spectrum. The 43 ps DAS is multiplied by 0.11 to make its amplitude comparable to those of the other two. The rest of the DAS in Figs. S4b-c are normalized to their maximum. In Fig. S4d the time-zero spectrum is shown.

a)

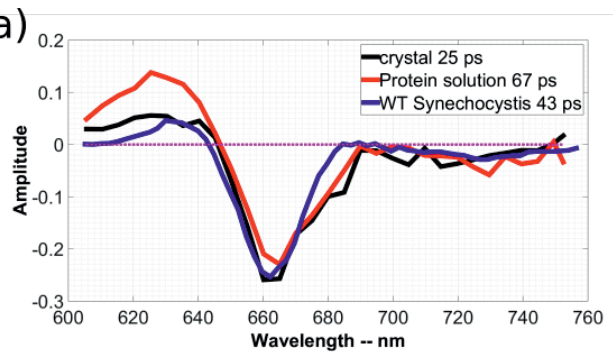

C)

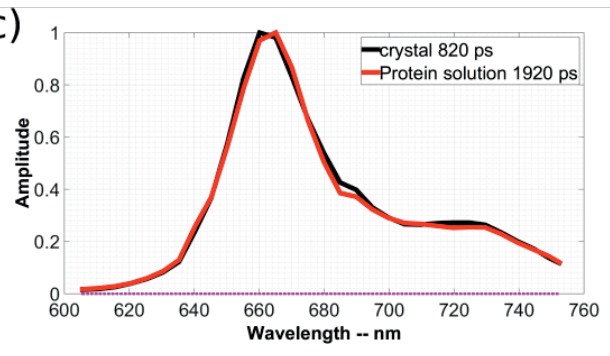

b)

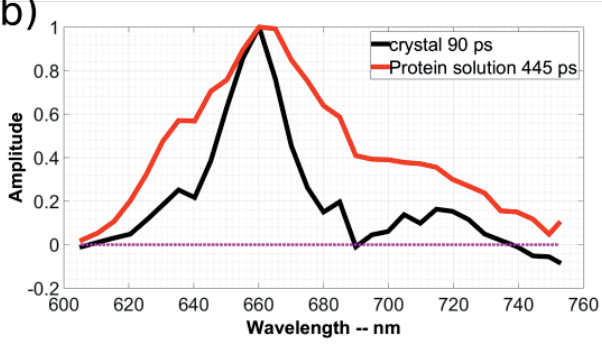

d)

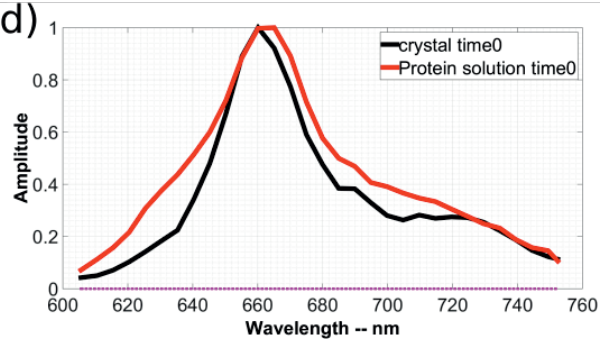

Fig. S4 a-c) Comparison between the DAS of APC crystals and protein solution. In a one of the DAS of WT Synechocystis sp. PCC 6803 as obtained with the streak camera is also presented. d) Time-zero spectrum of APC crystals and APC in solution

\section{References}

Tian L, Gwizdala M, van Stokkum IHM, et al (2012) Picosecond Kinetics of Light Harvesting and Photoprotective Quenching in Wild-Type and Mutant Phycobilisomes Isolated from the Cyanobacterium Synechocystis PCC 6803. Biophys J 102:1692-1700. doi: http://dx.doi.org/10.1016/j.bpj.2012.03.008

Tian L, Van Stokkum IHM, Koehorst RBM, et al (2011) Site, rate, and mechanism of photoprotective quenching in cyanobacteria. J Am Chem Soc 133:1830418311. doi: 10.1021/ja206414m

Ughy B, Ajlani G (2004) Phycobilisome rod mutants in Synechocystis sp. strain PCC6803. Microbiology 150:4147-4156. 
State transitions in the cyanobacterium Synechococcus elongatus 7942 involve reversible quenching of the photosystem II core

Reza Ranjbar Choubeh, Emilie Wientjes, Paul C.Struik, Diana Kirilovsky, Herbert van Amerongen, BBA - Bioenergetics (2018),

https://doi.org/10.1016/j.bbabio.2018.06.008 


\section{Abstract}

Cyanobacteria use chlorophyll and phycobiliproteins to harvest light. The resulting excitation energy is delivered to reaction centers (RCs), where photochemistry starts. The relative amounts of excitation energy arriving at the RCs of photosystem I (PSI) and II (PSII) depend on the spectral composition of the light. To balance the excitations in both photosystems, cyanobacteria perform state transitions to equilibrate the excitation energy. They go to state I if PSI is preferentially excited, for example after illumination with blue light (light I), and to state II after illumination with green-orange light (light II) or after dark adaptation. In this study, we performed 77$\mathrm{K}$ time-resolved fluorescence spectroscopy on wild-type Synechococcus elongatus 7942 cells to measure how state transitions affect excitation energy transfer to PSI and PSII in different light conditions and to test the various models that have been proposed in literature. The time-resolved spectra show that the PSIl core is quenched in state II and that this is not due to a change in excitation energy transfer from PSII to PSI (spill-over), either direct or indirect via phycobilisomes.

\section{Introduction}

The first step of photosynthesis is light harvesting (Croce and van Amerongen 2014) and in cyanobacteria, the light is harvested by chlorophyll a ( $\mathrm{Chl} \mathrm{a)} \mathrm{molecules} \mathrm{in}$ photosystems I and II (PSI and PSII) and bilins in the phycobilisomes (PBSs). The excited-state energy is delivered to the reaction centers (RCs) of both photosystems, where charge separation takes place and the subsequent photochemistry reactions lead to the production of biomass. PSI and PSII are embedded in the thylakoid membrane and both photosystems work in series. In addition, PBSs are used as accessory light-harvesting antennae that are mainly associated with PSII on the cytoplasmic side (Watanabe and Ikeuchi 2013). Depending on the spectral composition of the light, the relative amounts of energy arriving at the RCs of PSI and PSII can vary. The excitation energy can be redistributed between PSI and PSII, due to a change in the interaction between PBS, PSI, and PSII under redox control of inter-photosystem electron carriers, a process called state transitions (Kirilovsky 2015). State I is induced when PSI is preferentially excited (illumination by blue or far-red light (Kirilovsky 2015)) and hence the inter-photosystem electron carriers are 58 
State transitions in the cyanobacterium Synechococcus elongatus 7942 involve reversible quenching of the photosystem II core

more oxidized. State II is induced by preferential excitation of PSII (illumination by orange-green light) or by dark-adaptation (Kirilovsky 2015), which results in more reduction of the inter-photosystem electron carriers. State I (state II) is characterized by a high (low) fluorescence yield of PSIl at room temperature and a high (low) PSII/PSI emission ratio at $77 \mathrm{~K}$ (Kirilovsky 2015). While dark-adaptation induces state II and blue-light adaptation induces state I, other colors of light such as yelloworange bring the cells to a state between state I and II (Mullineaux and Allen 1990; Kirilovsky 2015).

Several mechanisms have been suggested for state transitions. One possible mechanism is the transfer of excitation energy from PSII to PSI, which is called spillover. According to the spill-over model (Bruce et al. 1989; Vernotte et al. 1990), excitation energy transfer (EET) takes place between Chl a molecules of PSI and PSII without any involvement of PBSs. Reversible migration of PBSs between PSI and PSII has also been used to explain state transitions and in this case only PBSs play a role (Mullineaux 1992). A combination of spill-over and slight PBS movement has also been suggested to regulate the energy (re)distribution (McConnell et al. 2002). In this model PBSs feed excitation energy to both PSI and PSII. The isolation of a functional cyanobacterial megacomplex that includes PSI, PSII and PBS may support this idea (Liu et al. 2013). In another study it was concluded that upon dark to light transitions a fraction of the PBSs decouples from PSI, but does not attach to PSII afterwards (Chukhutsina et al. 2015b).

In this study, we used time-resolved fluorescence measurements at $77 \mathrm{~K}$ to study state transitions in wild-type (WT) Synechococcus elongatus 7942 cells. Timeresolved fluorescence measurements have been used before to study light harvesting and its regulation in cyanobacteria (Bruce et al. 1985, 1986; Krumova et al. 2010; Tian et al. 2011, 2013; Chukhutsina et al. 2015a, b; van Stokkum et al. 2018). This study attempts (1) to reveal the differences in EET in state I and II and to verify/falsify existing models for state transitions; and (2) to assess whether state I-light II transitions and state II-light II transitions involve the same processes (light I/II is the light with spectral composition that preferentially excites Photosystem I/II). 


\section{Materials and Methods}

\section{Strains and Growth Conditions}

Wild-type Synechococcus elongatus 7942 and Synechocystis PCC 6803 cells were grown at $30^{\circ} \mathrm{C}$ in a medium containing $20 \mathrm{ml} / \mathrm{liter} \mathrm{BG}-11,0.85 \mathrm{~g} / \mathrm{liter}$ sodium bicarbonate, and $1.75 \mathrm{~g} /$ liter sodium nitrate at $\mathrm{pH}$ 8.0. A white light source with an intensity of $50 \mu \mathrm{mol}$ photons $\mathrm{m}^{-2} \mathrm{~s}^{-1}$ was used to illuminate the cells. The spectrum of the growth light is presented in Figure S1 in the SI. The cells were grown in 250 $\mathrm{ml}$ flasks with culture volume of $60 \mathrm{ml}$ that were shaken at $100 \mathrm{rpm}$.

\section{Sample Preparation}

The cells were grown in a fresh medium, starting at an optical density of 0.3 at 800 $\mathrm{nm}$ (OD800 scattering and $1 \mathrm{~cm}$ light path). For time-resolved spectroscopy measurements the cells were harvested at an $\mathrm{OD}_{800}$ of $\sim 0.55-0.70$. They were diluted with fresh medium to an $\mathrm{OD}_{630}$ of $\sim 0.30$, as measured with a spectrophotometer with integrating sphere.

The cells were pre-conditioned in four different conditions: dark (state II), dark-blue (State I), dark-orange (State II-to-Light II), and dark-blue-orange (State I-to-Light II). Dark-blue-orange means that after the cells were dark adapted for at least $\sim 15-20$ minutes, they were then illuminated by blue light for $\sim 20$ minutes, and subsequently with only orange light for $\sim 20$ minutes. Each stage of adaptation took $\sim 20$ minutes and the maximum fluorescence upon illumination of a saturating pulse (closing the $\mathrm{RCs}$ ) was monitored with the pulse-amplitude modulation (PAM) technique to make sure that cells reached an equilibrated state. A $440 \mathrm{~nm}$ (full with at half maximum (FWHM) of $40 \mathrm{~nm}$ ) filter and a $605 \mathrm{~nm}$ (FWHM of 30 or $50 \mathrm{~nm}$, both give the same result) filter were used for blue and orange light illumination. The intensity of either color of light was 50-65 $\mu \mathrm{mol}$ photons $\mathrm{m}^{-2} \mathrm{~s}^{-1}$ for Synechococcus elongatus 7942 and 35-50 $\mu \mathrm{mol}$ photons $\mathrm{m}^{-2} \mathrm{~s}^{-1}$ for Synechocystis PCC 6803. Samples were collected in a glass Pasteur pipette with a diameter of $\sim 1 \mathrm{~mm}$ and were frozen in liquid nitrogen for the fluorescence measurements.

\section{Steady-state absorption spectroscopy}


State transitions in the cyanobacterium Synechococcus elongatus 7942 involve reversible quenching of the photosystem II core

A Cary $5 E$ spectrophotometer, equipped with an integrating diffuse reflectance sphere (DRA-CA-50, Labsphere) was used to measure absorption spectra and correct for light scattering by the cells. To start growing the cells at $\mathrm{OD}_{800}$ of 0.3 and to harvest them at an $\mathrm{OD}_{800}$ of $\sim 0.55-0.70$, we did not use the integrating diffuse reflectance sphere.

\section{Steady-state fluorescence spectroscopy}

Steady-state fluorescence at $77 \mathrm{~K}$ was recorded with a Jobin Yvon Fluorolog FL322 spectrofluorimeter. The recorded spectrum was corrected for wavelengthdependent sensitivity of the detector and fluctuations in the lamp output. The excitation wavelengths were $577 \mathrm{~nm}$ and $430 \mathrm{~nm}$ with a bandwidth of $4.5 \mathrm{~nm}$. The detection bandwidth was $2 \mathrm{~nm}$. The spectra were averaged over several measurements.

\section{Time-resolved fluorescence spectroscopy}

Time-resolved fluorescence spectroscopy was performed using a picosecond streak-camera system as described before (van Stokkum et al. 2008; Tian et al. 2011; Chukhutsina et al. 2015b). 800 nm pulsed light from a Ti:sapphire laser (Coherent, Mira) was fed into a FemtoWHITE 800 tube (NKT photonics, part of Thorlabs' Supercontinuum generation kit). From its broad spectral output (470-700 $\mathrm{nm}$ ), light centered at $577 \mathrm{~nm}$ (FWHM of $10 \mathrm{~nm}$ ) was selected with a bandpass filter. For $430 \mathrm{~nm}$ excitation the frequency-doubled output of the Ti:sapphire laser (860 $\mathrm{nm}$ ) was used. The repetition rate of the laser was $3.8 \mathrm{MHz}$ with a power of $\sim 30 \mu \mathrm{W}$ for $577 \mathrm{~nm}$ and $\sim 100 \mu \mathrm{W}$ for $430 \mathrm{~nm}$ excitation. A lens with focal length of $\sim 7 \mathrm{~cm}$ was used to focus the light resulting in a spot size of $\sim 0.1 \mathrm{~mm}$ in both cases.

\section{Global analysis of time-resolved fluorescence data}

The fluorescence decay was recorded with 2000-ps and 800-ps time windows. The 800-ps time window has a better time resolution and allows to resolve the faster fluorescence decay processes. The 2000-ps time window allows for a better estimation of longer lifetimes and it was used to verify if the ns fluorescence decay component recorded with 800-ps time window was estimated correctly, which was 
indeed the case. Each image was corrected for the wavelength dependency of the detector and then sliced into $\sim 4 \mathrm{~nm}$ time traces.

Several measurements on dark-adapted cells (state II) were analyzed together so that all the measurements have the same lifetimes and decay-associated spectra (DAS); the DAS for different measurements on dark-adapted cells could only differ by a constant accounting for different levels of the fluorescence intensity. The same procedure was used for other measurements with the cells in the same condition, such as all measurements on blue-adapted cells (state I). The measurements performed on state I-to-Light II and state II-to-Light II were fitted with the same lifetimes while their amplitudes were fitted independent of each other. Global analysis, based on the singular-value decomposition (SVD) method, was performed as described in (Chukhutsina et al. 2015b), using the Glotaran (Snellenburg et al. 2012) and TIMP package (Mullen and van Stokkum 2007) for R. The instrument response function (IRF) of the streak-camera setup was described as a Gaussian function with its FWHM as a free fitting parameter. Global analysis of the data led to a FWHM of $\sim 11$ ps and $\sim 24$ ps at time windows 800 ps and 2000 ps, respectively. In all cases a fit with five components was required according to the SVD method and no meaningful fits with six components were possible, despite the high signalto-noise ratios. The quality of the fits is shown in the Supplementary material (Figures S2-S4).

\section{Results}

\section{Steady-state fluorescence spectroscopy}

In Figure 1, the steady-state fluorescence spectra of blue-light- and dark-adapted cells (state I and II, respectively) are shown. The spectra were recorded upon excitation with $577 \mathrm{~nm}$ (mainly PBS excitation) and $430 \mathrm{~nm}$ (mainly Chl a excitation) light, hereafter called $577 \mathrm{~nm}$ and $430 \mathrm{~nm}$ spectra. The $577 \mathrm{~nm}$ and $430 \mathrm{~nm}$ spectra were normalized to the peaks at $\sim 655 \mathrm{~nm}$ and $\sim 717 \mathrm{~nm}$, respectively. In order to normalize the spectra in an independent way, we also added fluorescein for $430 \mathrm{~nm}$ excitation ( $77 \mathrm{~K}$ fluorescence peak at $500 \mathrm{~nm}$ ) and rhodamine $B$ for $550 \mathrm{~nm}$ excitation (77 K fluorescence peak at $\sim 570 \mathrm{~nm}$ ) to compare the samples under different light 
State transitions in the cyanobacterium Synechococcus elongatus 7942 involve reversible quenching of the photosystem II core

conditions. Normalizing the recorded spectra to the fluorescence peaks of the added dyes yielded the same results as those shown in Figure 1 (see SI, Figures S5-S6). Upon $430 \mathrm{~nm}$ and $577 \mathrm{~nm}$ excitation four peaks were resolved that belong to Cphycocyanin (C-PC) and allophycocyanin (APC) (655 nm) (Kana et al. 2009), CP43 in PSII and the terminal emitters of PBS (APC680) (683 nm) (Mörschel and Schatz 1987; Andrizhiyevskaya et al. 2005; Kana et al. 2009), CP47 and the RCs of PSII (695 nm) (Mörschel and Schatz 1987; Andrizhiyevskaya et al. 2005; Kana et al. 2009), and to PSI (717 nm) (Andrizhiyevskaya et al. 2002).

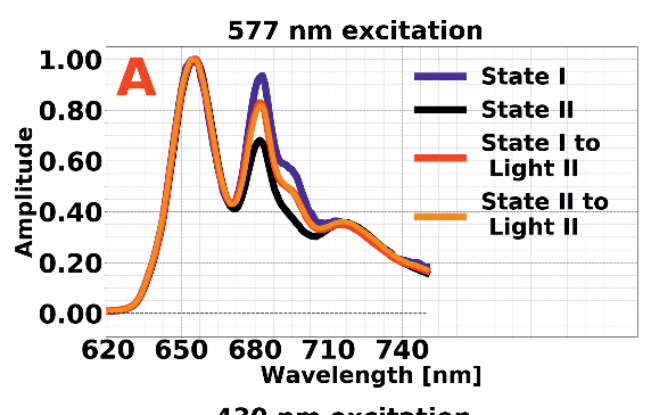

Steady-state spectra obtained both with $577 \mathrm{~nm}$ and $430 \mathrm{~nm}$ excitation light demonstrated that cells in state I showed a major increase of fluorescence at $683 \mathrm{~nm}$ (PBS and/or PSII) and $695 \mathrm{~nm}$ (PSII) when compared to cells in state II, while

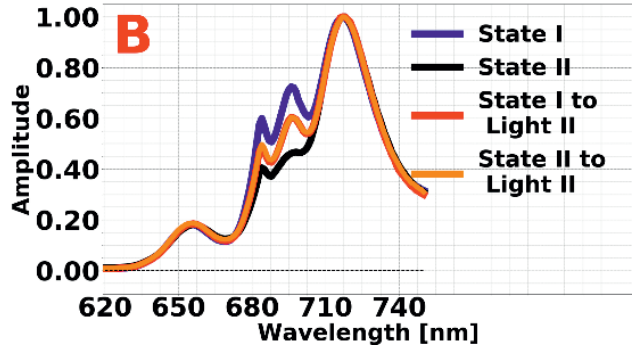

Figure 1. $77 \mathrm{~K}$ steady-state fluorescence spectra of WT Synechococcus elongatus 7942 cells adapted to different light conditions. The excitation wavelengths are indicated above each plot. The $577 \mathrm{~nm}$ (A) and 430 $\mathrm{nm}$ (B) spectra are normalized to the peak at $\sim 655 \mathrm{~nm}$ and $\sim 717 \mathrm{~nm}$, respectively (See Figures S5-6 for normalization to a fluorescent probe). In the legend "State I" and "State II" spectra correspond to cells that were blue-light- and dark-adapted. "State I to Light II" spectrum corresponds to cells that were initially dark-adapted, then illuminated by blue light (state I) and finally only by orange light (Light II). "State II to Light II" spectrum corresponds to cells that were initially dark-adapted and then were illuminated by orange light (light II). Note that the "State I to Light II" and "State II to Light II" spectra perfectly overlap. the fluorescence arising from phycobiliproteins and PSI remained unchanged (see SI Figures S5-S6). The lack of a change in PSI emission intensity suggests that its antenna size did not change during state transitions. However, we will demonstrate below that timeresolved fluorescence spectroscopy showed that the PSI antenna size changed to some extent due to detachment of PBSs in state I (see Discussion).

To elucidate if the processes involved in fluorescence changes 
are the same in state I-to-light II and state II-to-light II transitions, blue-light adapted cells (state I) and dark-adapted cells (state II) were both illuminated by orange light (light II). Figure 1 shows that, under our experimental conditions, the final state in both cases was identical and somewhere between state I and II; in other words, the final state was independent of the initial state. Probably, it depends only on the redox state of the $P Q$ pool under orange light, which in turn depends on the ratio of PSI to PSII and the relative energy transfer from PBS to PSI. Also picosecond fluorescence measurements on Synechococcus elongatus 7942 cells showed that, under our experimental conditions, the final state induced by illumination with orange light was independent of the starting state (Figure 4).

The next issues we addressed concerning state transitions were the origin of the fluorescence difference at $\sim 680-695 \mathrm{~nm}$ and the roles of PBSs and spill-over. For this we resort to time-resolved spectroscopy in the next section.

\section{Time-resolved fluorescence spectroscopy}

The fluorescence decay kinetics of cells in different states induced by blue, orange or dark adaptation were measured with the streak camera using $430 \mathrm{~nm}$ and/or 577 $\mathrm{nm}$ excitation at $77 \mathrm{~K}$. Global analysis of the data resulted in decay-associated spectra (DAS). Figure 2 shows the comparison of blue-light- (state I) and darkadapted (state II) cells excited by $430-\mathrm{nm}$ light (Chl a excitation).

All the DAS presented in this study are scaled according to the corresponding timezero spectrum. The time-zero spectrum is the fluorescence emission spectrum directly after excitation if no other relaxation processes occur and it is calculated by summing all the DAS obtained from global analysis.

Both the lifetime and spectral shape of the 4-ps DAS, Figure 2A, were very similar for both states. The negative peak of C-PC around 640-645 nm was due to EET from the high-energy pigments in C-PC to the lower-energy pigments (Tian et al. 2011). The positive peak at $\sim 685 \mathrm{~nm}$ was mainly due to short-wavelength $\mathrm{Chl} a$ in $\mathrm{PSI}$ and the negative signal between $\sim 700 \mathrm{~nm}$ and $\sim 800 \mathrm{~nm}$ corresponded to an increase of long-wavelength PSI emission. 
State transitions in the cyanobacterium Synechococcus elongatus 7942 involve reversible quenching of the photosystem II core

The second DAS, Figure $2 \mathrm{~B}$, had a lifetime of $\sim 12 \mathrm{ps}$ and the shape was very similar for states I and II. The positive peak at $\sim 640 \mathrm{~nm}$ and the negative peak at $\sim 670 \mathrm{~nm}$ reflected EET from C-PC to APC (Suter et al. 1984; Krumova et al. 2010; Tian et al. 2011). The peak at $\sim 690 \mathrm{~nm}$ belonged to PSII and those at $\sim 710 \mathrm{~nm}$ and $\sim 750 \mathrm{~nm}$ belonged to PSI.

Also the third DAS, Figure $2 \mathrm{C}$, with a lifetime of $\sim 52 \mathrm{ps}$, was very similar for both states. The fluorescence of C-PC was visible at $\sim 645 \mathrm{~nm}$. The peaks at $\sim 683 \mathrm{~nm}$ and $\sim 702 \mathrm{~nm}$ corresponded to $\mathrm{Chl}$ a of CP43 of PSIl and Chl a of PSI, respectively. The broad band around $~ 735-780 \mathrm{~nm}$ was also due to PSI. The first three DAS were rather similar for state I and II and they did not show any difference in PSI emission caused by spill-over from PSII.

The fourth DAS, Figure 2D, with a lifetime of $\sim 194 \mathrm{ps}$, contained peaks at $\sim 650 \mathrm{~nm}$ (C-PC), $\sim 683 \mathrm{~nm}$ (CP43), $\sim 710 \mathrm{~nm}$ (PSI) and $\sim 770 \mathrm{~nm}$ (PSI). The peak at $\sim 650 \mathrm{~nm}$ only showed a very small difference for state I and II and the PSI emission was also the same for both states. However, the CP43 emission ( $\sim 683 \mathrm{~nm})$ had a smaller amplitude in state II. The decrease of the amplitude in state II came at the expense of a rise of the amplitude of the shorter-lived DAS at the same wavelength. This rise could be observed when the first three DAS in state I were subtracted from the first three DAS in state II (Figure S7). The fact that the PSI emission was the same for states I and II argues against a change in spill-over from PSII to PSI.

The $5^{\text {th }}$ DAS with a lifetime of $1 \mathrm{~ns}$, Figure $2 \mathrm{E}$, had the same shape and amplitude for C-PC ( 650 nm) and PSI ( 720 nm) in state I and II, but the amplitude around 675-695 nm was higher for the state-I spectrum, which had some contribution from CP43, CP47, and (disconnected) PBSs.

All the DAS showed that the PSI emission did not differ for state I and II. This rules out the spill-over model as a way to re-distribute excitation energy during state transitions. The only difference between the DAS in states I and II arose from the PSII emission. PSII had a lower emission in state II while the PSI emission remained the same, which means that PSII was quenched in state II but not by PSI. 


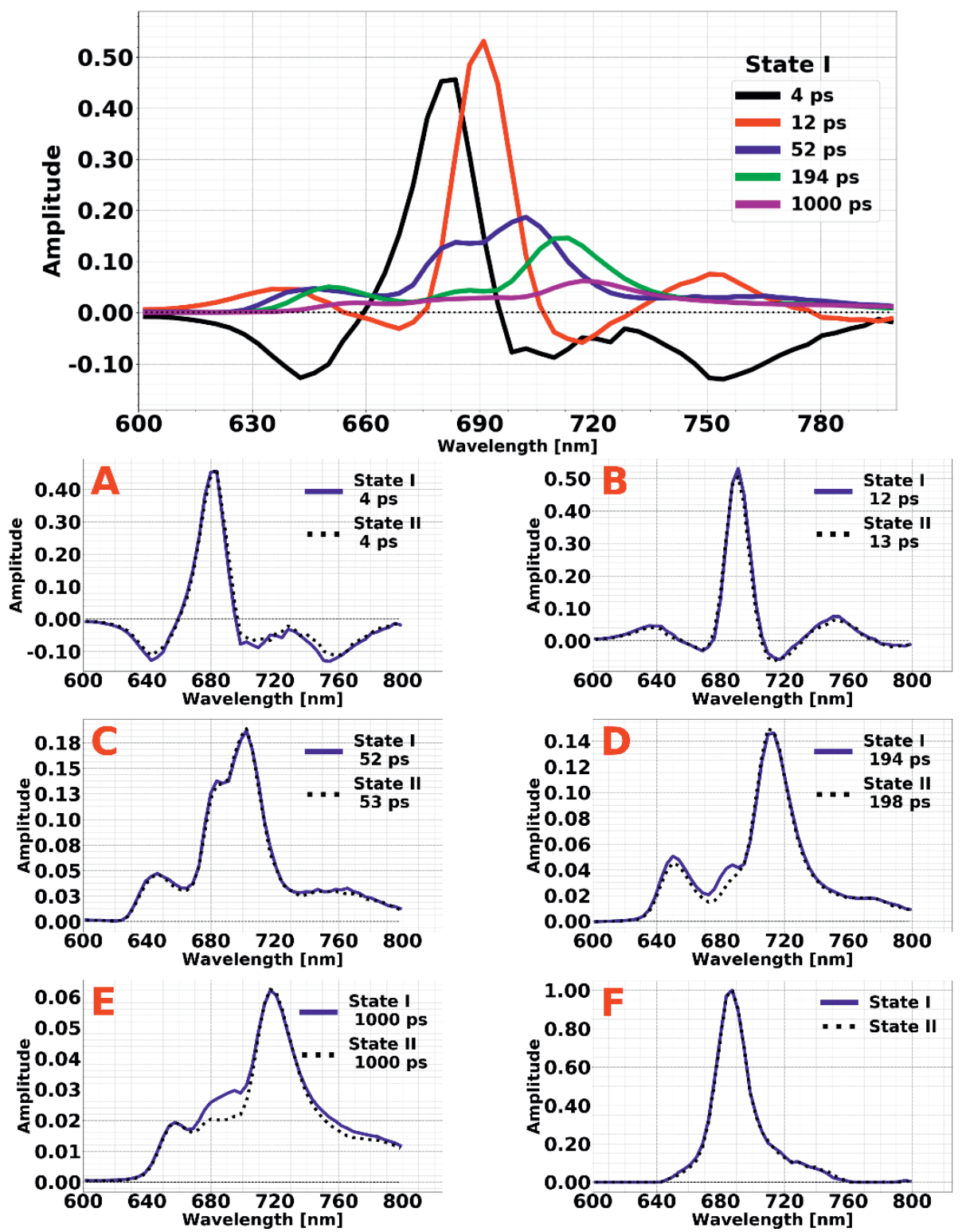

Figure 2. DAS obtained from global analysis of time-resolved measurements on WT Synechococcus elongatus 7942 cells in state I (blue-light adaptation) and state II (dark adaptation). The excitation wavelength is $430 \mathrm{~nm}$ (mainly $\mathrm{Chl}$ a excitation) and each DAS shown in A-E is scaled in accordance with the time-zero spectrum in $F$. To show the relative amplitude of different DAS, all the DAS in state I are shown on top. 
State transitions in the cyanobacterium Synechococcus elongatus 7942 involve reversible quenching of the photosystem II core

To investigate the role of PBSs in state transitions we used $577 \mathrm{~nm}$ excitation light for the time-resolved fluorescence measurements to mainly excite PBSs, and the results of the global analysis of the obtained data are given in Figure 3.

The first DAS, Figure 3A, was very similar for both states and had a lifetime of $\sim 9$ ps. Its positive and negative peaks at $\sim 625 \mathrm{~nm}$ and $\sim 650 \mathrm{~nm}$ indicate EET within the C-PC rods (Tian et al. 2011, 2012). We assign the positive peak of the 9 ps DAS at $685 \mathrm{~nm}$ to $\mathrm{Chl}$ a, which transfers its excitations to the long-wavelength pigments in PSI. The latter pigments are responsible for the negative band above $700 \mathrm{~nm}$.

The second DAS, Figure 3B, which was also similar for both states, had a lifetime of 26 ps and 21 ps in states I and II. This DAS with its positive and negative peaks at $\sim 640 \mathrm{~nm}$ and $\sim 680 \mathrm{~nm}$ reflected EET from C-PC to APC680.

The third DAS, Figure 3C, had a lifetime of $85 \mathrm{ps}$ and $75 \mathrm{ps}$ in states I and II, respectively. There were three peaks at $\sim 645 \mathrm{~nm}, \sim 680 \mathrm{~nm}$ and $\sim 705 \mathrm{~nm}$. We assign the $\sim 645 \mathrm{~nm}$ peak to C-PC, the $\sim 680 \mathrm{~nm}$ peak to APC680 and $\mathrm{Chl}$ a, and the peak at $\sim 705 \mathrm{~nm}$ to PSI. This DAS reflects decay of excitation energy in C-PC, APC680, and $\mathrm{Chl}$ a. Both DAS had the same amplitude at $\sim 645 \mathrm{~nm}$, but the DAS in state II had a higher amplitude at $\sim 680 \mathrm{~nm}$. This rise came at the expense of the decrease in amplitude of the long-lifetime DAS at the same wavelength (figures 3D-E) and was due to quenching of PSII (see Discussion).

The fourth DAS, Figure 3D, had lifetimes of $285 \mathrm{ps}$ and $242 \mathrm{ps}$ in states I and II, respectively. They had peaks at $\sim 650 \mathrm{~nm}$ (C-PC), $\sim 683 \mathrm{~nm}$ (PSII), and $\sim 710 \mathrm{~nm}$ (PSI). The peak at $\sim 650 \mathrm{~nm}$ did not differ for state I and state II. The peak at $\sim 683$ $\mathrm{nm}$ (PSII) had a lower amplitude in state II, which was due to quenching of PSII in state II. The amplitude at $\sim 695 \mathrm{~nm}$ (PSII) and $\sim 710 \mathrm{~nm}$ (PSI) were somewhat lower in state I, which we ascribed to detachment of some PBSs from PSI and PSII in state I. 

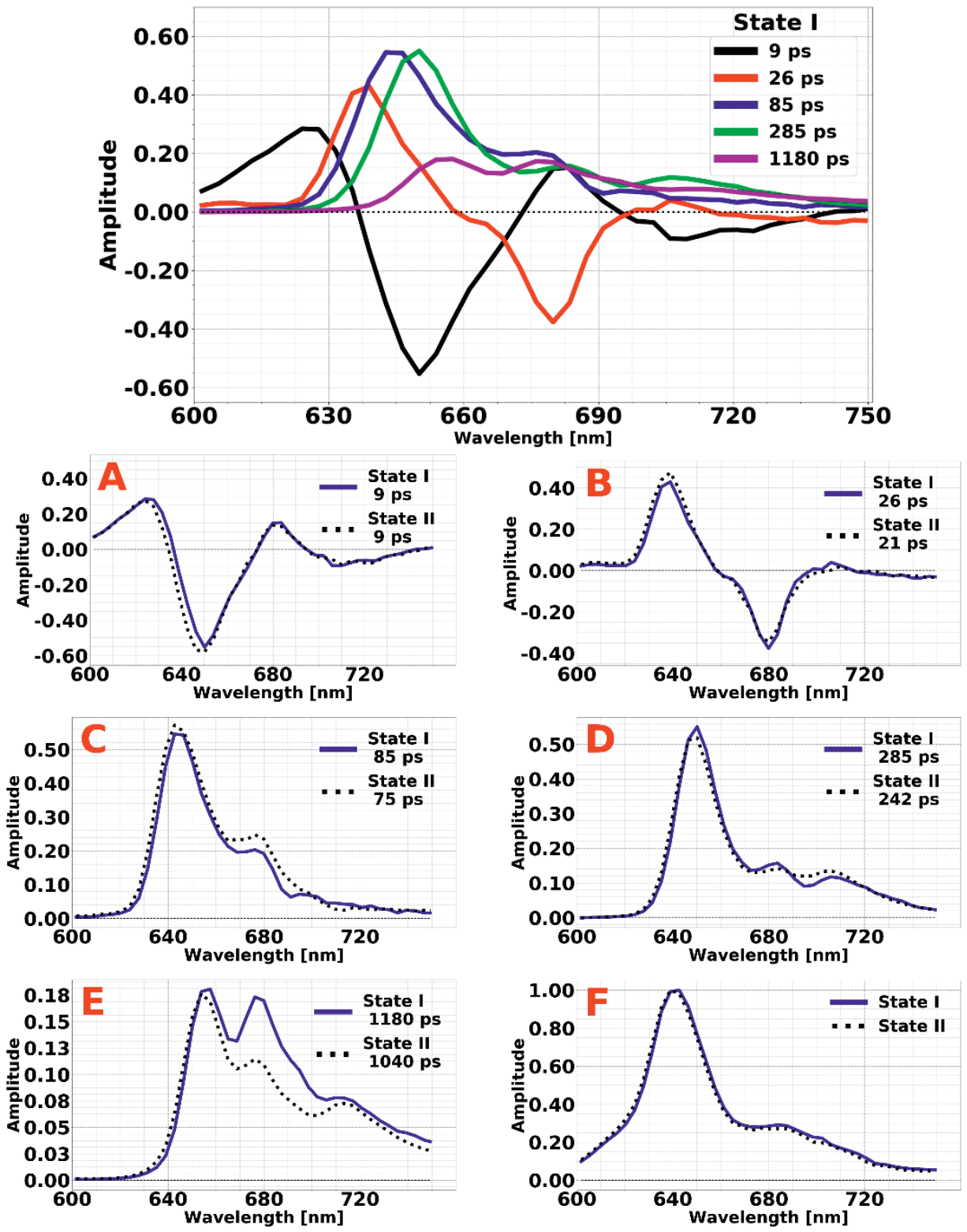

Figure 3. DAS obtained from global analysis of time-resolved measurements at $77 \mathrm{~K}$ on Synechococcus elongatus 7942 cells in state I (blue-light adaptation) and state II (dark adaptation). The excitation wavelength is $577 \mathrm{~nm}$ (mainly PBS excitation) and each DAS shown in A-E is scaled in accordance with the time-zero spectrum in $F$. To show the relative amplitude of different DAS, all the DAS in state I are shown on top. 
State transitions in the cyanobacterium Synechococcus elongatus 7942 involve reversible quenching of the photosystem II core

The fifth DAS, Figure 3E, had lifetimes of 1180 ps and 1040 ps in states I and II, respectively. The peaks at $\sim 655 \mathrm{~nm}$ (C-PC) and $\sim 715 \mathrm{~nm}$ (PSI) were almost the same for states I and II. However, in state I the peak at $\sim 683 \mathrm{~nm}$ (PBS last emitter and CP43) was much higher than in state II. PSII was quenched in state II and some PBSs detached from PSI and PSII in state I, which resulted in a rise of the fluorescence at $\sim 683-695 \mathrm{~nm}$ in state I as compared to state II (see Discussion).

In Figure 4, we compare two groups of WT Synechococcus elongatus 7942 cells that were adapted to orange light (light II) but had a different light treatment history for cells in which the final light treatment was the same. In one group, the cells were initially dark-adapted (state II) and then illuminated with orange light. In the second group, the cells were initially dark-adapted, then illuminated with blue light (state I), and finally with only orange light. The final "orange" state appeared to be independent of the initial state. This result was confirmed by a comparison of the corresponding steady-state spectra, which also showed that the final state was independent of the initial state (see Figure 1). 

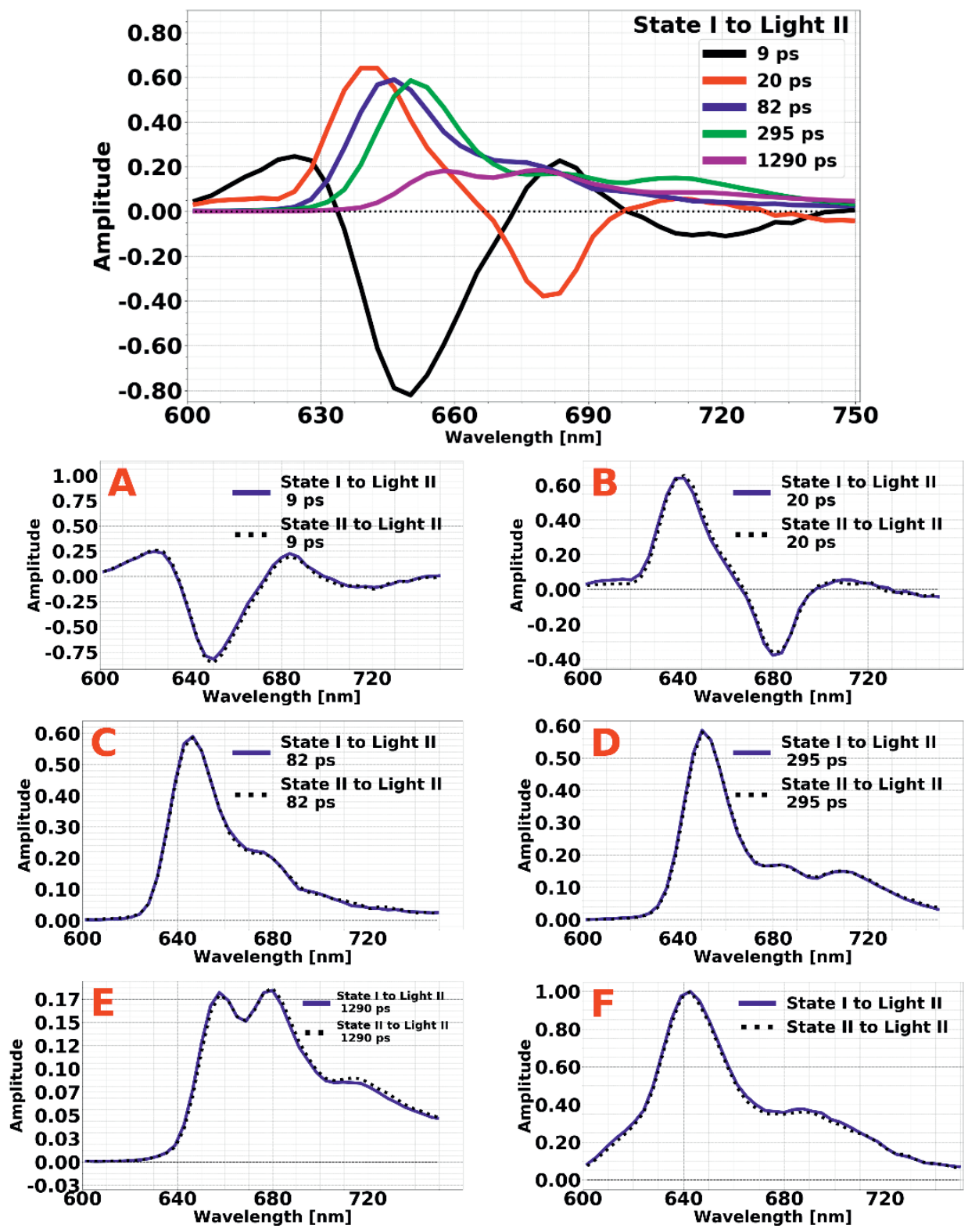

Figure 4. DAS obtained from global analysis of time-resolved measurements on orange-adapted (Light II) Synechococcus elongatus 7942 cells. In one case, the cells were initially dark-adapted (State II to Light II) and in the other they were blue-light adapted before illumination by light II (State I to Light II). The excitation wavelength is $577 \mathrm{~nm}$ (mainly PBS excitation) and each DAS shown in $A-E$ is scaled in accordance with the time-zero spectrum in $F$. The lifetimes of the two measurements were set to be the same during analysis. To show the relative amplitude of different DAS, all the DAS in state I-to-Light II are shown on top. 
State transitions in the cyanobacterium Synechococcus elongatus 7942 involve reversible quenching of the photosystem II core

\section{Discussion}

There are several models that attempt to explain state transitions in cyanobacteria. We present the two main models and discuss how our data rule out or confirm different aspects of each of these models.

\section{Spill-over model}

In the spill-over model, excitation energy spills over from PSII to PSI in state II, either after direct excitation of PSII or after excitation of PBSs that transfer their excitation energy to PSII, from where it moves to PSI. Spill-over from PSII to PSI must thus lead to an increase in PSI fluorescence in state II as compared to state I and the same is true for the ratio of PBS/PSI and PSII/PSI fluorescence. While the spill-over model can explain the changes in the ratio of PSII/PSI emission observed both upon excitation of PBSs and excitation of $\mathrm{Chl}$ a, it does not explain why the PSI emission and PBS/PSI emission ratio do not differ for state I and II (see Figures 1 and 2). This indicates that either there is no EET from PSII to PSI or, if any spill-over occurs, it is not involved in state transitions and happens regardless of the state in which the cells are present. Also, the time-resolved measurements on Synechocystis PCC 6803 cells show that the PSI emission was the same for state I and II (see Figure S8).

In the literature a variety of observations has been reported regarding PSI fluorescence in state I and state II. In (Olive et al. 1997; El Bissati et al. 2000; McConnell et al. 2002) $77 \mathrm{~K}$ steady-state spectra of Synechocystis PCC 6803 and Synechococcus 7002 (normalized either to PSI emission, PBS emission, or an internal fluorescent probe) showed that the ratio of PBS/PSI emission remained the same in state I and II, which is in agreement with our results (see Figure 1). However, in (Bruce et al. 1989; Salehian and Bruce 1992) the $77 \mathrm{~K}$ spectra of Synechococcus PCC 7002 and Synechococcus PCC 6301 cells showed an increase of PSI emission in state II upon PBS or Chl a excitation. This is in disagreement with the results of (Olive et al. 1997; El Bissati et al. 2000; McConnell et al. 2002) and our results that showed the PBS/PSI emission ratio does not differ in state I and II. The Gaussian 
decomposition of the steady-state spectra in Figure 1B for cells in state I and II (ChI a excitation) and that of the DAS in Figure 2D-E (Chl a excitation) did not show any significant change in PSI emission in state I and II (see Figures S9-S11).

In (Bruce et al. 1985), $77 \mathrm{~K}$ time-resolved measurements were performed on the cyanobacterium Anacystis nidulans (excitation at $590 \mathrm{~nm}$ ). The authors found that the PSII decay time was faster in state II, whereas the decay times of PBS and PSI emission were not substantially affected. This was interpreted as being consistent with the spill-over model and to be in disagreement with a mobile PBS model. Also, our results on Synechococcus elongatus 7942 showed that upon PBS excitation the PSII lifetime was shorter in state II (Figure S12). However, our results demonstrated that the shorter lifetime of PSII was not due to quenching of PSII by PSI.

\section{Migration of PBSs between PSI and PSII}

It has been proposed before (Joshua 2004; Kaňa et al. 2014) that PBSs migrate between PSI and PSII during state transitions. This migration cannot explain the large changes in the ratio of the PSII/PSI emission observed upon $430 \mathrm{~nm}$ excitation when PBSs were excited to a far lesser extent than $\mathrm{Chl}$ a (Figures 1B and 2). In the latter case we need to ascribe the large difference to PSII quenching in state II. In Figure 3D (PBS excitation), the emission of PSI decreased in state I by $18 \%$ (See the Gaussian decomposition in Figure S13). The emission of PSII at $694 \mathrm{~nm}$ was also lower in state I (Figure S13). The Gaussian decomposition yielded a non-zero contribution to the Gaussian function at $694 \mathrm{~nm}$ in state II, while in state I its contribution became zero. This indicates that in state I some PBSs were detached from both PSI and PSII. The detachment of PBSs from PSI and PSII was accompanied by an increase of emission in the DAS in Figure 3E in state I (PBS excitation) at $\sim 680 \mathrm{~nm}$. In principle this might either be due to an increase of PBS or PSIl emission. To distinguish between these two possibilities, we performed a Gaussian decomposition of the DAS in Figures 2E (Chl a excitation) and 3E (PBS excitation), which resolved two Gaussians in the 670-695 $\mathrm{nm}$ region for each DAS (Figures S11 and S14). The DAS in Figures 2E and 3E had the Gaussians centered at $680 \mathrm{~nm}, 694 \mathrm{~nm}$ and at $678 \mathrm{~nm}, 694 \mathrm{~nm}$, respectively. In state I, the amplitude ratio of the two Gaussians (G678/G694) of the DAS in Figure 3E (PBS excitation 72 
State transitions in the cyanobacterium Synechococcus elongatus 7942 involve reversible quenching of the photosystem II core

excitation) was far larger than the amplitude ratio of the corresponding Gaussians (G680/G694) of the DAS in Figure 2E (Chl a excitation). This higher amplitude ratio must be ascribed to extra APC680 emission upon PBS excitation as compared to $\mathrm{Chl}$ a excitation because of the detachment of PBSs.

The fact that PSII becomes substantially quenched in state II as compared to state I might suggest that this would lead to extra heat formation in state II. However, previous optoacoustic studies on Synechococcus sp. PCC 6301, did not show significant differences on heat production for state I and state II (Mullineaux et al. 1991; Bruce and Salehian 1992). This cyanobacteria strain showed similar differences in $77 \mathrm{~K}$ fluorescence from cells in state I and state II as we observed here for Synechococcus elongatus 7942 also suggesting a quenched PS II (Bruce and Salehian 1992). On the other hand, in state I part of the PBSs now appears to be disconnected from the photosystems and instead of delivering their excitation energy to the RCs, they fall back to the ground state, and a substantial part of the excitation energy is transformed into heat. This might explain why heat production is similar for state I and II.

\section{Conclusions}

The most important result of this study is that state transitions are not due to a change in spill-over or to reshuffling of PBSs between PSI and PSII in Synechococcus elongatus 7942. Instead, PSII becomes substantially quenched in state II as compared to state I. PSII quenching can also be observed in Synechocystis PCC 6803, but on a much smaller scale. Apparently, this compensates for the over-reduction of the inter-photosystem electron carriers in the case of preferential excitation of PSII as compared to PSI. This quenching is different from the photoprotective non-photochemical quenching (NPQ) process that occurs in high-light conditions. Here the quenching occurs at the level of the PSIl core whereas NPQ takes place at the level of the PBSs under the influence of lightinduced switching of the orange carotenoid protein (OCP) (Kirilovsky 2015). 


\section{Acknowledgments}

This work was funded by The Netherlands Organization for Scientific Research (NWO) (project number 10TBSC24-3). This project was carried out within the research programme of BioSolar Cells, co-financed by the Dutch Ministry of Economic Affairs. We thank Rob Koehorst and Cor Wolfs for their support and their help in growing the cells and Dr Arjen Bader for his technical support during the timeresolved measurements.

\section{References}

Acuña AM, Lemaire C, van Grondelle R, et al (2018) Energy transfer and trapping in Synechococcus WH 7803. Photosynth Res 135:115-124. doi:

$10.1007 / \mathrm{s} 11120-017-0451-2$

Andrizhiyevskaya EG, Chojnicka A, Bautista JA, et al (2005) Origin of the F685 and F695 fluorescence in Photosystem II. Photosynth Res 84:173-180. doi: $10.1007 / \mathrm{s} 11120-005-0478-7$

Andrizhiyevskaya EG, Schwabe TME, Germano M, et al (2002) Spectroscopic properties of PSI-IsiA supercomplexes from the cyanobacterium Synechococcus $\{$ PCC 7942. Biochim Biophys Acta - Bioenerg 1556:265272. doi: http://doi.org/10.1016/S0005-2728(02)00371-7

Bruce D, Biggins J, Steiner T, Thewalt M (1985) Mechanism of the light state transition in photosynthesis. IV. Picosecond fluorescence spectroscopy of Anacystis nidulans and Porphyridium cruentum in state 1 and state 2 at $77 \mathrm{~K}$. Biochim Biophys Acta - Bioenerg 806:237-246. doi: https://doi.org/10.1016/0005-2728(85)90101-X

Bruce D, Brimble S, Bryant DA (1989) State transitions in a phycobilisome-less mutant of the cyanobacterium Synechococcus sp. PCC 7002. Biochim Biophys Acta - Bioenerg 974:66-73. doi: https://doi.org/10.1016/S00052728(89)80166-5 
State transitions in the cyanobacterium Synechococcus elongatus 7942 involve reversible quenching of the photosystem II core

Bruce D, Hanzlik CA, Hancock LE, et al (1986) Energy distribution in the photochemical apparatus of Porphyridiumcruentum: Picosecond fluorescence spectroscopy of cells in state 1 and state 2 at $77 \mathrm{~K}$. Photosynth Res 10:283290. doi: 10.1007/BF00118292

Bruce D, Salehian O (1992) Laser-induced optoacoustic calorimetry of cyanobacteria. The efficiency of primary photosynthetic processes in state 1 and state 2. Biochim Biophys Acta - Bioenerg 1100:242-250. doi: https://doi.org/10.1016/0167-4838(92)90478-V

Chukhutsina V, Bersanini L, Aro E-M, van Amerongen H (2015a) Cyanobacterial flv4-2 Operon-Encoded Proteins Optimize Light Harvesting and Charge Separation in Photosystem II. Mol Plant 8:747-761. doi: https://doi.org/10.1016/j.molp.2014.12.016

Chukhutsina V, Bersanini L, Aro E-M, van Amerongen H (2015b) Cyanobacterial Light-Harvesting Phycobilisomes Uncouple From Photosystem I During DarkTo-Light Transitions. Sci Rep 5:14193. doi: 10.1038/srep14193

Croce R, van Amerongen H (2014) Natural strategies for photosynthetic light harvesting. Nat Chem Biol 10:492-501

El Bissati K, Delphin E, Murata N, et al (2000) Photosystem II fluorescence quenching in the cyanobacterium Synechocystis PCC 6803: involvement of two different mechanisms. Biochim Biophys Acta - Bioenerg 1457:229-242. doi: http://dx.doi.org/10.1016/S0005-2728(00)00104-3

Joshua S (2004) Phycobilisome Diffusion Is Required for Light-State Transitions in Cyanobacteria. Plant Physiol 135:2112-2119. doi: 10.1104/pp.104.046110

Kaňa R, Kotabová E, Lukeš M, et al (2014) Phycobilisome Mobility and Its Role in the Regulation of Light Harvesting in Red Algae. Plant Physiol 165:1618 LP1631

Kana R, Prásil O, Komárek O, et al (2009) Spectral characteristic of fluorescence 
induction in a model cyanobacterium, Synechococcus sp. (PCC 7942).

Biochim Biophys Acta 1787:1170-1178. doi: 10.1016/j.bbabio.2009.04.013

Kirilovsky D (2015) Modulating energy arriving at photochemical reaction centers:

Orange carotenoid protein-related photoprotection and state transitions.

Photosynth Res 126:3-17. doi: 10.1007/s11120-014-0031-7

Krumova SB, Laptenok SP, Borst JW, et al (2010) Monitoring Photosynthesis in Individual Cells of Synechocystis sp. PCC 6803 on a Picosecond Timescale. Biophys J 99:2006-2015. doi: https://doi.org/10.1016/j.bpj.2010.07.015

Liu H, Zhang H, Niedzwiedzki DM, et al (2013) Phycobilisomes Supply Excitations to Both Photosystems in a Megacomplex in Cyanobacteria. Science (80- ) 342:1104 LP-1107

McConnell MD, Koop R, Vasil'ev S, Bruce D (2002) Regulation of the distribution of chlorophyll and phycobilin-absorbed excitation energy in cyanobacteria. A structure-based model for the light state transition. Plant Physiol 130:12011212. doi: $10.1104 / p p .009845$

Mörschel E, Schatz GH (1987) Correlation of photosystem-II complexes with exoplasmatic freeze-fracture particles of thylakoids of the cyanobacterium Synechococcus sp. Planta 172:145-154. doi: 10.1007/BF00394582

Mullen KM, van Stokkum IHM (2007) TIMP: An R Package for Modeling Multi-way Spectroscopic Measurements. J Stat Softw 18:200-6. doi:

10.1359/JBMR.0301229

Mullineaux CW (1992) Excitation energy transfer from phycobilisomes to Photosystem I in a cyanobacterium. Biochim Biophys Acta - Bioenerg 1100:285-292. doi: https://doi.org/10.1016/0167-4838(92)90483-T

Mullineaux CW, Allen JF (1990) State 1-State 2 transitions in the cyanobacterium Synechococcus 6301 are controlled by the redox state of electron carriers between Photosystems I and II. Photosynth Res 23:297-311. doi: 10.1007/BF00034860 
State transitions in the cyanobacterium Synechococcus elongatus 7942 involve reversible quenching of the photosystem II core

Mullineaux CW, Griebenow S, Braslavsky SE (1991) Photosynthetic energy storage in cyanobacterial cells adapted to light-states 1 and 2. A laserinduced optoacoustic study. Biochim Biophys Acta - Bioenerg 1060:315-318. doi: https://doi.org/10.1016/S0005-2728(05)80323-8

Olive J, Ajlani G, Astier C, et al (1997) Ultrastructure and light adaptation of phycobilisome mutants of Synechocystis PCC 6803. Biochim Biophys Acta Bioenerg 1319:275-282. doi: https://doi.org/10.1016/S0005-2728(96)00168-5

Salehian O, Bruce D (1992) Distribution of excitation energy in photosynthesis: quantification of fluorescence yields from intact cyanobacteria. J Lumin 51:91-98. doi: https://doi.org/10.1016/0022-2313(92)90021-Z

Snellenburg JJ, Laptenok SP, Seger R, et al (2012) Glotaran : A Java -Based Graphical User Interface for the R Package TIMP. J Stat Softw 49:. doi: 10.18637/jss.v049.i03

Suter GW, Mazzola P, Wendler J, Holzwarth AR (1984) Fluorescence decay kinetics in phycobilisomes isolated from the bluegreen alga Synechococcus 6301. Biochim Biophys Acta - Bioenerg 766:269-276. doi: https://doi.org/10.1016/0005-2728(84)90241-X

Tian L, Gwizdala M, van Stokkum IHM, et al (2012) Picosecond Kinetics of Light Harvesting and Photoprotective Quenching in Wild-Type and Mutant Phycobilisomes Isolated from the Cyanobacterium Synechocystis PCC 6803. Biophys J 102:1692-1700. doi: http://dx.doi.org/10.1016/j.bpj.2012.03.008

Tian L, Van Stokkum IHM, Koehorst RBM, et al (2011) Site, rate, and mechanism of photoprotective quenching in cyanobacteria. J Am Chem Soc 133:1830418311. doi: $10.1021 / \mathrm{ja} 206414 \mathrm{~m}$

Tian L, van Stokkum IHM, Koehorst RBM, van Amerongen H (2013) Light Harvesting and Blue-Green Light Induced Non-Photochemical Quenching in Two Different C-Phycocyanin Mutants of Synechocystis PCC 6803. J Phys 
Chem B 117:11000-11006. doi: 10.1021/jp309570u

van Stokkum IHM, Gwizdala M, Tian L, et al (2018) A functional compartmental model of the Synechocystis PCC 6803 phycobilisome. Photosynth Res 135:87-102. doi: 10.1007/s11120-017-0424-5

van Stokkum IHM, van Oort B, van Mourik F, et al (2008) (Sub)-Picosecond Spectral Evolution of Fluorescence Studied with a Synchroscan StreakCamera System and Target Analysis. In: Aartsma TJ, Matysik J (eds) Biophysical Techniques in Photosynthesis. Springer Netherlands, Dordrecht, pp 223-240

Vernotte C, Astier C, Olive J (1990) State 1-state 2 adaptation in the cyanobacteria Synechocystis PCC 6714 wild type and Synechocystis PCC 6803 wild type and phycocyanin-less mutant. Photosynth Res 26:203-212. doi:

10.1007/BF00033133

Watanabe M, Ikeuchi M (2013) Phycobilisome: Architecture of a light-harvesting supercomplex. Photosynth Res 116:265-276. doi: 10.1007/s11120-0139905-3 
State transitions in the cyanobacterium Synechococcus elongatus 7942 involve reversible quenching of the photosystem II core

\section{Supplementary Information}

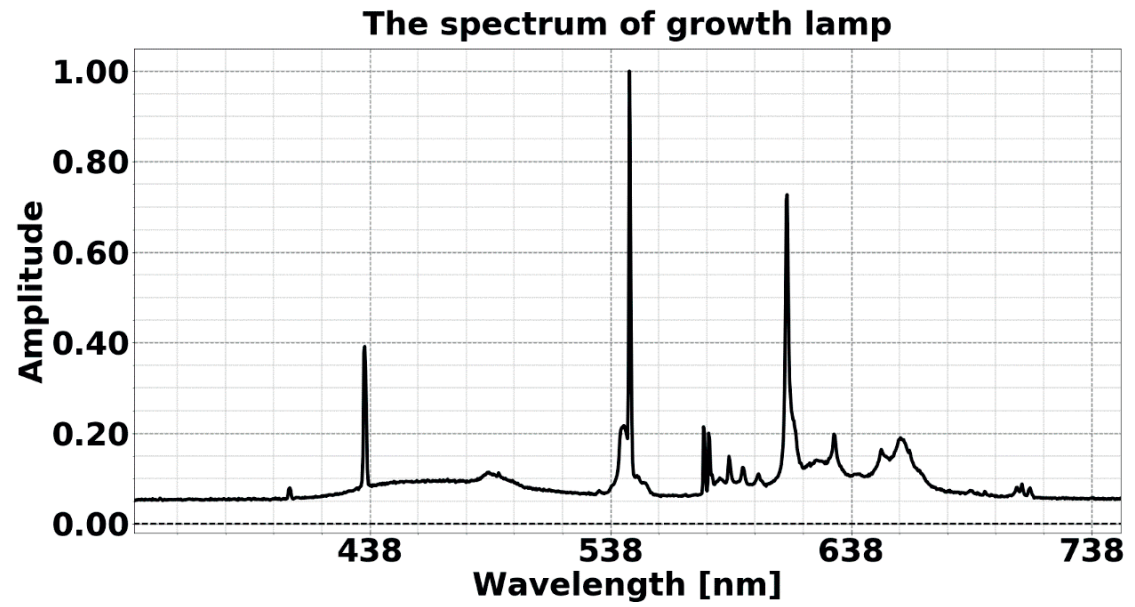

Figure S2. The spectrum of the lamp that was used to grow Synechococcus elongatus 7942 and Synechocystis PCC 6803 cells.
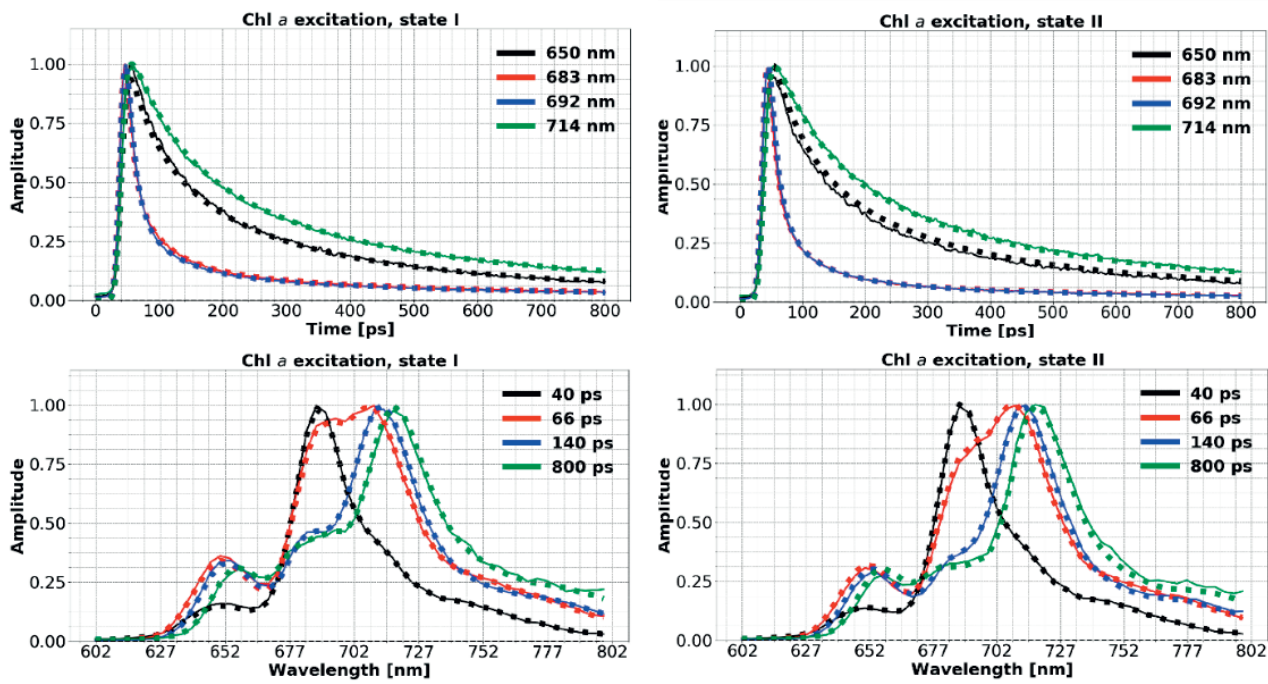

Figure S2. The measured time-traces at different wavelengths and spectra at different times are shown alongside their fit. The fit and measured data are taken from the data for which its global analysis is presented in Figure 2. The solid lines are the experimental data and the dotted lines are the fit. The time-traces at $683 \mathrm{~nm}$ and $692 \mathrm{~nm}$ overlap in state II. 

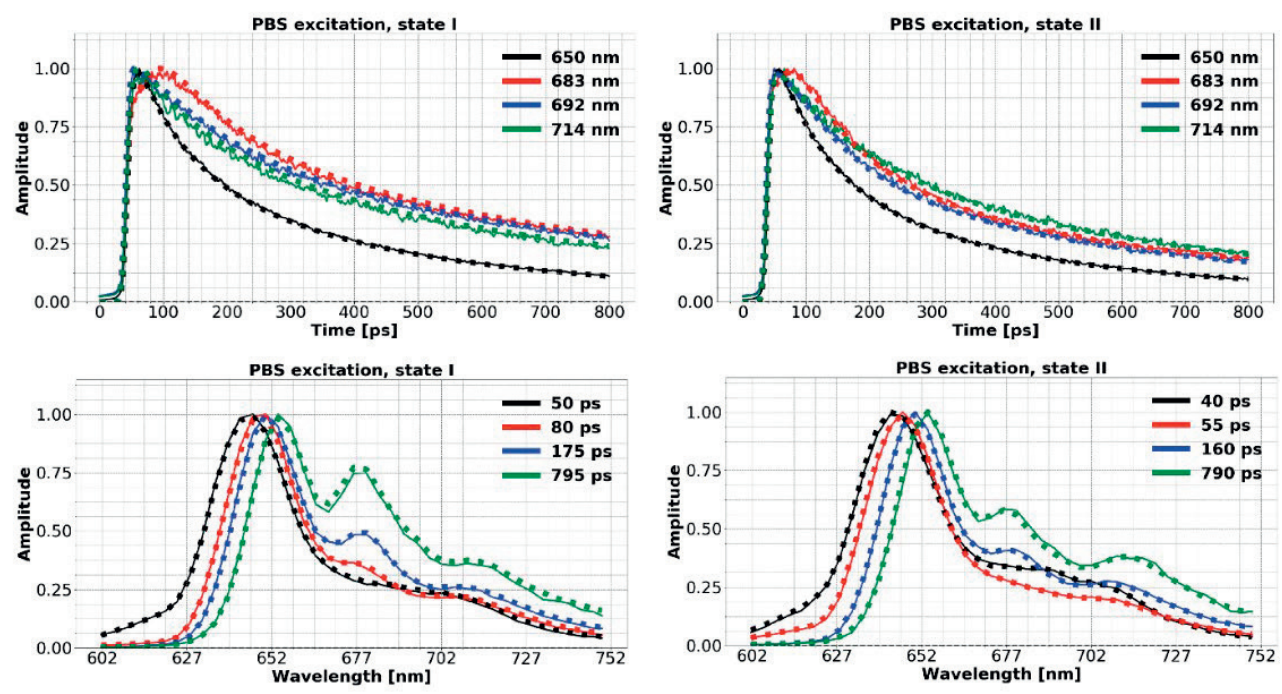

Figure S3. The measured time-traces at different wavelengths and spectra at different times are shown alongside their fit. The fit and measured data are taken from the data for which its global analysis is presented in Figure 3. The solid lines are the experimental data and the dotted lines are the fit.
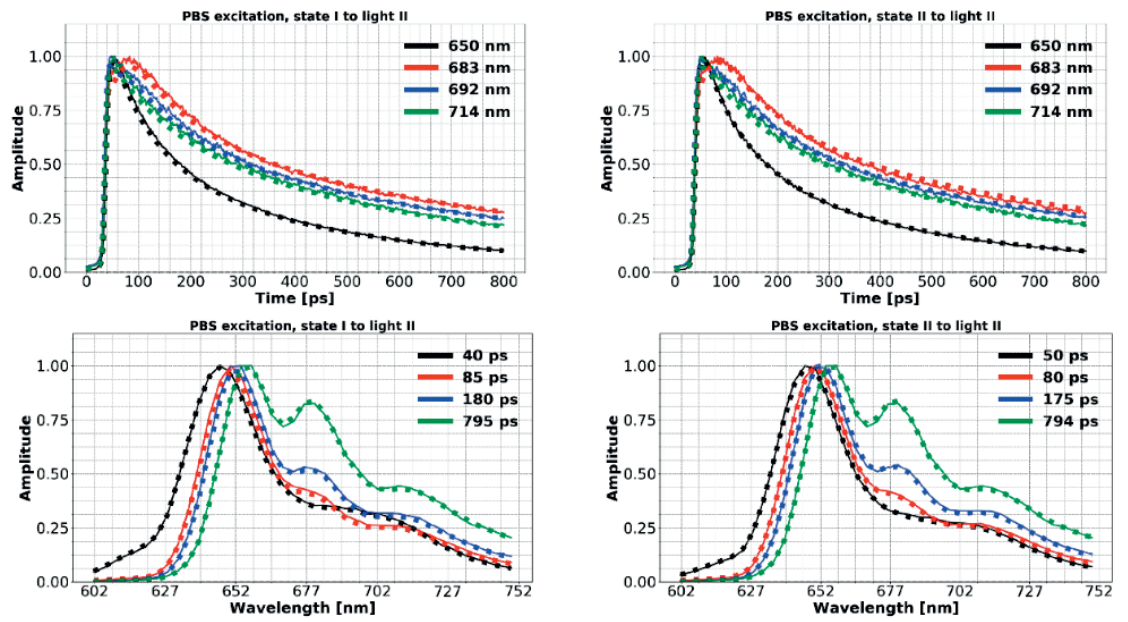

Figure S4. The measured time-traces at different wavelengths and spectra at different times are shown alongside their fit. The fit and measured data are taken from the data for which its global analysis is presented in Figure 4. The solid lines are the experimental data and the dotted lines are the fit. 


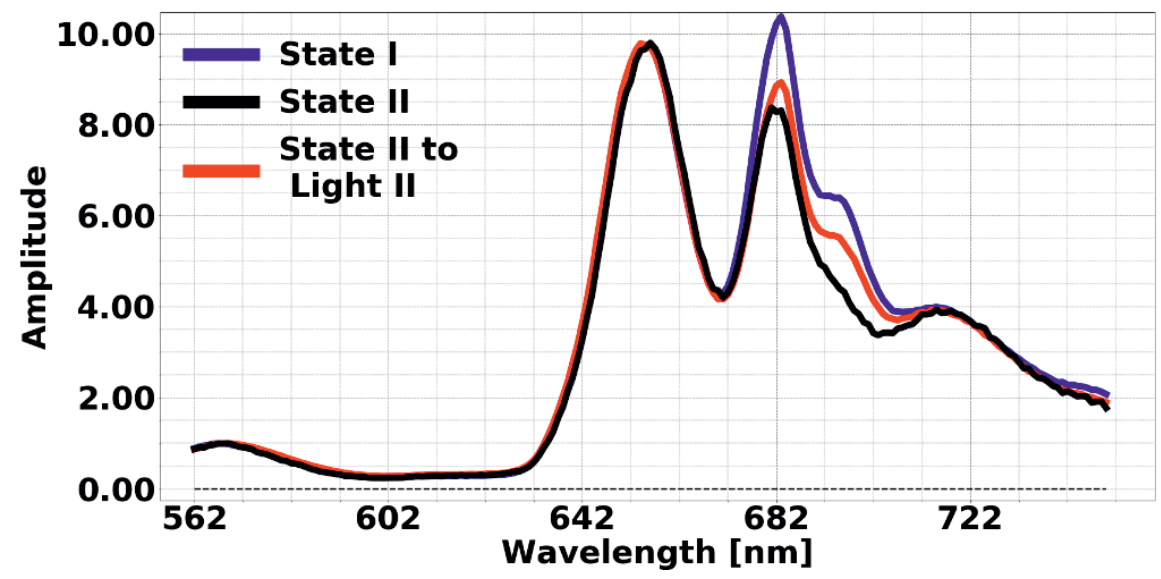

Figure S5. Usage of rhodamine B as an external probe for $77 \mathrm{~K}$ fluorescence steady-state spectra of WT Synechococcus elongatus 7942 cells adapted to different light conditions. The excitation wavelength is $550 \mathrm{~nm}$ and the spectra are normalized to the peak of rhodamine B at $\sim 570 \mathrm{~nm}$. In the legend, "State I" and "State II" spectra correspond to the cells that were adapted to blue light and darkness, respectively. "State II to Light II" corresponds to cells that were initially darkadapted (state II) and then illuminated by orange light (light II).

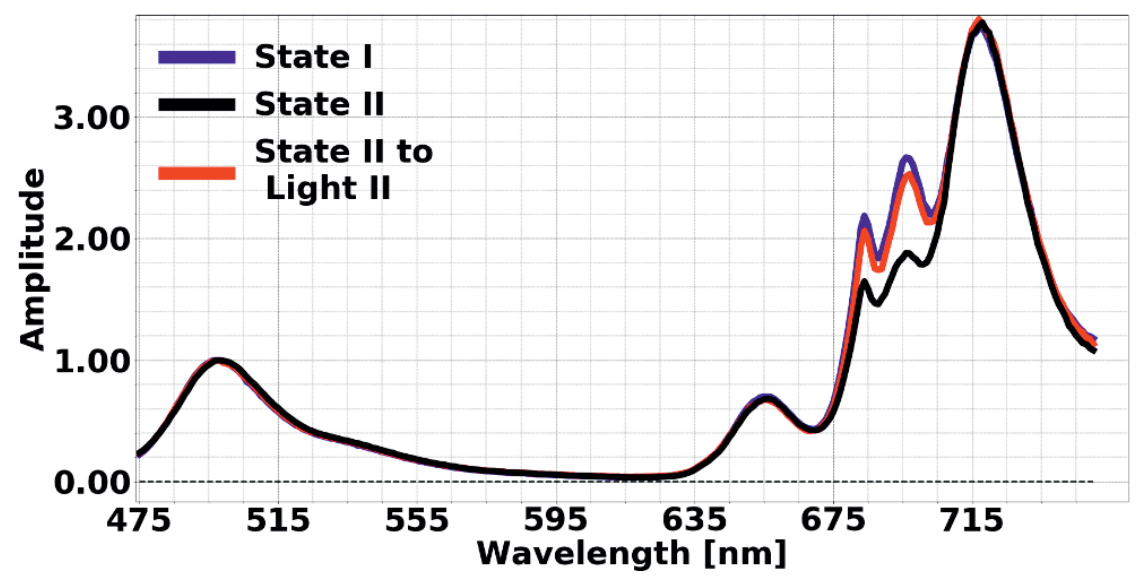

Figure S6. Usage of fluorescein as an external probe for $77 \mathrm{~K}$ fluorescence steady-state spectra of WT Synechococcus elongatus 7942 cells adapted to different light conditions. The excitation wavelengths is $430 \mathrm{~nm}$ and the spectra are normalized to the fluorescein peak at $\sim 500 \mathrm{~nm}$. In the legend, "State I" and "State II" spectra correspond to the cells that were adapted to blue light and darkness, respectively. "State II to Light II" corresponds to cells that were initially dark-adapted (state II) and then illuminated by orange light (light II). 


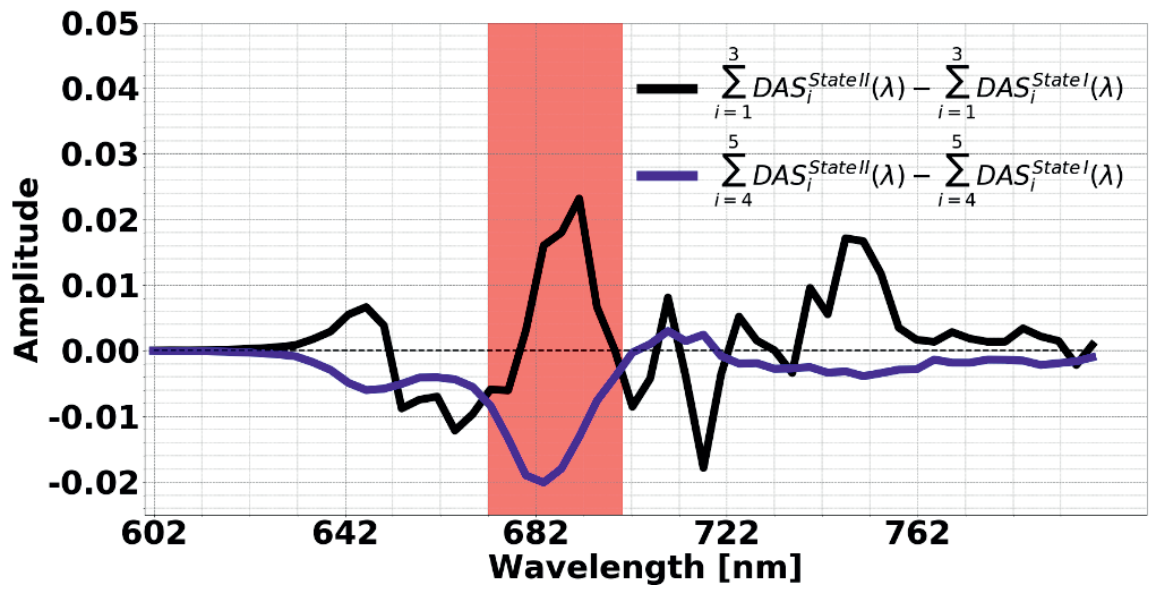

Figure S7. The first three DAS of Figure 2 ( $\mathrm{Chl}$ a excitation and Synechococcus elongatus 7942 cells ) in state I are subtracted from the first three DAS in state II. Also, the sum of the $4^{\text {th }}$ and $5^{\text {th }}$ DAS in state I is subtracted from the sum of the $4^{\text {th }}$ and $5^{\text {th }}$ DAS in state II. The decrease of the amplitude in state II in $4^{\text {th }}$ and $5^{\text {th }}$ DAS comes at the expense of a rise in the fast lifetime DAS (the first three DAS). 
State transitions in the cyanobacterium Synechococcus elongatus 7942 involve reversible quenching of the photosystem II core
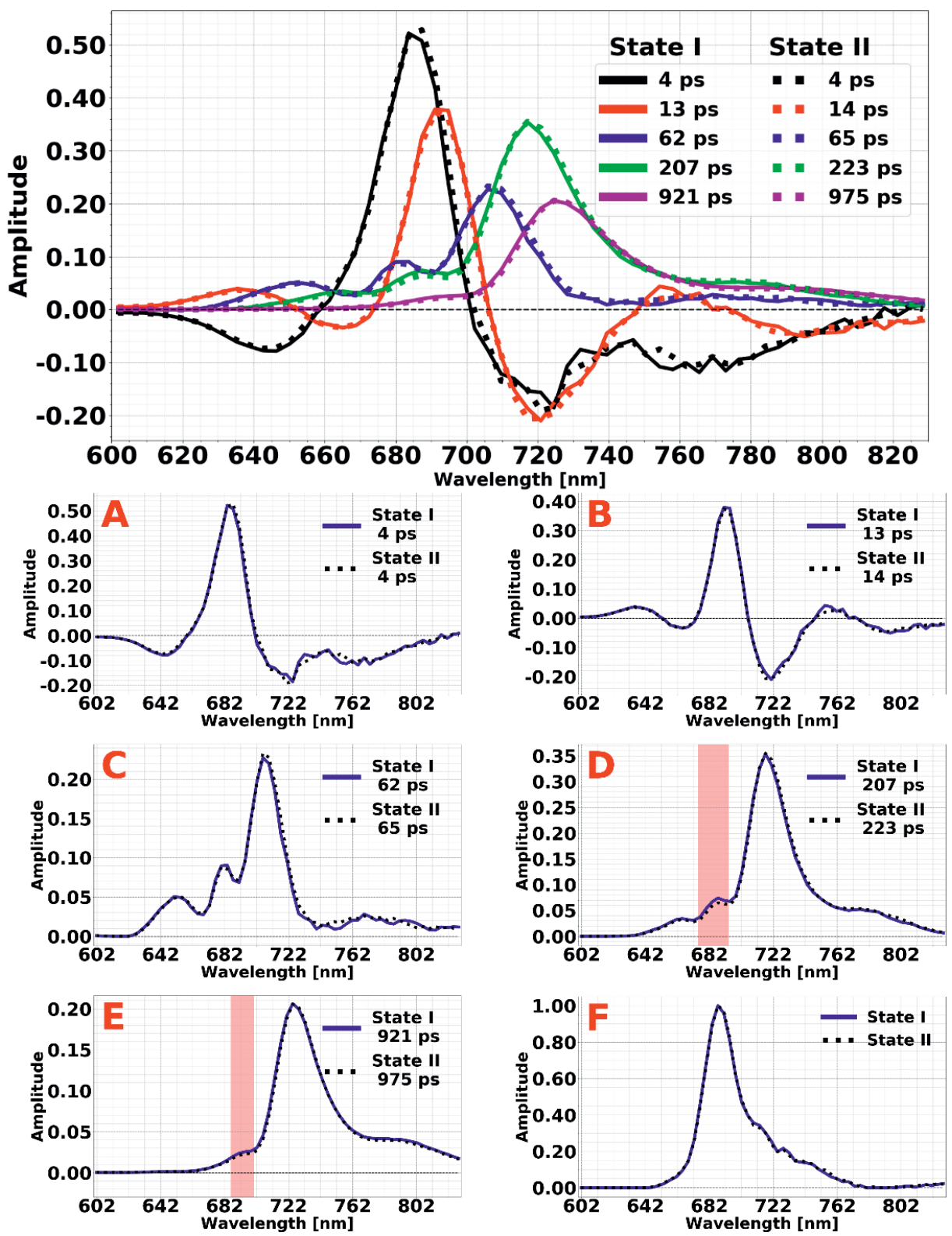

Figure S8. DAS obtained from global analysis of time-resolved measurements on blue-light (state I) and dark-adapted (state II) Synechocystis PCC 6803 cells. The excitation wavelength is $430 \mathrm{~nm}$ (mainly Chl a excitation) and each DAS shown in A-E is scaled in accordance with the time-zero spectrum in $\mathbf{F}$. 


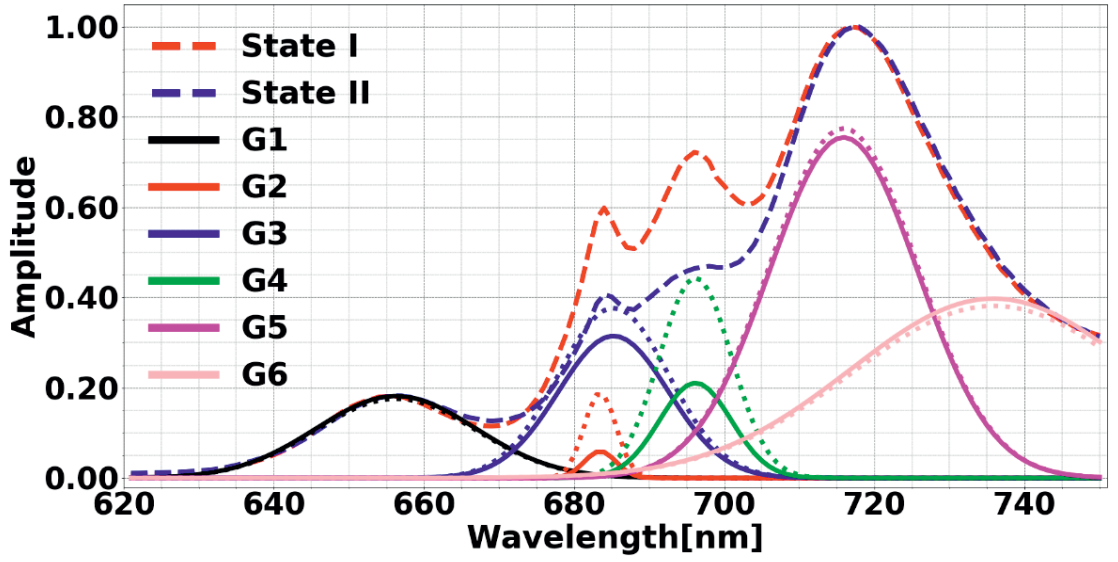

Figure S9. The Gaussian decomposition of the steady state spectrum of Synechococcus elongatus 7942 cells (Chl a excitation). The dotted (solid) Gaussians represent the Gaussians in state I (state II). The dashed red and blue curves show the steady-state spectra in state I and II from Figure 1B.

Figure S9 shows the Gaussian dcomposition of the $77 \mathrm{~K}$ steady-state spectrum of dark- and blue light adapted cells in Figure 1B. In order to describe the steady-state spectra, which contain the contributions of many different species 6 Gaussian functions are needed. The decomposition shows that the difference in PSI emission for state I and state II is negligible.

In Table 1 the details of each Gaussian function are shown. The differences were calculated with respect to state I:

$$
\begin{aligned}
& \text { Absolute difference }=\text { Area }_{\text {State II }}-\text { Area }_{\text {State I }} \text { eq. } 1 \\
& \text { Percentage change }=\frac{\text { Area }_{\text {State II }}-\text { Area }_{\text {State I }}}{\text { Area State I }} \quad \text { eq. } 2
\end{aligned}
$$

Table 1. The values describing the Gaussian functions in \$9 and their changes in state I and state II.

\begin{tabular}{|c|c|c|c|c|}
\hline & $\begin{array}{l}\text { Position } \\
\text { in } \mathrm{nm}\end{array}$ & $\begin{array}{l}\text { FWHM } \\
\text { in } \mathrm{nm}\end{array}$ & $\begin{array}{l}\text { Percentage change in } \\
\text { steady state } \\
\text { spectrum (Figure 1B) }\end{array}$ & $\begin{array}{l}\text { Absolute change in } \\
\text { steady state } \\
\text { spectrum (Figure } \\
1 B \text { ) }\end{array}$ \\
\hline $\begin{array}{c}\text { Gaussian } \\
1\end{array}$ & 656 & 25.0 & 3 & 0.16 \\
\hline $\begin{array}{c}\text { Gaussian } \\
2\end{array}$ & 683 & 5.0 & -69 & -0.69 \\
\hline
\end{tabular}


State transitions in the cyanobacterium Synechococcus elongatus 7942 involve reversible quenching of the photosystem II core

\begin{tabular}{|c|c|c|c|c|}
\hline $\begin{array}{c}\text { Gaussian } \\
3\end{array}$ & 685 & 17.3 & -16 & -1.14 \\
\hline $\begin{array}{c}\text { Gaussian } \\
4\end{array}$ & 696 & 11.3 & -53 & -2.80 \\
\hline $\begin{array}{c}\text { Gaussian } \\
5\end{array}$ & 716 & 23.3 & -3 & -0.50 \\
\hline $\begin{array}{c}\text { Gaussian } \\
6\end{array}$ & 736 & 44.8 & 4 & 0.78 \\
\hline
\end{tabular}

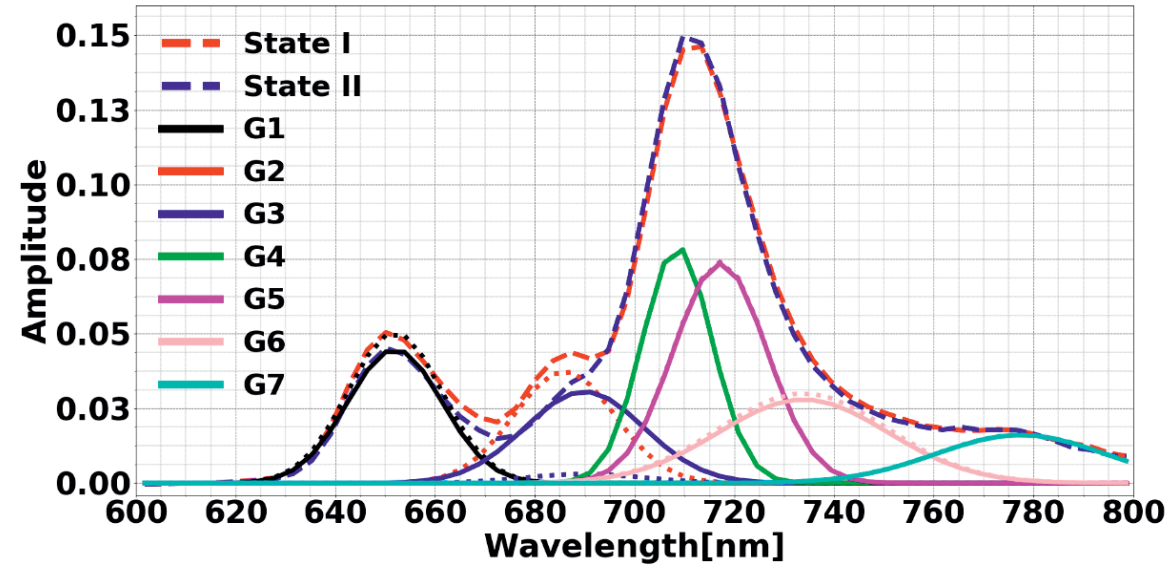

Figure S10. The Gaussian decomposition of the 4th DAS (Chl a excitation) of Synechococcus elongatus 7942 cells. The dotted (solid) Gaussians represent the Gaussians in state I (state II). The dashed red and blue curves show the DAS in state I and II from Figure 2D. Notice that the amplitude of G2 in state II is zero and G3 has a small amplitude in state I. The Gaussians G4 and G5 do not change in state I and II.

In Table 2 the details of each Gaussian function in Figure S10 are shown. The differences were calculated using Equations 1 and 2. In the main text it was mentioned that the total emission of PSII decreases by $19 \%$ in state II. To calculate this we used Equation 1, then Area $_{\text {State II }}$ was the total area of Gaussian functions representing PSII in state II. These Gaussian functions are G2 and G3 in Figure S10 and Figure S11. 
Table 2 The values describing the Gaussian functions in Figure S10 and their changes in state I and state II.

\begin{tabular}{|c|c|c|c|c|}
\hline & $\begin{array}{l}\text { Position } \\
\text { in nm }\end{array}$ & $\begin{array}{l}\text { FWHM } \\
\text { in nm }\end{array}$ & $\begin{array}{l}\text { Percentage change in } \\
\mathbf{4}^{\text {th }} \text { DAS (Figure 2D) }\end{array}$ & $\begin{array}{l}\text { Absolute change in } \\
\mathbf{4}^{\text {th }} \text { DAS (Figure } \\
\text { 2D) }\end{array}$ \\
\hline $\begin{array}{c}\text { Gaussian } \\
1\end{array}$ & 652 & 22.3 & -11 & -0.13 \\
\hline $\begin{array}{c}\text { Gaussian } \\
2\end{array}$ & 686 & 23.8 & $*$ & 0 \\
\hline $\begin{array}{c}\text { Gaussian } \\
3\end{array}$ & 690 & 28.1 & * & -0.01 \\
\hline $\begin{array}{c}\text { Gaussian } \\
4\end{array}$ & 709 & 16.5 & -1 & -0.09 \\
\hline $\begin{array}{c}\text { Gaussian } \\
5\end{array}$ & 717 & 22.0 & -7 & 0 \\
\hline $\begin{array}{c}\text { Gaussian } \\
6\end{array}$ & 734 & $40.0^{* *}$ & 0 & \\
\hline $\begin{array}{c}\text { Gaussian } \\
7\end{array}$ & 777 & $40.0^{* *}$ & 0.95 and & \\
\hline
\end{tabular}

* In state I the amplitudes of G2 and G3 were 0.95 and 0.09. In state II the amplitude of $\mathrm{G} 2$ became zero and that of G3 became 0.92 .

** The maximum allowed value for the FWHM of the Gaussian function during fitting was $40 \mathrm{~nm}$.

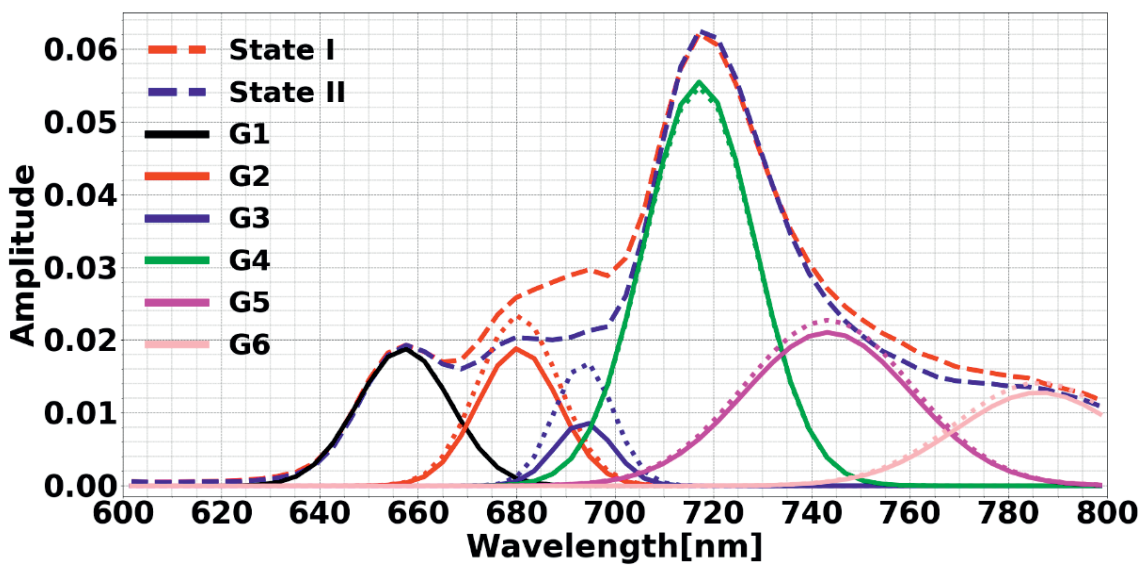

Figure S11. The Gaussian decomposition of the 5th DAS (Chl a excitation) of Synechococcus elongatus 7942 cells. The dotted (solid) Gaussians represent the Gaussians in state I (state II). The dashed red and blue curves show the DAS in state I and II from figure 2E.

In Table 3 the details of each Gaussian function in Figure S11 are shown. The differences were calculated using Equations 1 and 2 . 
State transitions in the cyanobacterium Synechococcus elongatus 7942 involve reversible quenching of the photosystem II core

Table 3 The values describing the Gaussian functions in S11 and their changes in state I and state II.

\begin{tabular}{|c|c|c|c|c|}
\hline & $\begin{array}{l}\text { Position } \\
\text { in nm }\end{array}$ & $\begin{array}{l}\text { FWHM } \\
\text { in nm }\end{array}$ & $\begin{array}{l}\text { Percentage change in } \\
\mathbf{5}^{\text {th }} \text { DAS (Figure 2E) }\end{array}$ & $\begin{array}{l}\text { Absolute change in } \\
\mathbf{5}^{\text {th }} \text { DAS (Figure } \\
\text { 2E) }\end{array}$ \\
\hline $\begin{array}{c}\text { Gaussian } \\
1\end{array}$ & 657 & 22.3 & 0 & 0 \\
\hline $\begin{array}{c}\text { Gaussian } \\
2\end{array}$ & 680 & 19.3 & $-20^{*}$ & -0.09 \\
\hline $\begin{array}{c}\text { Gaussian } \\
3\end{array}$ & 694 & 14.0 & $-49^{*}$ & 0.02 \\
\hline $\begin{array}{c}\text { Gaussian } \\
4\end{array}$ & 717 & 26.4 & 1 & -0.07 \\
\hline $\begin{array}{c}\text { Gaussian } \\
5\end{array}$ & 743 & $40.0^{* *}$ & -7 & -0.06 \\
\hline $\begin{array}{c}\text { Gaussian } \\
6\end{array}$ & 786 & $40.0^{* *}$ & -10 & \\
\hline
\end{tabular}

* The amplitudes of G2 in state I and II are 0.48 and 0.39 , respectively. The amplitudes of $\mathrm{G} 3$ in state I and II are 0.25 and 0.13 , respectively.

** The maximum FWHM allowed in the fitting was $40 \mathrm{~nm}$. 


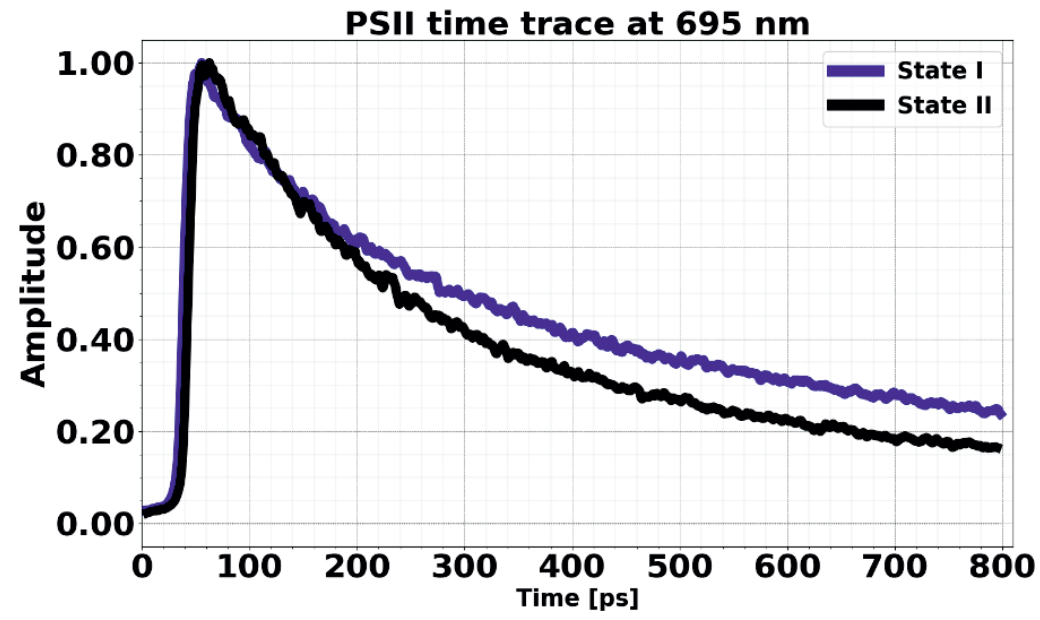

Figure S12. Time trace of PSII fluorescence at $695 \mathrm{~nm}(6 \mathrm{~nm}$ FWHM) in state I and state II. The time traces correspond to Synechococcus elongatus 7942 cells with PBS excitation (577 nm). In state II PSII has a shorter lifetime.

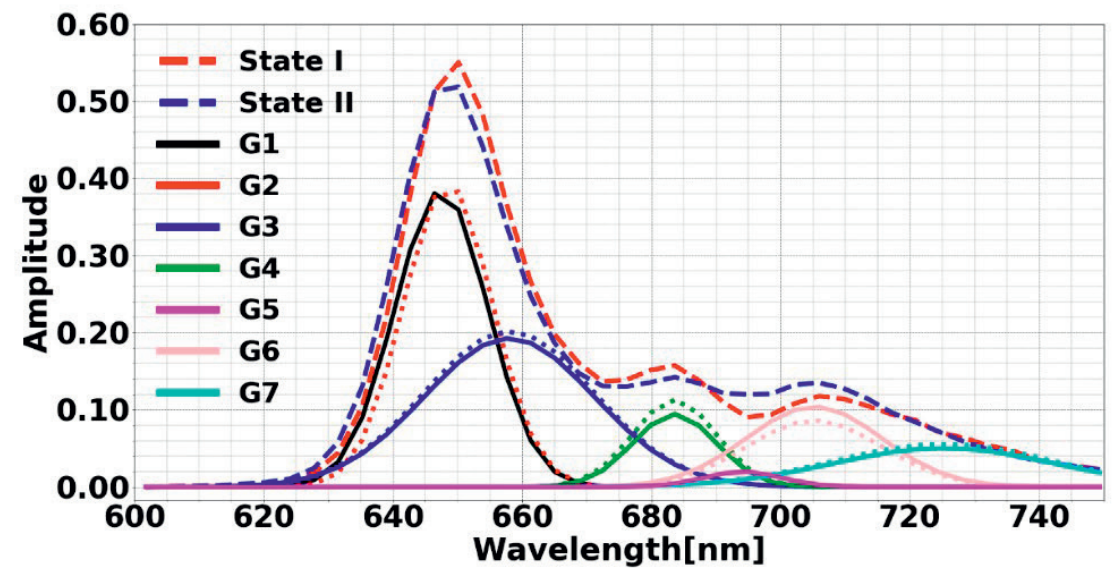

Figure S13. The Gaussian decomposition of the $4^{\text {th }}$ DAS (PBS excitation) of Synechococcus elongatus 7942 cells. The dotted (solid) Gaussians represent the Gaussians in state I (state II). The dashed red and blue curves show the DAS in state I and II from Figure 3D. Notice that G1 has a zero amplitude in state I while G2 has a zero amplitude in state II. G5 has a zero amplitude in state I.

In Table 4 the details of each Gaussian function in S13 are shown. The differences were calculated using equation 1 and 2. 
State transitions in the cyanobacterium Synechococcus elongatus 7942 involve reversible quenching of the photosystem II core

Table 4 The values describing the Gaussian functions in S13 and their changes in state I and state II.

\begin{tabular}{|c|c|c|c|c|}
\hline & $\begin{array}{l}\text { Position } \\
\text { in nm }\end{array}$ & $\begin{array}{l}\text { FWHM } \\
\text { in nm }\end{array}$ & $\begin{array}{l}\text { Percentage change in } \\
4^{\text {th }} \text { DAS (Figure 3D) }\end{array}$ & $\begin{array}{l}\text { Absolute change in } \\
4^{\text {th }} \text { DAS (Figure } \\
3 D \text { ) }\end{array}$ \\
\hline $\begin{array}{c}\text { Gaussian } \\
1\end{array}$ & 648 & 17.0 & ${ }^{*} \mathrm{a}$ & ${ }^{*} \mathrm{a}$ \\
\hline $\begin{array}{c}\text { Gaussian } \\
2\end{array}$ & 649 & 16.2 & ${ }^{*} \mathrm{a}$ & -0.30 \\
\hline $\begin{array}{c}\text { Gaussian } \\
3\end{array}$ & 658 & 31.0 & -5 & -0.30 \\
\hline $\begin{array}{c}\text { Gaussian } \\
4\end{array}$ & 684 & 15.2 & -17 & 0.48 \\
\hline $\begin{array}{c}\text { Gaussian } \\
5\end{array}$ & $694^{*} \mathrm{~b}$ & $14.0^{*} \mathrm{~b}$ & ${ }^{*} \mathrm{~b}$ & -0.23 \\
\hline $\begin{array}{c}\text { Gaussian } \\
6\end{array}$ & 705 & $24.7^{*} \mathrm{c}$ & 21 & -10 \\
\hline $\begin{array}{c}\text { Gaussian } \\
7\end{array}$ & 725 & $40.0^{*} \mathrm{~d}$ & & \\
\hline
\end{tabular}

*a In state I the amplitudes of G1 and G2 were zero and 6.77. In state II the amplitude of $\mathrm{G} 2$ became zero and that of $\mathrm{G} 1$ became 6.92 .

*b The position and FWHM of G5 were fixed to $694 \mathrm{~nm}$ and $14 \mathrm{~nm}$ during fitting. In state I G5 had a zero amplitude and in state II it had an amplitude of 0.30 .

${ }^{*} \mathrm{c}$ With respect to state II the amplitude of G6 decreased by $18 \%$ in state I as stated in the main text. G6 has amplitudes of 2.26 and 2.74 in state I and II, respectively.

${ }^{*} \mathrm{~d}$ The maximum allowed value for the FWHM of the Gaussian function during fitting was $40 \mathrm{~nm}$. 


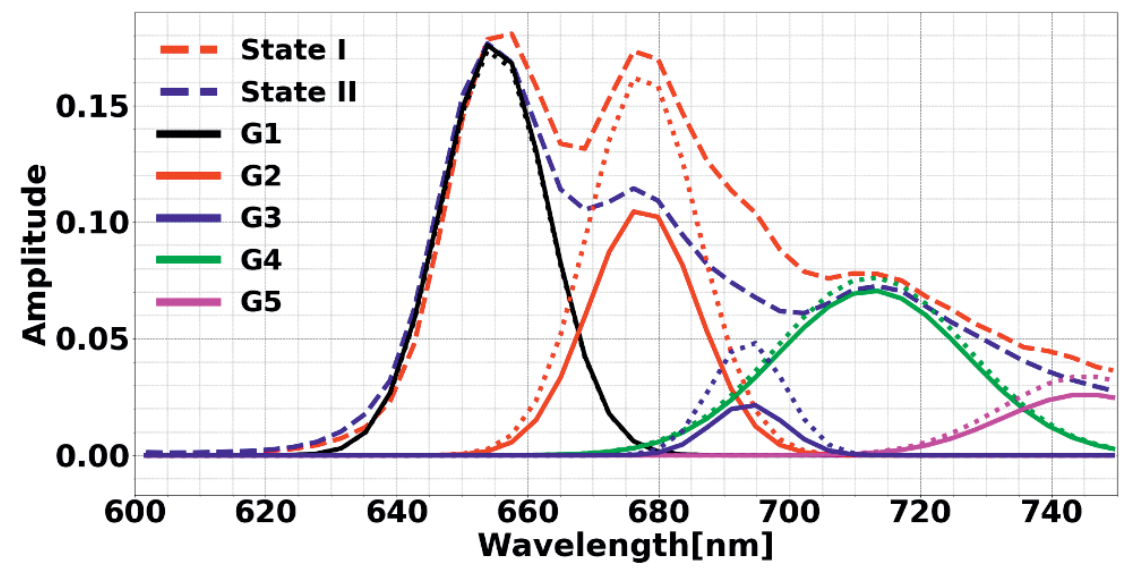

Figure S14. The Gaussian decomposition of the 5th DAS (PBS excitation) of Synechococcus elongatus 7942 cells. The dotted (solid) Gaussians represent the Gaussians in state I (state II). The dashed red and blue curves show the DAS in state I and II from Figure 3E.

In Table 5 the details of each Gaussian function in S14 are shown. The differences were calculated using Equations 1 and 2.

Table 5 The values describing the Gaussian functions in Figure S14 and their changes in state I and state II.

\begin{tabular}{|c|c|c|c|c|}
\hline & $\begin{array}{l}\text { Position } \\
\text { in nm }\end{array}$ & $\begin{array}{l}\text { FWHM } \\
\text { in nm }\end{array}$ & $\begin{array}{l}\text { Percentage change in } \\
\mathbf{5}^{\text {th }} \text { DAS (Figure 3E) }\end{array}$ & $\begin{array}{l}\text { Absolute change in } \\
\mathbf{5}^{\text {th }} \text { DAS (Figure } \\
\text { 3E) }\end{array}$ \\
\hline $\begin{array}{c}\text { Gaussian } \\
1\end{array}$ & 655 & 19.3 & 1 & 0.05 \\
\hline $\begin{array}{c}\text { Gaussian } \\
2\end{array}$ & 678 & 19.5 & -35 & -1.21 \\
\hline $\begin{array}{c}\text { Gaussian } \\
3\end{array}$ & 694 & 13.3 & -55 & -0.39 \\
\hline $\begin{array}{c}\text { Gaussian } \\
4\end{array}$ & 713 & 34.2 & -8 & -0.25 \\
\hline $\begin{array}{c}\text { Gaussian } \\
5\end{array}$ & 745 & 30.1 & -23 & -0.21 \\
\hline
\end{tabular}


State transitions in the cyanobacterium Synechococcus elongatus 7942 involve reversible quenching of the photosystem II core 


\section{4}

\section{Light harvesting dynamics in desert crust cyanobacteria: Changes in aggregation state as a mechanism for modulating energy transfer}

Leeat Bar Eyal, Reza Ranjbar Choubeh, Eyal Cohen, Ido Eisenberg, Carmen Tamburu, Márta Dorogi, Renata Ünnep, Marie-Sousai Appavou, Reinat Nevo, Uri Raviv, Ziv Reich, Győző Garab, Herbert van Amerongen, Yossi Paltiel, Nir Keren Proceedings of the National Academy of Sciences Aug 2017, 201708206; DOI: 10.1073/pnas. 1708206114 


\section{Abstract:}

In this paper we propose an energy dissipation mechanism that is completely reliant on changes in the aggregation state of the phycobilisome light harvesting antenna components. All photosynthetic organisms regulate the efficiency of excitation energy transfer (EET) to fit light energy supply to biochemical demands. Not many do this to the extent required of desert crust cyanobacteria. Following pre-dawn dew deposition, they harvest light energy with maximum efficiency until desiccating in the early morning hours. In the desiccated state absorbed energy is completely quenched.

Time and spectrally resolved fluorescence emission measurements of the desiccated desert crust Leptolyngbya ohadii strain identified (a) reduced EET between phycobilisome components (b) shorter fluorescence lifetimes (c) red shift in the emission spectra, compared to the hydrated state. These changes coincide with a loss of the ordered phycobilisome structure, evident from small angle neutron and X-ray scattering and cryo-TEM data.

Based on these observations we propose a model where in the hydrated state the organized rod structure of the phycobilisome supports directional EET to reaction centers with minimal losses due to thermal dissipation. In the desiccated state this structure is lost, giving way to more random aggregates. The resulting EET path will exhibit increased coupling to the environment and enhanced quenching.

\section{Significance statement}

All photosynthetic organisms regulate efficiency of excitation energy transfer (EET) to fit energy supply to biochemical demands. For desiccation tolerant desert crust cyanobacteria this ability is of the utmost importance. These organisms spend most of the daytime in the desiccated state whence absorbed energy is completely quenched. 
Light harvesting dynamics in desert crust cyanobacteria: Changes in aggregation state as a mechanism for modulating energy transfer

Based on our observations we propose a model where in the hydrated state the organized rod structure of the light-harvesting phycobilisome supports directional EET to reaction centers with minimal losses to thermal dissipation. In the desiccated state this structure gives way to more random aggregates. The resulting EET path exhibits increased coupling to the environment and enhanced quenching. This energy dissipation mechanism is completely reliant on changes in aggregation state of phycobilisome components.

\section{Introduction}

Deserts cover almost half of the Earth's terrestrial surface, and although desert conditions may seem unfavorable, they are home for diverse ecosystems. Many of these ecosystems are founded on biological desert crusts, which play an essential role in stabilizing shifting sands and enriching them with nutrients $(1,2)$. Cyanobacteria are among the first microorganisms to inhabit these crusts where one of the major sources of water is often dew deposited before dawn $(3,4)$. However, as temperatures elevate water quickly evaporates. Such conditions can be extremely harmful for photosynthetic organisms, and require adaptations on all cellular levels (39 ). These include shifts in metabolic profiles and the accumulation of compatible solutes. A key issue is the adaptation of the photosynthetic apparatus, since continued photosynthetic activity under high light, and especially in combination with desiccation may lead to the production of reactive oxygen species that will cause damage to the entire cell (10-12). The cyanobacteria that colonize sand crusts evolved strategies for coping with these daily cycles of hydration using mechanisms that enable extensive quenching of absorbed light energy. The extent of quenching in these organisms far exceeds that of common laboratory model organisms $(13,14)$. 
Our studies focused on Leptolyngbya ohadii, a crust cyanobacterium isolated from the Nizzana region of the NW Negev desert in Israel $(3,6)$. This is a keystone organism in this environment (4). To maintain L. ohadii cells in a viable state the desiccation process must be gradual (3). Recovery of photosynthetic activity, on the other hand, can occur immediately upon the addition of water (7). The desiccation/hydration transition does not require the break down and renewed production of photosynthetic protein complexes or pigments, and allows $L$. ohadii to utilize the short time of morning hydration for photosynthesis and avoid photo-damage during the remainder of the day (4).

A study of this process indicated similar absorption cross sections for desiccated and hydrated cyanobacteria i.e. L. ohadii absorbs a similar amount of light energy per wavelength when hydrated and when desiccated (7). Nevertheless, in the desiccated state electron transport is shut down and fluorescence yield decreases by $\sim 70 \%$. This requires efficient dissipation of excess energy to heat during desiccation together with a mechanism for fast energy distribution. We found evidence for a block in electron transport and dissipation of excitation energy through the accumulation of a stably oxidized $\mathrm{P}_{700}$ (primary photosystem I photochemical center). Oxidized chlorophylls are strong quenchers. Shrinkage of the lumenal space, observed in TEM, and restriction of plastocyanin diffusion provide a possible mechanism for the accumulation of $\mathrm{P}_{700^{+}}$. However, this is not the only protective mechanism at play in the desiccated state. Our data also indicated a strong quenching reaction in the phycobilisome (PBS) light-harvesting antenna (7).

PBSs are assembled by the association of phycobiliproteins (PBPs) to which linear tetrapyrroles are covalently linked. Typically, PBS cores contain cylinders made up of hexamers of the lowest energy absorbing PBP variant, allophycocyanin (APC; $\lambda_{\max }=650 \mathrm{~nm}$ ). PBS rods can be composed of a 
Light harvesting dynamics in desert crust cyanobacteria: Changes in aggregation state as a mechanism for modulating energy transfer

number of different PBPs (15). In the case of $L$. ohadii the rods contain phycocyanin (PC; $\left.\lambda_{\max }=620 \mathrm{~nm}\right)$.

In this study we dissected PBS fluorescence quenching processes in $L$. ohadii. We performed spectroscopic measurements on both hydrated and desiccated samples of $L$. ohadii, in order to uncover energy transfer pathways between components of the PBS system in the different states. Structural organization of the inter-thylakoidal space was examined by neutron and X-ray diffraction techniques. The results suggest a different excitation energy transfer (EET) path within the PBS antenna in hydrated and desiccated samples. The desiccated samples' EET path exhibits an efficient widespread quenching effect, resulting from interactions of PBS pigments.

\section{Results}

Under laboratory conditions $L$. ohadii is able to retain viability for prolonged periods and regain photosynthetic activity immediately after rehydration. We have demonstrated this in the past for electron transfer activity (7). Fig. 1 demonstrates this remarkable ability for PBS function. A desiccated culture, in which PBS fluorescence was strongly quenched, regained its high fluorescence yield as soon as 2 min after rehydration. The hydration/desiccation process was completely reversible. After $\sim 2 \mathrm{~h}$, at ambient temperature, the culture was desiccated and fluorescence was quenched. Rehydration resulted in immediate recovery (Fig. 1).

To understand the energy transfer dynamics controlling this process we conducted measurements using a streak-camera system that provides picosecond time resolution as well as spectral information. Measurements were performed on L. ohadii samples in the desiccated and the hydrated states. The excitation wavelength was set to $580 \mathrm{~nm}$, exciting mainly the PC 


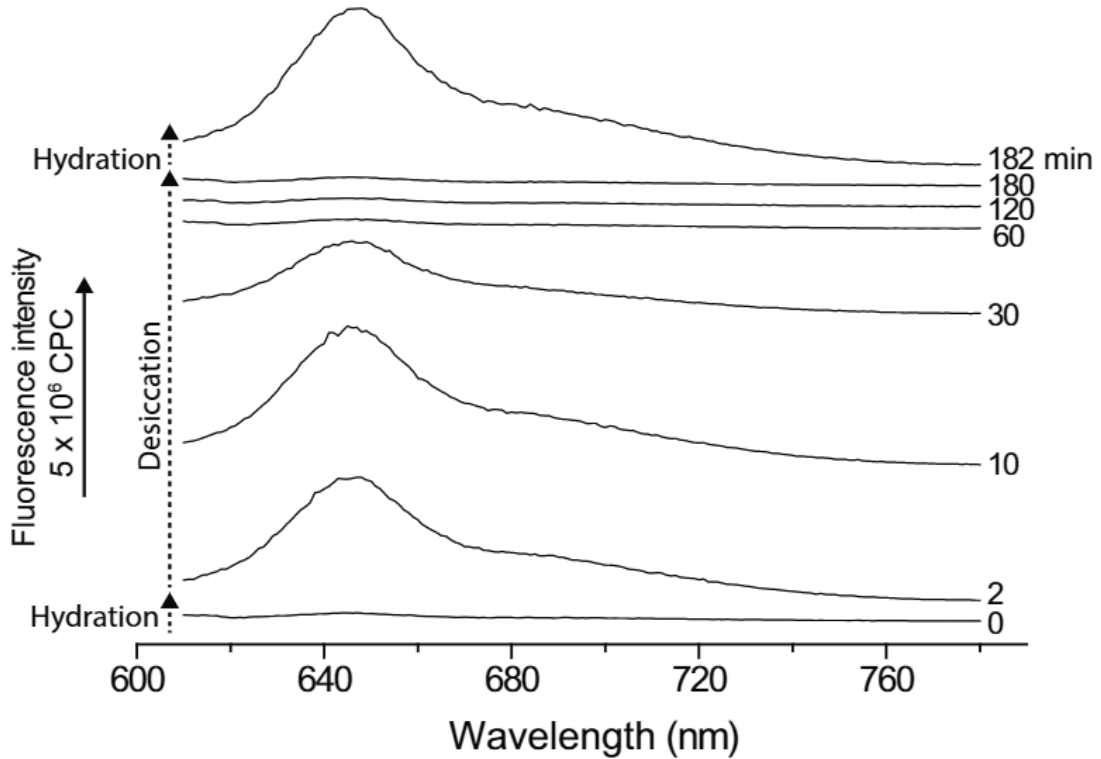

Figure 1: Reversibility of fluorescence yield during desiccation and rehydration. A desiccated $L$. ohadii culture was exposed to two cycles of desiccation and rehydration. Fluorescence spectra were measured with an excitation wavelength of $580 \pm 2 \mathrm{~nm}$. The traces are baseline shifted. The fluorescence trace of the desiccated sample did not exhibit any discernible peaks ( $0 \mathrm{~min}$ curve). However, immediately after rehydration PBS fluorescence was observed $(2 \mathrm{~min})$. The peak is dominated by PC fluorescence at $650 \mathrm{~nm}$ and contains a $670 \mathrm{~nm}$ APC shoulder. The filter was then desiccated for $180 \mathrm{~min}$ ( $2 \mathrm{~min}$ to $180 \mathrm{~min}$ ), before a second rehydration round. Again, an immediate recovery of PBS fluorescence was observed (182 $\mathrm{min}$ ).

component of the PBSs $(15,16)$. Fig. 2 presents an example of the raw data collected at room temperature $(A \& B)$ and at $77 \mathrm{~K}(C \& D)$ from hydrated and desiccated samples. Measurements conducted at room temperature best represent natural conditions. Measurements at $77 \mathrm{~K}$ allow better spectral resolution of the various fluorescence peaks. 
Light harvesting dynamics in desert crust cyanobacteria: Changes in aggregation state as a mechanism for modulating energy transfer

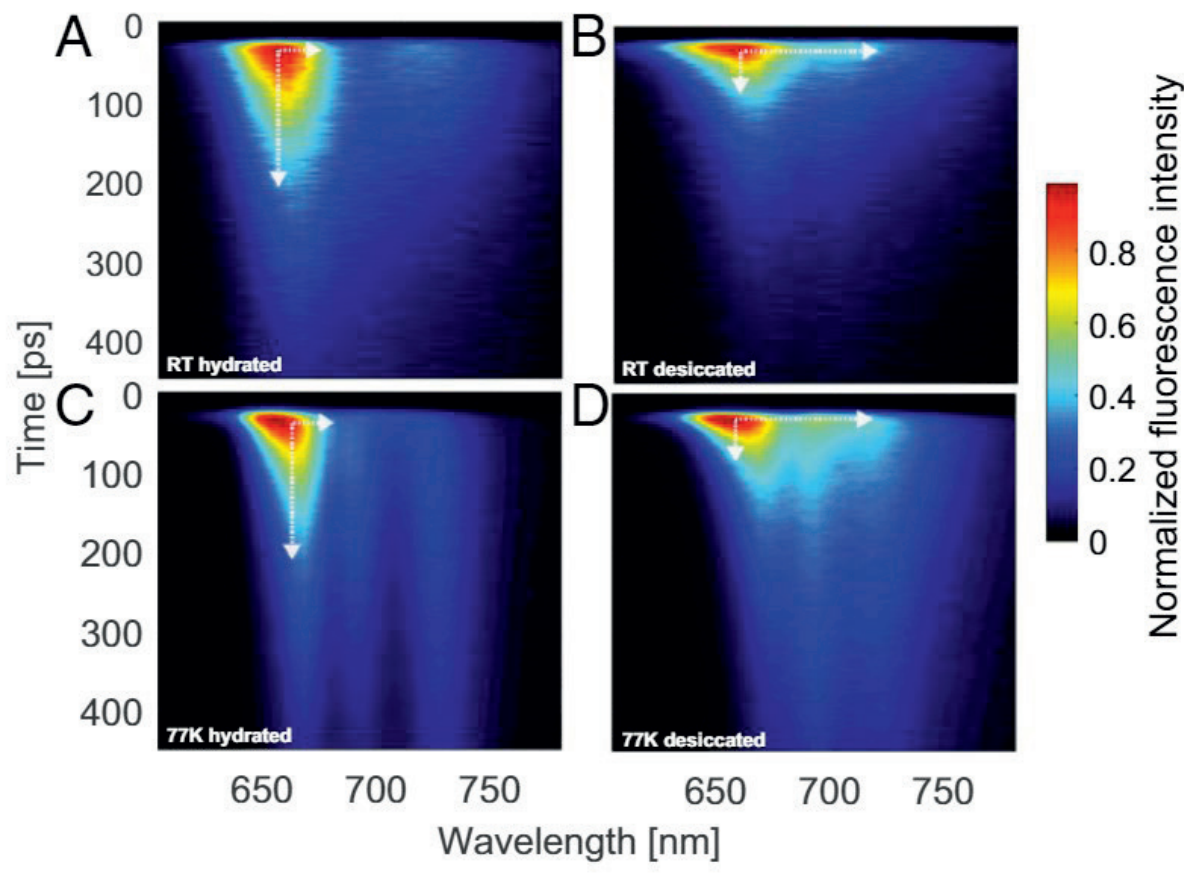

Figure 2: Propagation of photosynthetic fluorescence following $580 \mathrm{~nm}$ excitation with picosecond time resolution and spectral information. Panels $A$ and $B$ present data for room temperature measurements. Panels $C$ and $D$ present $77 \mathrm{~K}$ measurements. The fluorescence intensity was normalized (from 0 to 1 ), the scale is presented on the right hand side.

In the desiccated samples we observe a shortening of the lifetime and a redshift of the fluorescence, as compared to the hydrated sample. The differences are highlighted by white dashed arrows.

In the hydrated state at $77 \mathrm{~K}$, three spectral features emerge: $\sim 650-670 \mathrm{~nm}, \sim 670-700$ $\mathrm{nm}, \sim 720-750 \mathrm{~nm}$. These are normally ascribed to PBS, PSII and PSI fluorescence, respectively. However, we observed fluorescence at wavelengths $>700 \mathrm{~nm}$ in isolated L. ohadii PC (Fig. S2).

Several differences appear very clearly when comparing the raw room temperature data of the two states (highlighted by the arrows in Fig. 2). The first is the shortening of the desiccated sample's florescence lifetime across all wavelengths. The second prominent difference is the wavelength broadening of the desiccated sample fluorescence spectrum towards the 

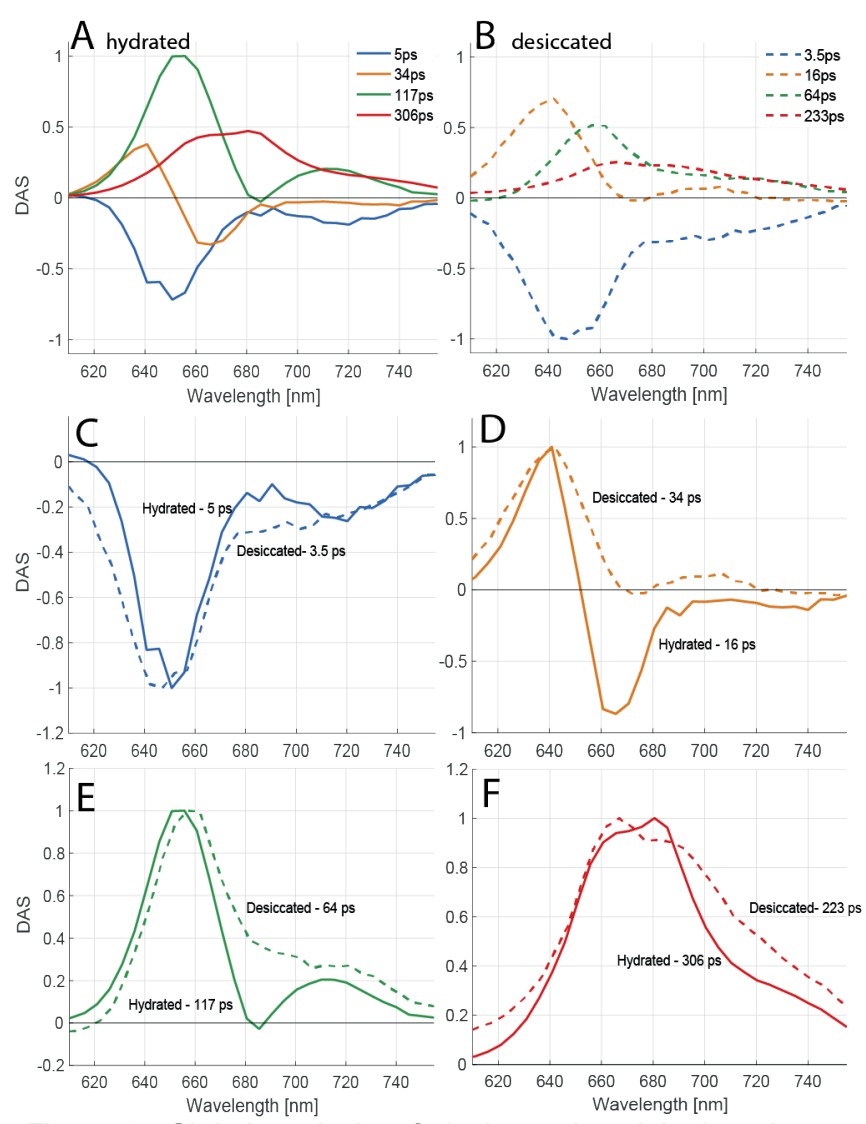

Figure 3: Global analysis of desiccated and hydrated states spectrally-resolved picosecond fluorescence. DAS and corresponding fluorescence lifetimes of hydrated (A) and desiccated (B) samples. Panels C-F present a comparison between the corresponding normalized DAS of hydrated and desiccated components. Each DAS is marked by its corresponding lifetime. The DAS of desiccated cells are dashed. A summary table for the observed lifetime ranges in 3-4 repeats is included in Table S1. In addition to DAS, the data was analyzed using a sequential model and the obtained EAS is presented in Fig. S1.

red, as compared to the hydrated sample. These observations are in line with the lower resolution Time-Correlated-Single-Photon-Counting (TCSPC) data in (7). The temporal and spectral resolution of the measurements reported 
Light harvesting dynamics in desert crust cyanobacteria: Changes in aggregation state as a mechanism for modulating energy transfer

here provide an opportunity to examine faster processes, occurring before thermal equilibration.

To resolve both spectral and temporal components, global analysis (17) was conducted on the data (Fig. 3; analysis by Evolution Associated Spectra (EAS) (17) is presented in Fig. S1). Table S1 summarizes the lifetimes of the various components. The best minimal fit for the data was obtained with four fluorescence decay lifetimes.

The 5 ps and 3.5 ps decay-associated spectra (DAS) of hydrated and desiccated samples respectively (Fig. $3 \mathrm{~A}$ and $3 \mathrm{~B}$ ) reflect excitation equilibration following absorption at $580 \mathrm{~nm}$. The $5 \mathrm{ps}$ DAS obtained for the hydrated sample is negative at all wavelengths. One of the peaks of this $5 \mathrm{ps}$ DAS is located at $650 \mathrm{~nm}$ and the other is located around 700-720 nm (Fig. $3 C)$. The lifetime $(\tau)$ and the DAS shape are rather similar to those observed in the laboratory model organism Synechocystis sp. PCC 6803 (16). In the desiccated sample the resolved lifetime of the above component is somewhat shorter (Table S1). The difference in the lifetimes of the 3.5 and 5 ps DAS is insignificant because the full width at half maximum (FWHM) of the instrument response function (IRF) is around 3.5-5 ps and this limits resolving a clear difference between these two short lifetimes. The shape of the 3.5 ps and 5 ps DAS of the desiccated and hydrated states show significant differences. In the desiccated state the $\sim 650 \mathrm{~nm}$ peak is broader and, instead of a distinct peak at $\sim 720 \mathrm{~nm}$, a broad shoulder extending from $680 \mathrm{~nm}$ to $740 \mathrm{~nm}$ is observed (Fig. 3).

In steady-state measurements, isolated PC from L. ohadii exhibited two peaks: one centered at $\sim 650 \mathrm{~nm}$ and another broader peak at $\sim 685 \mathrm{~nm}$. The latter extends well beyond $700 \mathrm{~nm}$ (Fig. S2). Similar steady-state spectra were observed in $\mathrm{PC}$ from other cyanobacterial species (see for example 
$(18,19)$ and many others). Based on the similarities with the in vitro data we attribute the first DAS component to PC EET. However, these are in vivo measurements and the possibility of emission from photosystem I (PSI) cannot be excluded. A significant contribution of PSI is, however, unlikely considering the $580 \mathrm{~nm}$ excitation wavelength.

The $34 \mathrm{ps}$ DAS in the hydrated state shows a positive peak at $\sim 640 \mathrm{~nm}$ and a negative peak at $\sim 660 \mathrm{~nm}$, indicating energy transfer from $\sim 640 \mathrm{~nm}$ to $\sim 660$ $\mathrm{nm}$ (Fig. 3D). This DAS was previously attributed, in a laboratory model cyanobacterium, to internal PBS energy transfer i.e. from PC to $P C$ and from $P C$ to $\operatorname{APC}_{660}(16,20)$. The corresponding DAS in the desiccated state has a lifetime of 16 ps (Fig. 3D), which is markedly different from the 34 ps DAS in the hydrated state. While the 34 ps DAS of the hydrated state shows EET, the 16 ps DAS of the desiccated state lacks a clear negative amplitude, indicating a major reduction of the corresponding EET process.

The 117 ps DAS in the hydrated state partly reflects EET from APC $_{660}$ to the terminal emitter $\mathrm{APC}_{680}$ or to chlorophylls fluorescing at $680 \mathrm{~nm}$ (16). The 64 ps DAS of the desiccated state lacks a negative peak indicating the absence of EET compared to the hydrated state. The EAS of this component show a prominent red shift and broadening of the desiccated state spectrum (Fig. S1).

The 306 ps DAS of the hydrated state and the 233 ps DAS of the desiccated state represent the disappearance of an equilibrated excitation distribution over all of the components of the system, namely PC, $\mathrm{APC}_{660}, \mathrm{APC}_{680}$, and chlorophylls. It is faster for the desiccated state and both DAS and EAS indicate a stronger contribution on the long-wavelength side.

The comparison between the desiccated and the hydrated fluorescence kinetics indicates (a) significantly reduced downhill EET (Fig. 3D and 3E) 
Light harvesting dynamics in desert crust cyanobacteria: Changes in aggregation state as a mechanism for modulating energy transfer

towards the RCs and (b) a substantial decrease of the fluorescence lifetimes, meaning that an additional non-radiative decay pathway has been created.

To test this further we performed measurements at $77 \mathrm{~K}$ (Fig. 2). A detailed description of the DAS decomposition is included in Fig. S3. At $77 \mathrm{~K}$ the fluorescence lifetimes of both hydrated and desiccated states are longer compared to room temperature. Furthermore, the differences between the desiccated and hydrated samples are smaller. Global analysis results indicate the convergence of both DAS shapes and lifetimes (Fig. S3). At 77 K PSI and photosystem II (PSII) fluorescence can be resolved. Nevertheless, in the desiccated samples the DAS decomposition indicates reduced EET from the PBSs to both photosystems.

The analysis of the measurements performed at room temperature and $77 \mathrm{~K}$ support the existence of a fast heat dissipation pathway in the desiccated state. This channel is apparently far more efficient at room temperature than at $77 \mathrm{~K}$. Quenching of excitation energy might be explained by the function of the orange carotenoid protein (OCP). OCP is photoactivated by high bluegreen irradiance, binds to APC and leads to the dissipation of excess energy absorbed in the PBSs under high-light conditions $(21,22)$. However, OCP is not a likely candidate for the non-radiative dissipation observed in the desiccated state. The extent of fluorescence quenching measured in desiccated L. ohadii is much larger (Fig. 1) than that induced by OCP in other cyanobacteria (e.g. $(16,22-24))$. Moreover, OCP binds and facilitates energy dissipation by interaction with the APC core (25). However, in the desiccated state we observed only little, if any, energy transfer from PC to APC. Furthermore, the overall effect of OCP knockout or over-expression on results of streak camera measurements is much smaller and focused to APC (16). 
We can consider two additional alternatives for the mechanism underlying the activity of the dissipation pathway. The first is a change within the structure of PBS pigment-protein complexes. Such a change can, for example, enhance charge transfer reactions between a bilin pigment and an adjacent amino acid, as demonstrated in the FMO (green sulfur bacteria antenna protein) and isiA (cyanobacterial iron stress induced antenna protein) chlorophyll binding proteins $(26,27)$. The second is a change in the aggregation state of the PBS proteins leading to the generation of intermediate-coupling interactions between them.

The visible absorption spectra of hydrated and desiccated samples did not show any significant difference in cross section or in line shape (7). Additional $C D$ measurements, in the visible range, failed to detect significant differences as well (Fig. S4). These data do not provide evidence for a major change in the energetic properties of the individual pigments in the hydrated and desiccated states. Furthermore, EPR spectroscopy did not detect a radical that can be associated with a charge transfer reaction in the desiccated state (7).

On the other hand, previous cryo-TEM studies of the ultrastructure of the inter-thylakoidal space harboring the PBSs did indicate differences between the two states (7). In the hydrated state the inter-thylakoidal space was well organized with uniform repeat distances and apparent rod structures traversing it. Such PBS architecture was also observed in Acaryochloris marina as well (28). Interestingly, both species effectively grow as biofilms. The well-organized architecture is lost in the desiccated state (an example of the TEM observations is included in Fig. S5). Additional ultrastructural information was gained through small angle $X$-ray and neutron scattering experiments (SAXS and SANS, Fig. 4). These techniques were used in a number of studies to measure the repeat distances (RDs) in different 
Light harvesting dynamics in desert crust cyanobacteria: Changes in aggregation state as a mechanism for modulating energy transfer
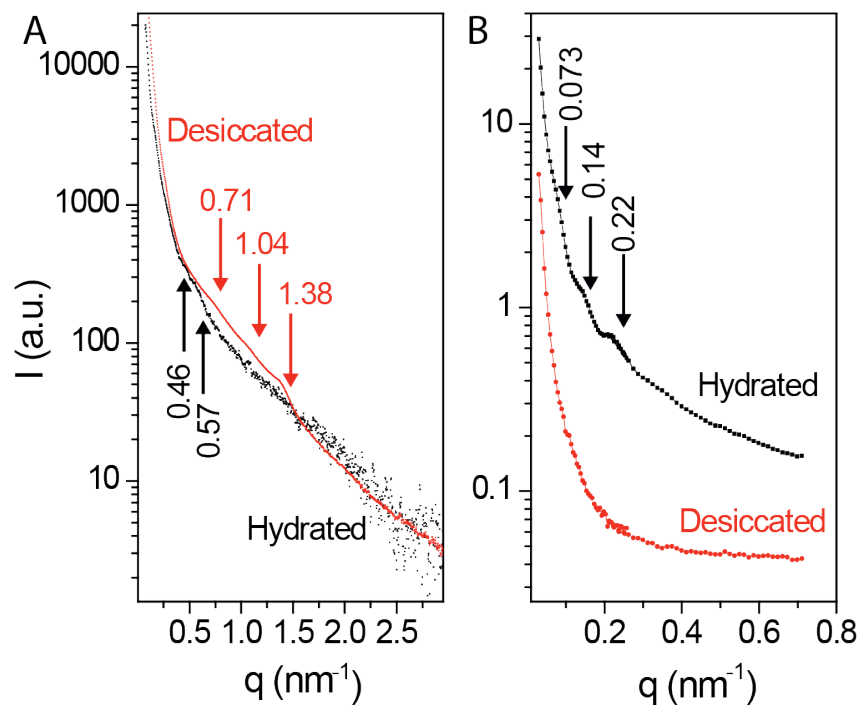

Figure 4: Small-angle $X$-ray and neutron scattering measurements. Hydrated and desiccated samples were studied by solution small-angle X-ray scattering (SAXS, A) and small-angle neutron scattering (SANS, B). The data presented were azimuthally integrated from the 2D raw data and plotted as intensity as a function of the magnitude of the scattering vector, $q$. In both cases, several weak intensity peaks were observed (indicated by arrows). On the right panel, azimuthally integrated SANS scattering curves were measured at $20 \mathrm{~m}$ (hydrated sample) and overlapped 20 $\mathrm{m}$ and $8 \mathrm{~m}$ (desiccated sample) sample-detector distances. The line represents the fitted curve (linear combination of a constant, and a power function and three Gaussians). No peak could be detected between 0.35 and $3.5 \mathrm{~nm}^{-1}$ on SANS curves. The first-order $q$ value of the features observed in the measurements can be translated into a repeat distance $R D=2 \pi / q$. Prominent features are marked directly on the graphs.

thylakoid membrane systems in vivo and in vitro (29-31). While providing 1D information, they do not require freezing or fixation.

Both SANS and SAXS scattering profiles carry information about key structural parameters, such as RDs of periodically organized multilamellar membrane systems, but with different sensitivities. The SAXS signal arises from differences in the electron density distribution while SANS is sensitive to protons. In the presence of $\mathrm{D}_{2} \mathrm{O}$, SANS allows distinguishing scattering length density between the aqueous regions and the lipid membrane (32). Also, SANS can usually measure in smaller $q$ ranges than SAXS. 
Accordingly, SAXS data provides information on relatively smaller intrathylakoidal RDs between electron densities defined by proteins and the lipid bilayer e.g. in the lumenal space, whereas SANS data provides information on the RD of thylakoid membranes.

In the hydrated sample SAXS features are observed at $q=0.46,0.57 \mathrm{~nm}^{-1}$ (Fig. 3A). These features are replaced by ones with higher $q$ values in the desiccated state $\left(0.71\right.$ and $1.04 \mathrm{~nm}^{-1} ; q=1.38 \mathrm{~nm}^{-1}$ might be the second harmonics of the principal peak at $\left.q=0.71 \mathrm{~nm}^{-1}\right)$. These $q$ values can be interpreted as decreased width of the thylakoid envelope in the desiccated state, which shrinks from 11-14 nm to 6-9 nm.

SANS data reported a feature at $q=0.072 \mathrm{~nm}^{-1}$ with two additional higher harmonic features at 0.139 and $0.221 \mathrm{~nm}^{-1}$. These values can be interpreted as the $R D \approx 87 \mathrm{~nm}$ of the inter-thylakoidal space. This is slightly larger than reported for Synechocystis 6803 (80 nm in (32) and $60 \mathrm{~nm}$ in (31)) and might indicate larger light harvesting antennae. In the desiccated state these characteristic features are almost completely lost. The scattering intensity decreased dramatically due to the loss of water. No Bragg peak could be discerned between 0.05 and $0.1 \mathrm{~nm}^{-1}$; instead, a broad shoulder emerged between 0.10 and $0.15 \mathrm{~nm}^{-1}$, which can be assigned to a broader range of inter-thylakoidal RDs.

The SAXS and SANS data fit well with cryo-TEM of hydrated and desiccated L. ohadii (Fig. S5, the complete analysis is in (7)). In the desiccated state, TEM images indicated a $~ 50 \%$ decrease in lumenal space, a value that is in line with the reduced RD observed in SAXS. This shrinkage was suggested to contribute to the PSI-based quenching mechanism $(7,33)$. They also indicated a loss of the organized thylakoid membrane space and of the apparent rod structures in the inter-thylakoidal space, which is in line with the 106 
Light harvesting dynamics in desert crust cyanobacteria: Changes in aggregation state as a mechanism for modulating energy transfer

SANS observations. Taken together cryo-TEM, SANS and SAXS support a change in the organization of the inter-thylakoidal space and in the aggregation state of the PBSs that populate it.

\section{Discussion}

In the desiccated state we observed (a) reduced EET between PBS components (b) extensive heat dissipation (c) fluorescence red shift (Fig. 23, Fig. S1-3). These changes coincide with a loss of the organized PBS structure in the inter-thylakoidal space (Fig. 4, Fig. S4-5).

How could a change in the aggregation state of PBSs support a transition between highly efficient EET and quenching? We can draw corollaries from in vitro studies in which we controlled the aggregation state of PC and APC artificially (34). Aggregation of PC in solution leads to shortening of their lifetime, red shift and a lower fluorescence yield. This was a reversible process that occurred in the absence of any additional auxiliary quenching proteins, such as OCP. The effect of aggregation on APC was much smaller.

It is important to note that aggregates discussed here are not chromophore aggregates but rather aggregates of chromophore-containing proteins. Unlike artificial cyanine and porphyrin aggregates, phycobilisomes represent a different scenario. Pigment distances within PC and APC subunits and between them dictate intermediate coupling strength $(35,36)$.

The underlying mechanism proposed is based on the intermediate-length (1$2 \mathrm{~nm}$ ) coupling interactions between PC units through the external $\beta 155$ pigments. A random network of intermediate coupling through the PC aggregates will generate long and convoluted EET paths and a band gap structure with many localized states that couple well to the wide frequency of environmental thermal "noise" $(37,38)$. In an intermediate coupling regime 
the band structure strongly depends on the level of disorder of the system, the splitting between bands is smaller and the gap is less protected. Such a structure will support decay to the lowest energy pigments together with quenching through an increased probability for interactions with the thermal bath. In APC, which lacks the external $\beta 155$ pigments, the chances for generating a network of intermediately coupled trimers are smaller and so are the effects of its aggregation state on EET.

$\mathrm{PC}$ is known to be sensitive to the ionic or dielectric properties of its environment (34). However, changes in these properties during desiccation are not expected to lead to the dramatic fluorescence quenching observed here (Fig. 1). Our data indicates small changes in the aggregation state with no detectable difference in the optical properties of the PC units themselves. We propose an in vivo model where in the hydrated state the organized rod structure supports directional EET to the RCs with minimal losses due to thermal dissipation (Fig. 5). In the desiccated state, this structure is lost, giving way to less organized aggregates. These less organized in vivo aggregates will exhibit EET properties similar to those of in vitro PC aggregates - with better coupling to wide frequency environmental noise (37).

This type of mechanism can also explain the temperature effect. In tightly coupled pigment networks (in Light Harvesting Complex II or FMO, for example, (39-42)) the energetic differences between $300 \mathrm{~K}$ and $77 \mathrm{~K}$ are too small to affect EET. Under an intermediate coupling regime, however, these temperature differences are expected to have a larger effect $(43,44)$. A mechanism based on small and localized changes in aggregation of PBS 
Light harvesting dynamics in desert crust cyanobacteria: Changes in aggregation state as a mechanism for modulating energy transfer

\section{Hydrated Maximum directional EET \\ Minimum heat dissipation}

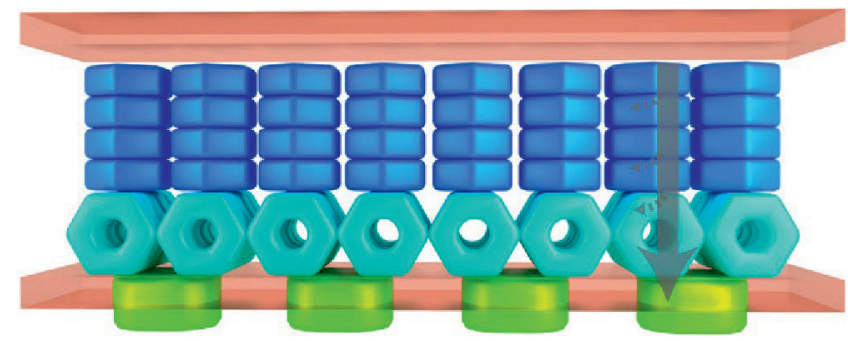

\section{Dessicated \\ Minimum directional EET \\ Maximum heat dissipation}

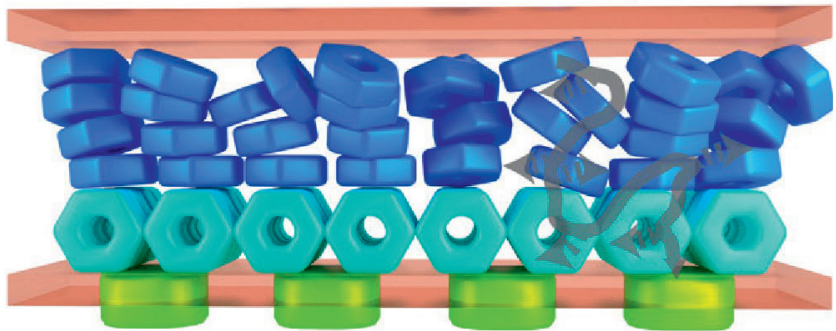

Figure 5: Model for EET processes in the hydrated and desiccated states. A schematic representation of the aggregation state of the PBS antenna in both states. The components are drawn roughly to scale. Green - photosystems, cyan - APC, blue - PC, gray arrows - directional EET, dashed arrows - heat dissipation. In the crowded inter-thylakoidal space only small changes in aggregation state are possible, these were exaggerated in the figure for demonstration purposes.

components in the crowded inter-thylakoidal space may explain the very fast rate of response to rehydration (Fig. 1).

The mechanism suggested here is akin to that proposed by Tamary and coworkers for high light induced quenching in Synechocystis 6803 where local heat transients generated in the PBSs by non-radiative energy dissipation were suggested to be the source of the non-photochemical quenching (NPQ) phenomenon (45). Furthermore, the idea that changes in the aggregation state can control the efficiency of EET in the photosynthetic antenna was considered as a mechanism for NPQ in plants. In vitro studies 
demonstrated that aggregation of Light Harvesting Complex II (LHCII) antenna complexes leads to efficient energy dissipation (46-51), although the significance of changes in LHCll aggregation state for energy dissipation in vivo is still debated $(52,53)$. It is important to note a number of key differences between LHCII and PC. (a) LHCII is trapped in the membrane. Its range of possible aggregation geometries is limited to the membrane plane. (b) The pigment-pigment distances within LHCII are, on average, shorter than within PC - supporting a tighter excitonic coupling regime. (c) LHCll contains carotenoids, dedicated to quenching. These are missing from PC. An aggregation based mechanism creating a network of intermediately coupled pigments and, henceforth, increased coupling to the thermal noise is much more likely in cyanobacteria than in vascular plant antennae.

Desiccation and rehydration in photosynthetic organisms are complex processes that require protection of many cellular processes, first and foremost, of the photosynthetic apparatus. The rate of recovery from desiccation is highly variable ranging from days in the case of certain vascular plants (54) to a few seconds in the case of desert crust cyanobacteria. So far, we were able to uncover two mechanisms for blocking photosynthetic activity in the desiccated state: constriction of the lumenal space leading to a block of plastocyanin diffusion, and accumulation of $\mathrm{P}_{700}{ }^{+}$ (7) and changes in the aggregation state of PBS components, as discussed here. In both cases the quenching mechanism relies on the rearrangement of existing components of the photosynthetic apparatus. In the new mechanism proposed here we also suggest a method for distributing the energy through a PC aggregate. It is the combination of all features of this desiccation mechanism that allows the very rapid response to rehydration.

Evidence for EET processes at the quantum/classical border are emerging from several studies of the photosynthetic apparatus and are highly relevant 
Light harvesting dynamics in desert crust cyanobacteria: Changes in aggregation state as a mechanism for modulating energy transfer

for new technologies. Here, small changes in configuration of an intermediately coupled pigment network supports a switch from highly efficient energy transfer to quenching (Fig. 5). In most cases, nano-scale technologies focus on energy transfer devices with tight coupling and low environmental noise (low temperatures) (55). The design principles of the desiccating PBSs suggest a new approach that will enable room temperature devices that can be switched to efficient energy transfer. By utilizing these principles it will be possible to develop a coupling controlled nano-toolbox for various future technologies including computers, materials and sensors.

\section{Materials and Methods}

\section{Culture growth and preparation for measurement}

Leptolyngbya ohadii cultures were grown (as described in (7)) in YBG11 medium in shaking flasks at $30{ }^{\circ} \mathrm{C}$ and $60 \mu$ mol photons $\mathrm{m}^{-2} \mathrm{~s}^{-1}$ provided by fluorescent lamps. Cells were homogenized and filtered on $0.45 \mu \mathrm{m}$ nitrocellulose (NC) filters. For measurement of dry samples, "artificial crust" filters were left to air dry at room temperature for at least two hours.

\section{Steady-state fluorescence measurements}

Samples were desiccated on the surface of a NC filter. The filter was mounted on a glass slide and fitted into the measurement chamber of a Fluoromax3 spectrofluorometer (Jobin Yvon). Hydration was performed in situ.

\section{Time-resolved fluorescence measurements}

Room temperature time-resolved fluorescence spectra were measured using a picosecond streak-camera set-up (56) as described in (16). The excitation wavelength was set to $580 \mathrm{~nm}$, in order to preferentially excite the PBSs. 77 $\mathrm{K}$ measurements were performed as described in (57). Measurements were 
made on either desiccated or hydrated filters of Leptolyngbya ohadii, and each measurement was repeated 2-4 times, measuring a different spot or sample each time. The samples were measured on NC filters at both room temperature and $77 \mathrm{~K}$. Global analysis (17) of the data was performed using the TIMP-based Glotaran program (58), in order to identify the spectral components in the fluorescence signal (see SI methods).

Solution Small angle $X$-ray and Neutron scattering

High-resolution small-angle X-ray scattering (SAXS) was performed as described in (59). Small-angle neutron scattering (SANS) was performed at the KWS-2 instrument operated by JCNS at the Heinz Maier-Leibnitz Zentrum (MLZ), Garching, Germany $((60,61)$ and SI methods). The scattering intensity, $I$, as a function of the magnitude of the momentum transfer vector, $q$, was then plotted and analyzed to determine the typical repeat distances (RDs) between membranes (62).

\section{Acknowledgments}

This work was supported by the ISF-UGC grant (2733/16) awarded to NK and the MOST-NSC grant awarded to NK and YP. RRC was supported by BioSolar Cells, co-financed by the Dutch Ministry of Economic Affairs and the FOM, part of The Netherlands Organization for Scientific Research (project 10TBSC24-3). GG and MD were supported by the National Research Development and Innovation Office of Hungary (OTKA K 112688 and GINOP-2.3.2-15-2016-00058 and PD 121243). 
Light harvesting dynamics in desert crust cyanobacteria: Changes in aggregation state as a mechanism for modulating energy transfer

\section{References}

1. Belnap J (2013) Microbiology. Some like it hot, some not. Science 340(6140):1533-4.

2. Garcia-Pichel F, Johnson SL, Youngkin D, Belnap J (2003) SmallScale Vertical Distribution of Bacterial Biomass and Diversity in Biological Soil Crusts from Arid Lands in the Colorado Plateau. Microb Ecol 46(3):312-321.

3. Raanan H, et al. (2016) Simulated soil crust conditions in a chamber system provide new insights on cyanobacterial acclimation to desiccation. Environ Microbiol 18(2):414-426.

4. Ohad I, Raanan H, Keren N, Tchernov D, Kaplan A (2010) Lightinduced changes within photosystem II protects Microcoleus sp. in biological desert sand crusts against excess light. PLoS One 5(6):e11000.

5. Ohad I, et al. (2005) Inactivation of photosynthetic electron flow during desiccation of desert biological sand crusts and Microcoleus sp.-enriched isolates. Photochem Photobiol Sci 4:977-982.

6. Harel Y, Ohad I, Kaplan A (2004) Activation of photosynthesis and resistance to photoinhibition in cyanobacteria within biological desert crust. Plant Physiol 136(2):3070-3079.

7. Bar-Eyal L, et al. (2015) An easily reversible structural change underlies mechanisms enabling desert crust cyanobacteria to survive desiccation. Biochim Biophys Acta - Bioenerg 1847(10):1267-1273.

8. Rajeev L, et al. (2013) Dynamic cyanobacterial response to hydration and dehydration in a desert biological soil crust. ISME J 
7(11):2178-2191.

9. Charuvi D, et al. (2015) Photoprotection conferred by changes in photosynthetic protein levels and organization during dehydration of a homoiochlorophyllous resurrection plant. Plant Physiol 167(4):1554-1565.

10. Kranner I, Birtic S (2005) A modulating role for antioxidants in desiccation tolerance. Integr Comp Biol 45:734-740.

11. Dinakar C, Bartels D (2013) Desiccation tolerance in resurrection plants: new insights from transcriptome, proteome and metabolome analysis. Front Plant Sci 4:482.

12. Barak S, Farrant JM (2016) Extremophyte adaptations to salt and water deficit stress. Funct Plant Biol 43(7):v-x.

13. Campbell D a, Hurry V, Clarke AK, Gustafsson P, Oquist G (1998) Chlorophyll fluorescence analysis of cyanobacterial photosynthesis and acclimation. Microbiol Mol Biol Rev 62(3):667-683.

14. Maxwell K, Johnson GN (2000) Chlorophyll fluorescence--a practical guide. J Exp Bot 51(345):659-668.

15. Adir N (2005) Elucidation of the molecular structures of components of the phycobilisome: Reconstructing a giant. Photosynth Res 85(1):15-32.

16. Tian L, et al. (2011) Site, rate, and mechanism of photoprotective quenching in cyanobacteria. J Am Chem Soc 133(45):18304-18311.

17. Van Stokkum IHM, Larsen DS, Van Grondelle R (2004) Global and target analysis of time-resolved spectra. Biochim Biophys Acta Bioenerg 1657(2-3):82-104. 
Light harvesting dynamics in desert crust cyanobacteria: Changes in aggregation state as a mechanism for modulating energy transfer

18. Debreczeny MP, Sauer K, Jianhui Z, Bryant DA (1993) Monomeric C-Phycocyanin at Room Temperature and $77 \mathrm{~K}$ : Resolution of the Absorption and Fluorescence Spectra of the Individual Chromophores and the Energy-Transfer Rate Constants. J Phys Chem 97:9852-9862.

19. Kupka M, Scheer H (2008) Unfolding of C-phycocyanin followed by loss of non-covalent chromophore-protein interactions. 1. Equilibrium experiments. Biochim Biophys Acta - Bioenerg 1777(1):94-103.

20. Tian L, et al. (2012) Picosecond kinetics of light harvesting and photoprotective quenching in wild-type and mutant phycobilisomes isolated from the cyanobacterium Synechocystis PCC 6803. Biophys J 102(7):1692-1700.

21. Kirilovsky D (2015) Modulating energy arriving at photochemical reaction centers: Orange carotenoid protein-related photoprotection and state transitions. Photosynth Res 126(1):3-17.

22. Kirilovsky D, et al. (2016) Cyanobacterial photoprotection by the orange carotenoid protein. Nat Plants 2(12):16180.

23. Boulay C, Wilson A, D'Haene S, Kirilovsky D (2010) Identification of a protein required for recovery of full antenna capacity in OCPrelated photoprotective mechanism in cyanobacteria. Proc Natl Acad Sci USA 107(25):11620-11625.

24. Wilson A, et al. (2006) A soluble carotenoid protein involved in phycobilisome-related energy dissipation in cyanobacteria. Plant Cell 18:992-1007.

25. Harris D, et al. (2016) Orange carotenoid protein burrows into the phycobilisome to provide photoprotection. Proc Natl Acad Sci USA 
113(12):E1655-62.

26. Rolczynski BS, Navotnaya P, Sussman HR, Engel GS (2016) Cysteine-mediated mechanism disrupts energy transfer to prevent photooxidation. Proc Natl Acad Sci USA 113(31):8562-8564.

27. Chen H-YS, Liberton M, Pakrasi HB, Niedzwiedzki DM (2017) Reevaluating the Mechanism of Excitation Energy Regulation in IronStarved Cyanobacteria. Biochim Biophys Acta-Bioenerg.

28. Chen M, Floetenmeyer M, Bibby TS (2009) Supramolecular organization of phycobiliproteins in the chlorophyll d-containing cyanobacterium Acaryochloris marina. FEBS Lett 583(15):2535-9.

29. Ünnep R, et al. (2014) The ultrastructure and flexibility of thylakoid membranes in leaves and isolated chloroplasts as revealed by smallangle neutron scattering. Biochim Biophys Acta-Bioenerg 1837(9):1572-1580.

30. Nagy G, et al. (2011) Reversible membrane reorganizations during photosynthesis in vivo: revealed by small-angle neutron scattering. Biochem J 436:225-230.

31. Liberton M, et al. (2013) Organization and flexibility of cyanobacterial thylakoid membranes examined by neutron scattering. J Biol Chem 288(5):3632-40.

32. Unnep R, Nagy G, Markó M, Garab G (2014) Monitoring thylakoid ultrastructural changes in vivo using small-angle neutron scattering. Plant Physiol Biochem 81:1-11.

33. Kirchhoff H, et al. (2011) Dynamic control of protein diffusion within the granal thylakoid lumen. Proc Natl Acad Sci 108(50):2024820253. 
Light harvesting dynamics in desert crust cyanobacteria: Changes in aggregation state as a mechanism for modulating energy transfer

34. Eisenberg I, et al. (2017) Regulating the Energy Flow in a Cyanobacterial Light Harvesting Antenna Complex. J Phys Chem B.

35. Kobayashi T (2012) J-Aggregates (World Scientific Publisinng, Singapure).

36. Clark J, Silva C, Friend RH, Spano FC (2007) Role of intermolecular coupling in the photophysics of disordered organic semiconductors: Aggregate emission in regioregular polythiophene. Phys Rev Lett 98(20):1-4.

37. Chin a. W, et al. (2013) The role of non-equilibrium vibrational structures in electronic coherence and recoherence in pigmentprotein complexes. Nat Phys 9(2):113-118.

38. Rebentrost P, Mohseni M, Kassal I, Lloyd S, Aspuru-Guzik A (2009) Environment-assisted quantum transport. New J Phys 11. doi:10.1088/1367-2630/11/3/033003.

39. Scholes GD (2010) Quantum-coherent electronic energy transfer: Did nature think of it first? J Phys Chem Lett 1(1):2-8.

40. Ishizaki A, Fleming GR (2009) Theoretical examination of quantum coherence in a photosynthetic system at physiological temperature. Proc Natl Acad Sci USA 106(41):17255-17260.

41. van Amerongen $H$, van Grondelle R (2001) Understanding the Energy Transfer Function of LHCII, the Major Light-Harvesting Complex of Green Plants. J Phys Chem B 105(3):604-617.

42. Gradinaru CC, et al. (1998) The flow of excitation energy in LHCll monomers: implications for the structural model of the major plant antenna. Biophys J 75(6):3064-77. 
43. Moix JM, Khasin M, Cao J (2013) Coherent quantum transport in disordered systems: I. the influence of dephasing on the transport properties and absorption spectra on one-dimensional systems. New J Phys 15. doi:10.1088/1367-2630/15/8/085010.

44. Zhong X, Zhao Y, Cao J (2014) Coherent quantum transport in disordered systems: II. Temperature dependence of carrier diffusion coefficients from the time-dependent wavepacket diffusion method. New J Phys 16. doi:10.1088/1367-2630/16/4/045009.

45. Tamary E, et al. (2012) Structural and functional alterations of cyanobacterial phycobilisomes induced by high-light stress. Biochim Biophys Acta 1817:319-327.

46. Johnson MP, et al. (2011) Photoprotective energy dissipation involves the reorganization of photosystem II light-harvesting complexes in the grana membranes of spinach chloroplasts. Plant Cell 23:1468-1479.

47. Horton P, Wentworth M, Ruban A (2005) Control of the light harvesting function of chloroplast membranes: The LHCIIaggregation model for non-photochemical quenching. FEBS Lett 579(20):4201-4206.

48. Bassi R, Silvestri M, Dainese P, Moya I, Giacometti GM (1991) Effects of a non-ionic detergent on the spectral properties and aggregation state of the light-harvesting chlorophyll a/b protein complex (LHCII). J Photochem Photobiol B Biol 9(3-4):335-353.

49. Barzda V, Garab G, Gulbinas V, Valkunas L (1996) Evidence for long-range excitation energy migration in macroaggregates of the chlorophyll a/b light-harvesting antenna complexes. Biochim Biophys Acta-Bioenerg 1273(3):231-236. 
Light harvesting dynamics in desert crust cyanobacteria: Changes in aggregation state as a mechanism for modulating energy transfer

50. Chmeliov J, et al. (2016) The nature of self-regulation in photosynthetic light-harvesting antenna. Nat Plants 2:16045.

51. Kirchhoff H, Hinz HJ, Rösgen J (2003) Aggregation and fluorescence quenching of chlorophyll a of the light-harvesting complex II from spinach in vitro. Biochim Biophys Acta-Bioenerg 1606(1-3):105-116.

52. Dinc E, et al. (2016) LHCSR1 induces a fast and reversible pHdependent fluorescence quenching in LHCII in Chlamydomonas reinhardtii cells. Proc Natl Acad Sci USA 7673-7678.

53. Ruban A V., et al. (2007) Identification of a mechanism of photoprotective energy dissipation in higher plants. Nature 450(7169):575-578.

54. Vicré M, Farrant JM, Driouich A (2004) Insights into the cellular mechanisms of desiccation tolerance among angiosperm resurrection plant species. Plant, Cell Environ 27(11):1329-1340.

55. Clarke J, Wilhelm FK (2008) Superconducting quantum bits. Nature 453:1031-1042.

56. van Stokkum IHM, van Oort B, van Mourik F, Gobets B, van Amerongen $\mathrm{H}$ (2008) (Sub)-picosecond spectral evolution of fluorescence studied with a synchroscan streak-camera system and target analysis. Biophysical Techniques in Photosynthesis, eds Aartsma TJ, Matysik J (Springer Netherlands), pp 223-240.

57. Chukhutsina V, Bersanini L, Aro E-M, van Amerongen H (2015) Cyanobacterial Light-Harvesting Phycobilisomes Uncouple From Photosystem I During Dark-To-Light Transitions. Sci Rep 5:14193.

58. Snellenburg JJ, Laptenok SP, Seger R, Mullen KM, van Stokkum 
IHM (2012) Glotaran : A Java -Based Graphical User Interface for the R Package TIMP. J Stat Softw 49(3).

59. Nadler M, et al. (2011) Following the structural changes during zincinduced crystallization of charged membranes using time-resolved solution X-ray scattering. Soft Matter 7(4):1512.

60. Nagy G, et al. (2014) Chloroplast remodeling during state transitions in Chlamydomonas reinhardtii as revealed by noninvasive techniques in vivo. Proc Natl Acad Sci USA 111(13):5042-7.

61. Radulescu A, et al. (2016) Studying Soft-matter and Biological Systems over a Wide Length-scale from Nanometer and Micrometer Sizes at the Small-angle Neutron Diffractometer KWS-2. J Vis Exp (118):1-23.

62. Steiner A, et al. (2012) Entropic attraction condenses like-charged interfaces composed of self-assembled molecules. Langmuir 28:2604-2613.

63. Gussakovsky, E. E., Barzda, V., Shahak, Y., \& Garab, G. (1997). Irreversible disassembly of chiral macrodomains in thylakoids due to photoinhibition. Photosynth Res, 51(2), 119-126. 
Light harvesting dynamics in desert crust cyanobacteria: Changes in aggregation state as a mechanism for modulating energy transfer

\section{Supporting Information}

\section{Time-Resolved Fluorescence Measurements and Global Analysis}

A time window of either 800 or 2,000 ps was used for the timeresolved fluorescence measurements. The repetition rate of the laser was $4 \mathrm{MHz}$. The intensity of the 580$\mathrm{nm}$ excitation light was $\sim 100 \mathrm{nW}$.

The IRF was modeled as a single Gaussian function. The timeresolved spectra were fitted as a superposition of four exponentially decaying components, in the form $S(t, \lambda)=\sum_{i=1}^{n} c_{i}(t) \varepsilon_{i}(\lambda)$.

The resulting DAS components are presented after reduction of the contribution of the IRF and time 0 correction. For a sequential model, in which each component decays to the next one, EAS components were obtained (Fig. S1).

\section{Solution Small-Angle Neutron Scattering}

A wavelength of $5.15 \AA$ was used as well as a sample to detector of 2,8 , and $20 \mathrm{~m}$ to cover a q range from 0.03 to $3.6 \mathrm{~nm}^{-1}$. The samples were held in 2-mm-thick quartz cells. The reduction of raw data was performed by the routine qtiKWS (iffwww.iff. kfajuelich.de/ pipich/dokuwiki/doku.php/qtikws) including corrections for detector sensitivity, background noise and empty cell signal. Absolute determination of scattering cross sections $\mathrm{I}(\mathrm{q})=\mathrm{d} \Sigma / \mathrm{d} \Omega$ per unit sample volume in $\mathrm{cm}^{-1}$ was obtained thanks to a calibration with a $1.5-\mathrm{mm}$-thick polymethylmethacrylate sample. In both cases the resulting 2D scattering patterns were azimuthally integrated (average intensities belonging to identical q values). 

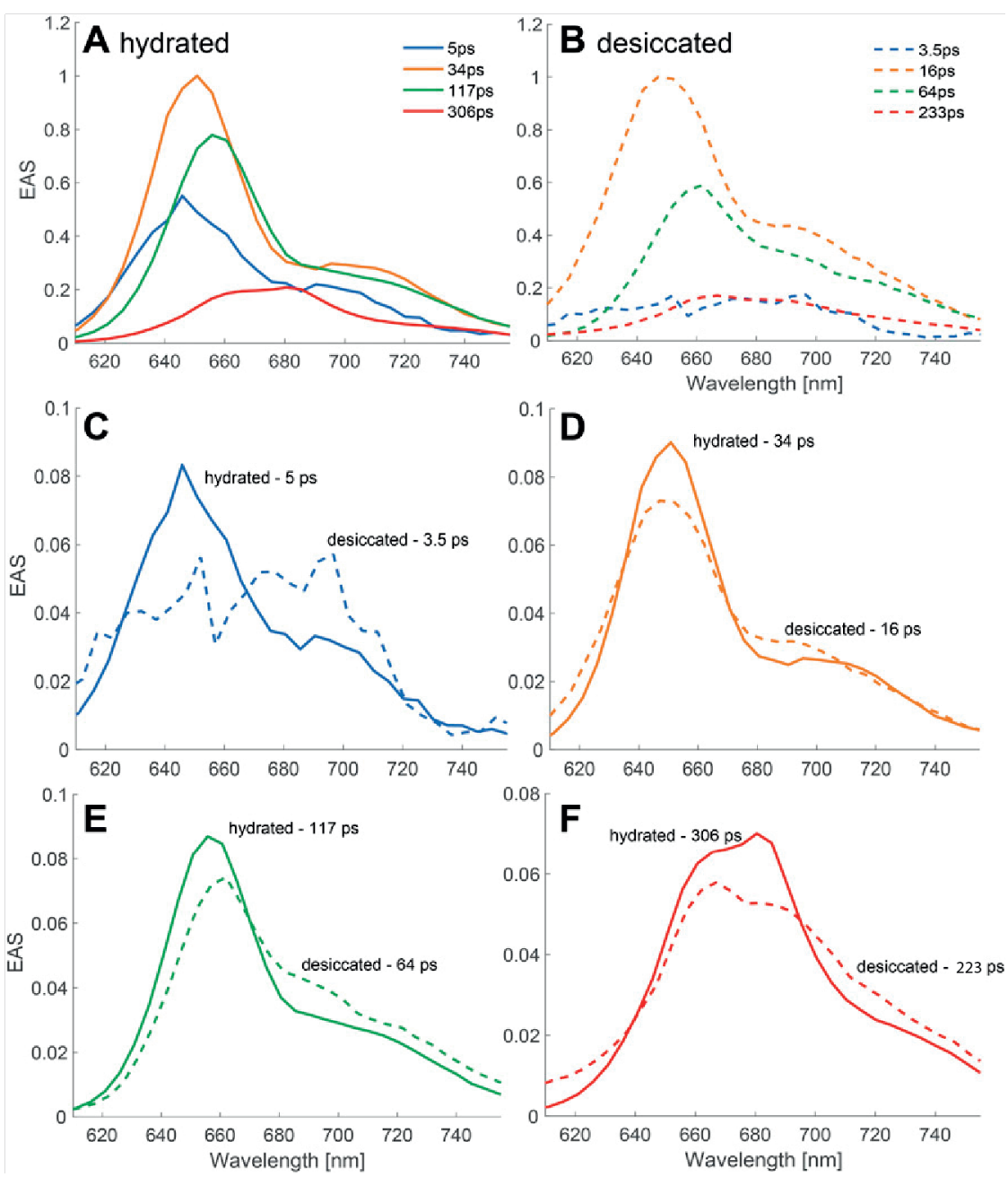

Fig. S1. EAS analysis of spectrally resolved picosecond fluorescence at RT. EAS and corresponding fluorescence lifetimes of (A) hydrated and (B) desiccated samples. (C-F) Comparison between the corresponding hydrated and desiccated EAS components. 
Light harvesting dynamics in desert crust cyanobacteria: Changes in aggregation state as a mechanism for modulating energy transfer
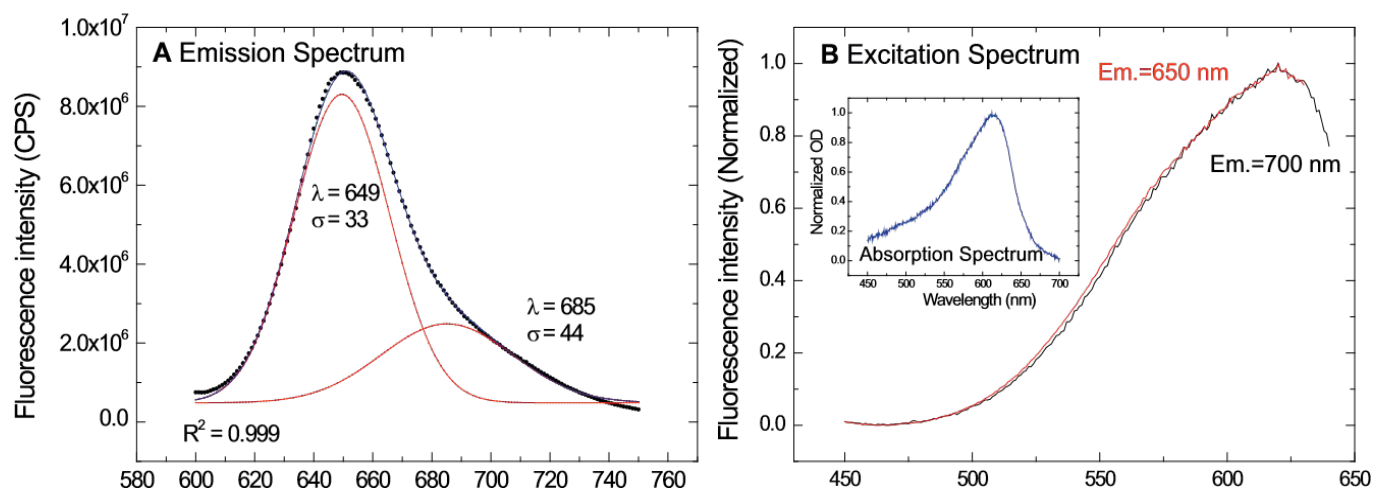

Fig. S2. Steady state fluorescence spectra of phycocyanin isolated from Leptolyngbya ohadii. (A) The emission spectrum (blue) was measured following excitation at $580 \mathrm{~nm}$, whereas the red Gaussian fitted to the spectrum shows two emission peaks: one at $649 \mathrm{~nm}$ and another at $685 \mathrm{~nm}$. (B) The excitation spectra of the 650 - and $700-\mathrm{nm}$ emission bands. Inset represents the corresponding absorption spectra of the sample. 

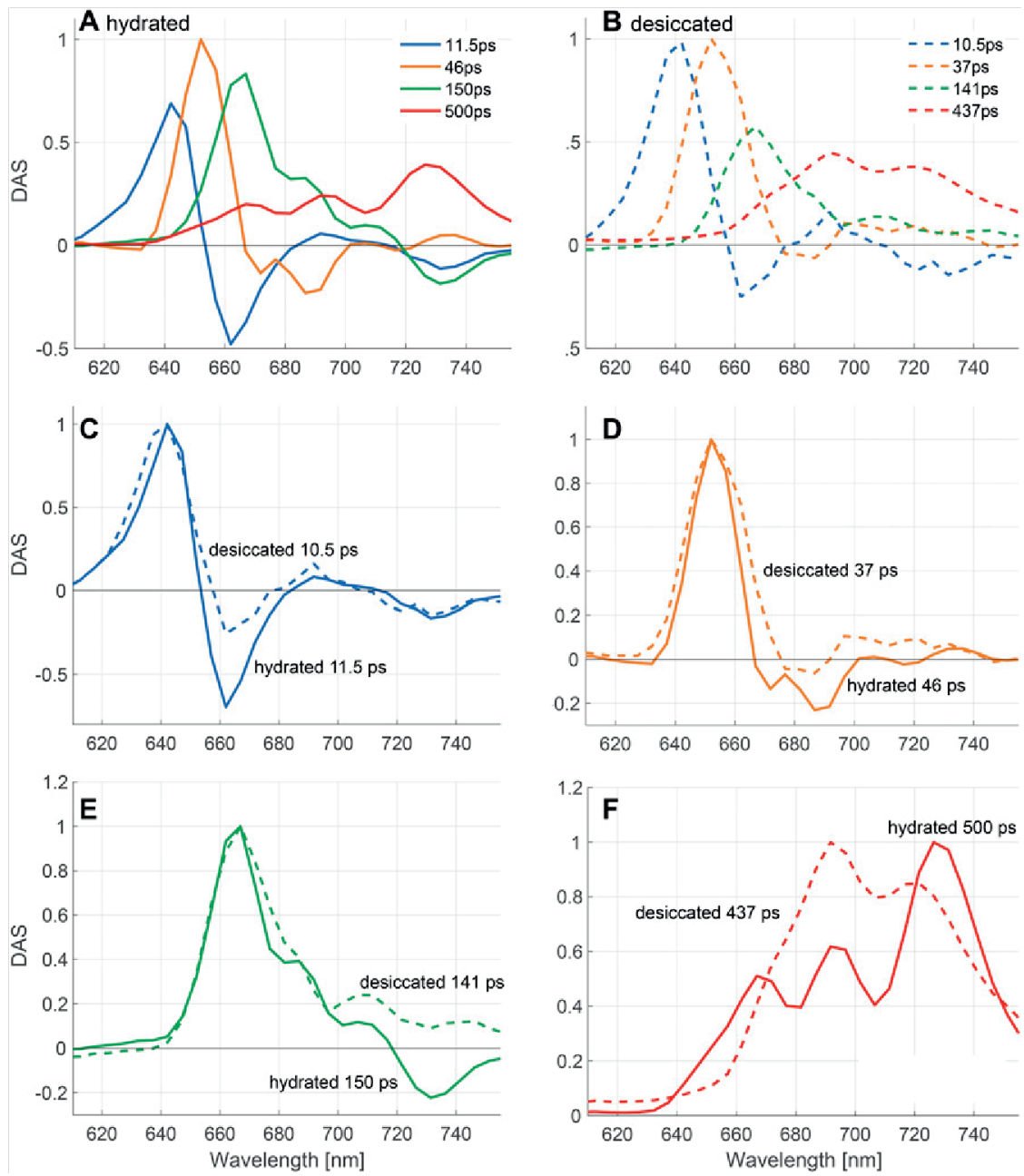

Fig. S3. DAS decomposition of 77-K measurements. DAS and corresponding fluorescence lifetimes of (A) hydrated and (B) desiccated samples. (C-F) Comparison between the corresponding hydrated and desiccated normalized DAS components. At $77 \mathrm{~K}$ the contribution of PSII, PSI, and PBS components can be better resolved spectrally (5). Both the 11.5-ps DAS of the hydrated state and the 10.5-ps DAS of the desiccated state indicate EET from 640 to $660 \mathrm{~nm}$. They also show EET from 640 or 690 to $730 \mathrm{~nm}$. This wavelength range corresponds to PSI fluorescence in the steady-state spectra (5). Based on the lower intensity of the negative peak at $\sim 660 \mathrm{~nm}$, the relative proportion of the PBS EET process is smaller in the desiccated sample. The 46-ps DAS of the hydrated state and the 36-ps DAS of the desiccated state have a positive peak at $650 \mathrm{~nm}$ accompanied by a negative feature at $670-690 \mathrm{~nm}$ that can be attributed to EET to APC 680 and PSII. This feature is smaller in the desiccated state and not as well resolved. The 150-ps DAS of the hydrated state and the 141-ps DAS of the desiccated state look similar between 610 and $700 \mathrm{~nm}$, 
Light harvesting dynamics in desert crust cyanobacteria: Changes in aggregation state as a mechanism for modulating energy transfer

but they are different around $730 \mathrm{~nm}$, where a negative peak appears only in the hydrated sample. We can speculate that the initial fast EET to $730 \mathrm{~nm}$ observed in the 11.5-ps DAS of the hydrated

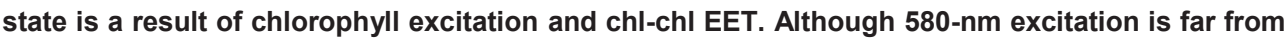
the chlorophyll absorption peak, the extinction coefficient at this wavelength is, nevertheless, not zero. The EET pathway from PC to PSI is longer and observed only in the 150-ps DAS of the hydrated state. This transfer is blocked in the desiccated state. The 500-ps DAS of the hydrated state exhibits three distinct peaks that can be associated with longer decay times of PBSs, PSII, and PSI. The 660-nm PBS peak, which is prominent in the 500-ps DAS of the hydrated state, is nearly absent in the 437-ps DAS of the desiccated state.

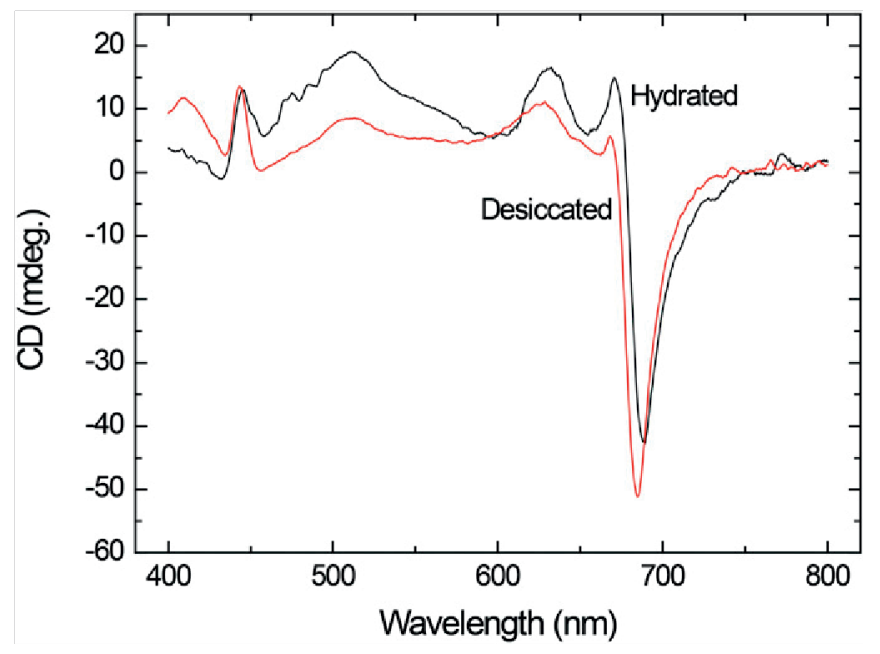

Fig. S4. CD spectra. CD spectra of hydrated and desiccated samples (as indicated in the graph). The data are averaged over 4-20 repeats, baseline subtracted, and normalized to the absorption of the red chlorophyll peak. The most characteristic peaks include the chlorophyll-associated peaks at $\sim 445$ and the split band in the red with the positive counterpart. In cyanobacteria these are predominantly associated with PSI. The PBS peak is at $\sim 620$, and the $\sim 510-\mathrm{nm}$ band represents a carotenoid signal. All of these features can be observed both in the hydrated and in the desiccated sample. The experiment was performed as described in ref. 63. 

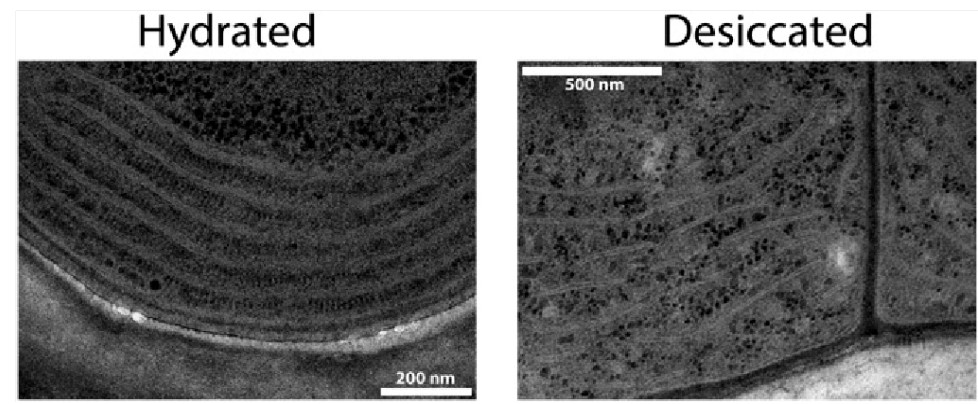

Fig. S5. Examples of TEM images of hydrated and desiccated samples. The repeated structures in the space between thylakoid envelopes that are clearly evident in the hydrated state are lost in the desiccated state (for methods and a thorough analysis of the TEM data, see ref. 7).

Table S1.Global analysis lifetimes

\begin{tabular}{|c|c|c|}
\hline Component & Desiccated [average (range)] & $\begin{array}{c}\text { Hydrated [average } \\
\text { (range) }\end{array}$ \\
\hline Room temperature & & 4 \\
\hline $\mathrm{n}$ & 3 & $5(4.5-5.5) \mathrm{ps}$ \\
\hline First & $4(3.2-4) \mathrm{ps}$ & $34(30-37) \mathrm{ps}$ \\
\hline Second & $16(15-18) \mathrm{ps}$ & $117(111-128) \mathrm{ps}$ \\
\hline Third & $64(60-66) \mathrm{ps}$ & $306(294-315) \mathrm{ps}$ \\
\hline Fourth & $233(221-240) \mathrm{ps}$ & 3 \\
\hline $\mathrm{n} \mathrm{K}$ & 2 & $12(10.5-12.5) \mathrm{ps}$ \\
\hline First & $10(10-10.5) \mathrm{ps}$ & $45(41-47.5) \mathrm{ps}$ \\
\hline Second & $37(37-37) \mathrm{ps}$ & $143(130-152) \mathrm{ps}$ \\
\hline Third & $127(113-141) \mathrm{ps}$ & $498(490-510) \mathrm{ps}$ \\
\hline Fourth & $443(437-449) \mathrm{ps}$ & \\
\hline
\end{tabular}

Lifetimes of DAS components presented in Fig. 2. The average lifetime and the range between the maximum and minimum values recorded for the biological repeats are presented. 
Light harvesting dynamics in desert crust cyanobacteria: Changes in aggregation state as a mechanism for modulating energy transfer 


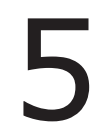

Efficiency of excitation-energy trapping in the reaction center of the low-light adapted green photosynthetic bacterium Chlorobaculum tepidum

Reza Ranjbar Choubeh, Rob B.M. Koehorst, David Bína, Paul C. Struik, Jakub Pšenčík, Herbert van Amerongen 


\section{Chapter 5}

\section{Abstract:}

During the millions of years of evolution, photosynthetic organisms have adapted to almost all terrestrial and aquatic habitats, although some environments are obviously more suitable for photosynthesis than others. For the description of the effectiveness of photosynthesis, the quantum efficiency of photochemistry plays an important role. The quantum efficiency covers all the processes from the photon absorption in lightharvesting complexes up to the charge separation in the reaction centers. Photosynthetic organisms living in low-light conditions require on the one hand a large light-harvesting apparatus to absorb as many photons as possible. On the other hand, the excitation trapping time scales with the size of the light-harvesting system, and the longer the distance over which the formed excitations have to be transferred, the larger the probability to lose excitations. Therefore a compromise between photon capture efficiency and excitation trapping efficiency needs to be found. Here we report results on the efficiency of the excitation energy transfer and charge separation in whole cells of the green sulfur bacterium Chlorobaculum tepidum. This organism is able to live in environments with very low illumination. The efficiency of its photochemistry is investigated. Using fluorescence measurements with picosecond resolution, we estimate that despite a rather large size and complex composition of its light-harvesting apparatus, the efficiency of its photochemistry is around $85-90 \%$ at $20^{\circ} \mathrm{C}, 80-86 \%$ at $45^{\circ} \mathrm{C}$, and about $81-90 \%$ at $77 \mathrm{~K}$ when part of the excitation energy is trapped on low-energy $\mathrm{BChl}$ a molecules. The data are evaluated using target analysis, which provides further insight into the functional organization of the low-light adapted photosynthetic apparatus. 
Efficiency of excitation-energy trapping in the reaction center of the low-light adapted green photosynthetic bacterium Chlorobaculum tepidum

\section{Introduction}

Photosynthesis provides energy for the majority of organisms on Earth. The process starts in light-harvesting (LH) complexes that absorb light and transfer the excitation energy to reaction centers (RCs). In these RCs charge separation (CS) occurs, which drives chemical reactions that ultimately result in storage of light energy in a chemical form. While all RCs are structurally similar, the need to adapt to different light conditions has led to the evolution of a broad diversity of LH complexes (Huner et al. 2003; Blankenship 2014; Croce and van Amerongen 2014). Both the overall composition and spatial organization of the photosynthetic apparatus play an important role in tuning the photosynthetic performance. A good understanding of the principles allowing photosynthetic organisms to live phototrophically at very different light conditions may help in our search for new ways to capture and utilize solar energy. The efficiency of solar cells, for instance, often declines with decreasing irradiance (Reich et al. 2011). In this respect, green sulfur bacteria are an excellent source of inspiration. Some of them are able to live at extremely low light intensities using photosynthesis as their sole source of energy (Manske et al. 2005): one species of green sulfur bacteria was found to live phototrophically using the dim radiation of black smokers at the bottom of the Pacific Ocean where no sunlight penetrates (Beatty et al. 2005). The main LH complex of green sulfur bacteria is a chlorosome, a large antenna with a unique structural organization of pigments. The core of the chlorosome contains tens to hundreds of thousands of bacteriochlorophyll (BChl) molecules arranged in self-assembling aggregates (Oostergetel et al. 2010; Orf and Blankenship 2013; Pšenčík et al. 2014). In all other known LH complexes pigment-binding proteins determine the positions and 


\section{Chapter 5}

orientations of only tens to hundreds of pigments. The aggregation of pigments in the chlorosome allows strong excitonic coupling between the pigments, accompanied by the formation of charge-transfer states (Frese et al. 1997) and is thought to be responsible for the light-harvesting and energy-transferring properties of the chlorosomes. However, the substantial disorder present in chlorosomes confines the strong coupling to relatively small domains. These domains are only weakly interacting (Dostál et al. 2012). Chlorobaculum (C.) tepidum is a model organism for this group of green sulfur bacteria and its chlorosome dimensions are typically 200x50x20 nm ${ }^{3}$, which makes chlorosomes the largest photosynthetic antennas. The size of the chlorosome together with the very high pigment concentration is apparently essential for efficient photon gathering at low-light conditions. However, efficient energy transfer of the formed excitations to the RCs is also crucial and that is why the overall arrangement of the photosynthetic apparatus is important (Oostergetel et al. 2010; Croce and van Amerongen 2014; Pšenčík and Mancal 2018). Green sulfur bacteria exhibit an unusual organization of their intermediate LH complexes. In contrast to higher plants, most of these LH complexes are peripheral, meaning that they are located outside the photosynthetic membrane (Blankenship and Matsuura 2003; Pšenčík and Mancal 2018). The excitation absorbed in a chlorosome interior is transferred to a number of additional antenna complexes of the chlorosome, together called the baseplate, which is formed by proteins binding $\mathrm{BChl}$ a and carotenoid molecules. The chlorosomes (including their baseplates) of green sulfur bacteria are attached to the cytoplasmic membrane via several so-called Fenna-Matthews-Olson (FMO) antenna complexes, through which the excitations are transferred to the RCs located in the cytoplasmic 
Efficiency of excitation-energy trapping in the reaction center of the low-light adapted green photosynthetic bacterium Chlorobaculum tepidum

membrane. The RC contains its own core antenna, which constitutes the only $\mathrm{LH}$ complex of green sulfur bacteria embedded in a membrane. The FMO complex and core antenna of the $\mathrm{RC}$ contain $\mathrm{BChl}$ a as the only chlorophyll-like pigment and together with the baseplate they exhibit highly overlapping spectra (Dostál et al. 2014).

The quantum efficiency of photochemistry, i.e. the excitation energy transfer to the $\mathrm{RC}$ and subsequent charge separation, in green sulfur bacteria is a matter of debate. Values close to $100 \%$ would be expected for low-light adapted organisms, as found for distantly related photosystem I or some photosynthetic bacteria (Wraight and Clayton 1974; Ruban et al. 2011; Kamen and Pietro 2013). On the other hand, the larger complexity of the photosynthetic apparatus of green sulfur bacteria, together with its extremely large size might lead to losses. Fluorescence excitation spectra revealed that the efficiency of energy transfer from $\mathrm{BChl} c$ to $\mathrm{BChl} a$ in whole cells of green sulfur bacteria is close to $100 \%$ at anaerobic conditions, which are natural to these bacteria (Wang et al. 1990a). However, at room temperature it is difficult to distinguish between the contributions of $\mathrm{BChl}$ a from the baseplate, the FMO complex and the core antenna, and therefore to decide whether the excitation was transferred with such a high efficiency from the chlorosome up to the RCs, or only to the baseplate or FMO complex. In measurements performed on isolated FMO-RC complexes or membranes the efficiency of energy transfer from the FMO complexes to the RCs has never been observed to be higher than $40 \%$ (He et al. 2015). Recently, the use of two-dimensional coherent spectroscopy allowed to distinguish several energy transfer steps in whole cells at $77 \mathrm{~K}$ and a much higher transfer efficiency of $75 \%$ was estimated (Dostál et al. 2016). This suggests that the 


\section{Chapter 5}

photosynthetic apparatus is vulnerable to damage during isolation and the use of whole cells is a prerequisite for obtaining realistic efficiencies. However, it was not possible to determine whether the less than $100 \%$ efficiency is a consequence of performing the experiments at low temperature, or whether it is an intrinsic property of the modular organization of the light-harvesting complexes. Here we report results of streak-camera measurements at $20^{\circ} \mathrm{C}$ and $45^{\circ} \mathrm{C}$ as well as at $77 \mathrm{~K}$ on whole cells of $C$. tepidum. The results indicate that at $20^{\circ} \mathrm{C}\left(45^{\circ} \mathrm{C}\right)$ the quantum efficiency of photochemistry is around $85-90 \%(80-86 \%)$, while the efficiency at $77 \mathrm{~K}$ is $(81-90 \%)$, which is somewhat higher than the results of Dostal et al (Dostál et al. 2016).

\section{Materials \& Methods}

\section{Sample Preparation}

C. tepidum cells, grown at $45^{\circ} \mathrm{C}$, were suspended in 10-20 mM Tris- $\mathrm{HCl}$ buffer, $\mathrm{pH}$ $=8.0$, and incubated with $5 \mathrm{mM}$ sodium dithionite inside an air-tight quartz cuvette for at least two hours before the time-resolved fluorescence measurements. Before and after each room-temperature $\left(20^{\circ} \mathrm{C}\right)$ and $45^{\circ} \mathrm{C}$ time-resolved fluorescence measurement, the absorption spectrum of the sample was recorded to check whether the sample had been exposed to oxygen, in which case the strong absorption of sodium dithionite at around $315 \mathrm{~nm}$ would be absent. Before performing the $77 \mathrm{~K}$ measurements, the absorption spectrum was also measured at room temperature.

\section{Absorption measurements}


Efficiency of excitation-energy trapping in the reaction center of the low-light adapted green photosynthetic bacterium Chlorobaculum tepidum

Absorption spectra of C. tepidum cells were recorded on a Cary 5E spectrophotometer, equipped with an integrating diffuse reflectance sphere (DRACA-50, Labsphere) to correct for light scattering by the cells. The optical path length was $1 \mathrm{~cm}$. All measurements were performed at room temperature.

\section{Time-resolved fluorescence measurements}

Picosecond fluorescence measurements were performed on $C$. tepidum cells at $20^{\circ} \mathrm{C}, 45^{\circ} \mathrm{C}$, and $77 \mathrm{~K}$ by using a synchroscan streak-camera system as described before (van Stokkum et al. 2008; Chukhutsina et al. 2015). At $20^{\circ} \mathrm{C}$, we used three time windows to record the fluorescence, namely 160 ps, 800 ps and 2000 ps while at $45^{\circ} \mathrm{C}$ and $77 \mathrm{~K}$ we used a time window of 2000 ps. At $20^{\circ} \mathrm{C}$, the instrument response function, IRF, was recorded before each measurement. The IRF was fitted with a sum of two or three Gaussian functions to describe the IRF mathematically as accurately as possible. The Full Width at Half Maximum (FWHM) of the Gaussian function with the largest contribution was 6 ps, 9 ps, 22 ps, at time windows 160 ps, $800 \mathrm{ps}$, and 2000 ps respectively. For the $45^{\circ} \mathrm{C}$ and $77 \mathrm{~K}$ measurements, the IRF was modelled as the sum of two Gaussian functions for which the largest FWHM was $\sim 22$ ps.

We used $400 \mathrm{~nm}$ excitation light to perform $20^{\circ} \mathrm{C}, 45^{\circ} \mathrm{C}$ and $77 \mathrm{~K}$ measurements. The detection efficiency of the streak camera is wavelength-dependent and all images were corrected for this dependency. The repetition rate of the laser was 4 $\mathrm{MHz}$. For $20^{\circ} \mathrm{C}\left(45^{\circ} \mathrm{C}\right)$ and $77 \mathrm{~K}$ measurements, the spot size was in the order of $\sim 40 \mu \mathrm{m}$ and $\sim 100 \mu \mathrm{m}$, respectively. At $20^{\circ} \mathrm{C}$ and $45^{\circ} \mathrm{C}$ (only performed with $2000 \mathrm{ps}$ 


\section{Chapter 5}

time window) the excitation energy per pulse for time windows 160 ps, 800 ps, and 2000 ps was $1.25 \mathrm{pJ}, 0.25-0.5 \mathrm{pJ}$, and $0.13-0.25 \mathrm{pJ}$, respectively. At $77 \mathrm{~K}$, the excitation energy per pulse was 0.5-1 pJ. All time-resolved fluorescence measurements lasted $20-40$ minutes. Several $20^{\circ} \mathrm{C}$ measurements with varying intensity were performed to assess at which excitation energy per pulse excitonexciton annihilation occurs (data not shown). At $2.5 \mathrm{pJ}$, the lifetimes were slightly shorter indicating a small amount of exciton-exciton annihilation at this intensity.

For $20^{\circ} \mathrm{C}\left(45^{\circ} \mathrm{C}\right)$ and $77 \mathrm{~K}$ measurements, the optical density of the samples was $0.15-0.2$ and $\sim 0.8$ at $740 \mathrm{~nm}$, respectively, measured at room temperature in a quartz cuvette with $1 \mathrm{~cm}$ optical path length. The $20^{\circ} \mathrm{C}\left(45^{\circ} \mathrm{C}\right)$ time-resolved measurements were done using a quartz cuvette with an optical path of $1 \mathrm{~cm}$. For $77 \mathrm{~K}$ time-resolved measurements, the samples were collected quickly from a quartz cuvette (only used to keep the cells in anaerobic environment for $77 \mathrm{~K}$ measurements) in a glass Pasteur pipette with $\sim 1 \mathrm{~mm}$ diameter and frozen using liquid nitrogen. Measurements were performed on the sample in the Pasteur pipette. The excitation light was vertically polarized and the polarizer in the detection branch was set to magic angle. For $20^{\circ} \mathrm{C}$ and $45^{\circ} \mathrm{C}$ time-resolved measurements a Nikon CFI Plan Apo Lambda10X objective lens with a numerical aperture of 0.45 and focal length of $\sim 20 \mathrm{~mm}$ was used. For $77 \mathrm{~K}$ time-resolved measurements a lens with a focal length of $7 \mathrm{~cm}$ was used. 
Efficiency of excitation-energy trapping in the reaction center of the low-light adapted green photosynthetic bacterium Chlorobaculum tepidum

\section{Target and global analysis}

Global and target analyses were performed as described in (Tian et al. 2011, 2012), using an in-house program written in Matlab. As part of this program, OPTI TOOLBOX (Currie and Wilson 2012) was used.

In order to resolve the different stages of the excitation energy transfer (EET) from the chlorosome to the baseplate and FMO complex at room temperature, we performed simultaneous global analysis on the time-resolved data obtained with 160 ps and 800 ps time windows.

The $20^{\circ} \mathrm{C}$ measurements obtained with $2000 \mathrm{ps}$ time window were analyzed together globally with three lifetimes. Then, the slowest rate obtained from this global analysis was fixed in the global analysis of $20^{\circ} \mathrm{C}$ measurements with $160 \mathrm{ps}$ and $800 \mathrm{ps}$ time window that were fitted together with four rates.

For the analysis of the $20^{\circ} \mathrm{C}$ data four lifetime were used and it was imposed that the DAS should have the same shape in all different measurements.

For the analysis of the $77 \mathrm{~K}$ data, all measured with a $2000 \mathrm{ps}$ time window, it was also imposed that the decay-associated spectra (DAS) obtained with different measurements should have the same shape. Three lifetimes were enough to fit the data. 


\section{Chapter 5}

\section{Results \& Discussion}

\section{Time-resolved fluorescence of C. tepidum cells at $20^{\circ} \mathrm{C}$ and $77 \mathrm{~K}$}

Time-resolved fluorescence spectroscopy enables to monitor EET between BChl $c$ aggregates inside the chlorosomes and $\mathrm{BChl} a$ in the baseplate, FMO complexes and the core antenna of the RC. BChls $c$ constitute $\sim 97 \%$ of the total BChl content in C. tepidum, the other $\sim 3 \%$ is $\mathrm{BChl}$ a. Out of this $3 \%, 1 \%$ is located in the baseplate and the remaining $2 \%$ is present in the $\mathrm{FMO}$ complexes and $\mathrm{RC}$ cores (Frigaard et al. 2003; Pšenčík and Mancal 2017).

Global analysis of $20^{\circ} \mathrm{C}$ and $77 \mathrm{~K}$ time-resolved fluorescence data provides a modelindependent insight into EET in intact cells. Figures 1a-b and 1c show the results of global analysis of the $20^{\circ} \mathrm{C}$ and $77 \mathrm{~K}$ time-resolved fluorescence data. All the DAS in Figure 1a and 1c are normalized to the maximum of their time-zero spectrum. The time-zero spectrum is the summation of all DAS obtained from the analysis of one sample. This spectrum is close to the fluorescence spectrum directly after excitation and relaxation to the $Q_{y}$ states, provided that no additional relaxation processes occur.

All the DAS in Figure 1b, taken from Figure 1a, are normalized to their maxima for a better comparison of the spectral shape.

Since BChl $c$ represents the vast majority of all chlorophylls in the chlorosomes, the $400 \mathrm{~nm}$ laser pulses mainly excite BChl $c$ and to a lesser extent BChl a. Initially, the emission is therefore almost exclusively due to $\mathrm{BChl} c$. Then, the excitation energy migrates from the chlorosome interior to the baseplate and via the FMO complex to 
Efficiency of excitation-energy trapping in the reaction center of the low-light adapted green photosynthetic bacterium Chlorobaculum tepidum

the core antenna of the RCs, which leads to a rise of the fluorescence at $800-840$ $\mathrm{nm}$ after tens of picoseconds. In the experiments we performed, the fluorescence of $\mathrm{BChl} a$ in the baseplate could not be separated from the fluorescence of $\mathrm{BChl} a$ in the FMO complex and the cores of RCs. This is in agreement with the observation that the distributions of energy levels in all these three LH complexes are highly overlapping (Dostál et al. 2014), despite the fact that the fluorescence maxima for baseplate, FMO complex, and RC core differ: $\sim 810, \sim 825$ and $\sim 835 \mathrm{~nm}$ respectively (Francke et al. 1996; Pšenčík et al. 2003; Hohmann-Marriott and Blankenship 2007; Orf et al. 2014).

As a consequence, from the DAS obtained by global analysis, only energy transfer from $\mathrm{BChl} c$ to $\mathrm{BChl}$ a can be resolved, which manifests itself as a positive peak at shorter wavelengths and a negative peak at longer wavelengths where $\mathrm{BChl} c$ and $\mathrm{BChl}$ a emit their fluorescence, respectively. The presence of positive and negative peaks in the same DAS, is seen in two of the $20^{\circ} \mathrm{C}$ DAS in Figure $1 \mathrm{a}$ and one of the $77 \mathrm{~K} \mathrm{DAS}$ in Figure 1c. Both $20^{\circ} \mathrm{C}$ DAS indicate EET from BChl $c$ in the chlorosome, with a positive peak at $768-773 \mathrm{~nm}$, to the $\mathrm{BChl}$ a containing complexes, with negative peaks at $800-820$ for the minor and $805-838 \mathrm{~nm}$ for the major component, and rates of $(13 \mathrm{ps})^{-1}$ and $(49 \mathrm{ps})^{-1}$, respectively. Rates of $\sim(30-40 \mathrm{ps})^{-1}$ for EET from $\mathrm{BChl} c$ to $\mathrm{BChl} a$, similar to the dominant $(49 \mathrm{ps})^{-1}$ component in the present work, were reported in other experiments performed on isolated chlorosomes (Causgrove et al. 1992; van Noort et al. 1997). In Figure 1c, the $77 \mathrm{~K}$ DAS reflecting EET has a rate of (42 ps) $)^{-1}$ with a positive peak at $\sim 783 \mathrm{~nm}$ and a negative one at $\sim 818-838 \mathrm{~nm}$. This is again indicative of EET from BChl $c$ to $\mathrm{BChl}$ a. Shorter lifetimes were not 
detected, because only a 2000 ps window was used at $77 \mathrm{k}$ leading to the of the broad width ( 24 ps FWHM) of the IRF. Another recent study (Dostál et al. 2016) on intact cells reported a somewhat slower rate of $(70 \mathrm{ps})^{-1}$ at $77 \mathrm{~K}$ for EET from BChl $c$ to $\mathrm{BChl} a$ in the FMO complex.
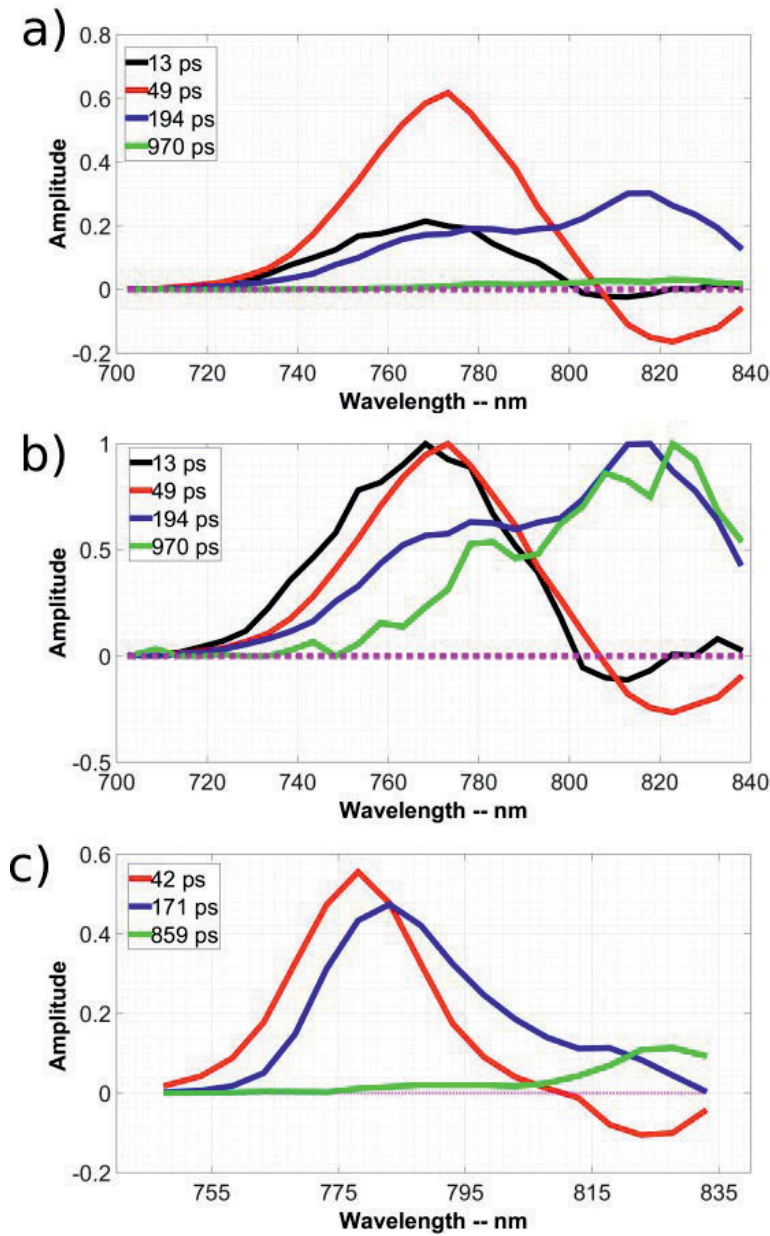

Figure 1. a) Decay-associated spectra (DAS) obtained by global analysis of room temperature time-resolved fluorescence measurements on C. tepidum cells. b) Each DAS taken from a is normalized to its maximum. c) DAS obtained by global analysis of $77 \mathrm{~K}$ time-resolved fluorescence measurements on C. tepidum cells. The excitation wavelength was $400 \mathrm{~nm}$ both at $20^{\circ} \mathrm{C}$ and $77 \mathrm{~K}$. All DAS in a) and c) are normalized to the maximum of the corresponding time-zero spectrum, i.e. 
Efficiency of excitation-energy trapping in the reaction center of the low-light adapted green photosynthetic bacterium Chlorobaculum tepidum

the sum of all DAS was normalized to 1 in the maximum. The inset shows the lifetimes in ps corresponding to the DAS represented with the same color. The dotted purple line is the zero line.

So far we looked at EET from BChl $c$ to BChl $a$ in the baseplate, FMO complex and the core antenna of the RC. The excitation energy finally reaches the RCs, where it leads to trapping of the excitation energy and no fluorescence emission is possible anymore. The $20^{\circ} \mathrm{C}(194 \mathrm{ps})^{-1}$ DAS reflects the disappearance of excitation energy, that is "equilibrated" over all spectral species, due to trapping in the RC. It has a positive peak at $\sim 773 \mathrm{~nm}$ due to $\mathrm{BChl} c$ and another positive peak at $\sim 814 \mathrm{~nm}$ due to $\mathrm{BChl}$ a. At $77 \mathrm{~K}$, trapping in the RCs is reflected by the $(171 \mathrm{ps})^{-1} \mathrm{DAS}$ with one positive peak at $788 \mathrm{~nm}$ and another positive peak at $823 \mathrm{~nm}$. The peak at $823 \mathrm{~nm}$ reflects the excitation energy of $\mathrm{BChl}$ a that is being trapped in the $\mathrm{RCs}$. Finally, there is a very small $20^{\circ} \mathrm{C}$ DAS with a decay rate of $(970 \mathrm{ps})^{-1}$. The $(970 \mathrm{ps})^{-1}$ DAS has two positive peaks at $778-783 \mathrm{~nm}$ and at $800-840 \mathrm{~nm}$. This DAS has a similar shape as the $20^{\circ} \mathrm{C}(194 \mathrm{ps})^{-1}$ component (see Figure $\left.1 \mathrm{~b}\right)$ and represents slow trapping in the RCs. Less amplitude at $778-783 \mathrm{~nm}$ indicates slower back transfer of excitation energy to $\mathrm{BChl} c$ in comparison to the $20^{\circ} \mathrm{C}(194 \mathrm{ps})^{-1} \mathrm{DAS}$. The $77 \mathrm{~K}(859 \mathrm{ps})^{-1}$ DAS has a maximum at longer wavelengths in comparison to the $77 \mathrm{~K}(171 \mathrm{ps})^{-1} \mathrm{DAS}$ and it reflects BChls a that trap the excitation energy slower (relative to (171 ps)-1 DAS) at this low temperature. The corresponding DAS at room temperature where uphill energy transfer to the reaction center occurs more easily, has a very small amplitude (see Figure 1a).

We calculated the $20^{\circ} \mathrm{C}$ absorption spectrum of cells from the time-zero fluorescence spectrum obtained from global analysis of $20^{\circ} \mathrm{C}$ measurements (See 
Figure S1 for the time-zero spectrum at $77 \mathrm{~K}$ ) applying the Kennard-Stepanov relation (Larkum, Douglas, and Raven 2003), (see Figure 2). The absorption spectrum that is calculated in this way almost perfectly overlaps with the measured $20^{\circ} \mathrm{C}$ absorption spectrum of the $\mathrm{BChl} c \mathrm{Q}_{\mathrm{y}}$ absorption of the chlorosomes, meaning that the excitations at "time zero" are already spectrally equilibrated over the chlorosome with a rate faster than the $\sim 6 \mathrm{ps}$ time-resolution, of the streak-camera setup. The absence of Bchl a absorption in the calculated absorption spectra reflects the fact that EET to Bchl a occurs on slower time scale (see the 13 ps and 49 ps DAS in Figure 1a).

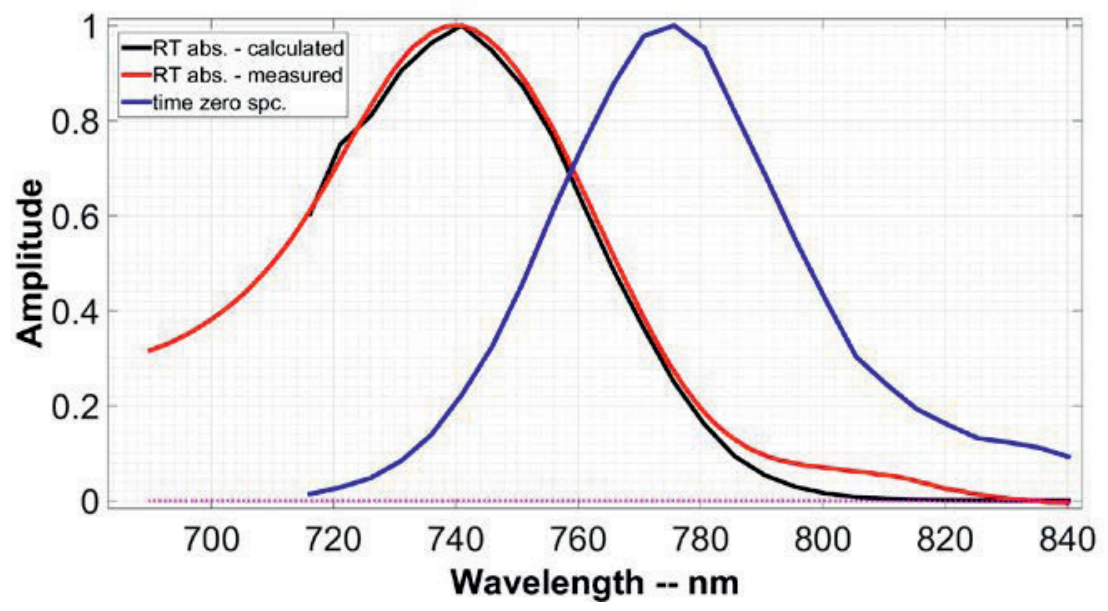

Figure 2: The calculated (see text) absorption spectrum in black is compared to the measured absorption spectrum in red. The blue curve shows RT time-zero spectrum calculated from the RT $\left(20^{\circ} \mathrm{C}\right)$ global analysis. The dotted purple line is the baseline.

\section{Target analysis of $77 \mathrm{~K}$ time-resolved data of $C$. tepidum cells}

The time-resolved fluorescence at $77 \mathrm{~K}$ shows a shift in the $\mathrm{BChl} c$ peak from $\sim 784$ $\mathrm{nm}$ to $\sim 788 \mathrm{~nm}$ over a time period of $\sim 50 \mathrm{ps}$ (see Figure S2). Thus, we need at least 
Efficiency of excitation-energy trapping in the reaction center of the low-light adapted green photosynthetic bacterium Chlorobaculum tepidum

two compartments to represent $\mathrm{BChl} c$. We assumed the shape of the two corresponding spectra representing $\mathrm{BChl} c$ to be the same with a separation of $5 \mathrm{~nm}$ relative to each other and they were forced to be zero between $830 \mathrm{~nm}$ to $840 \mathrm{~nm}$, where $\mathrm{BChl} c$ hardly contributes to the emission. At least two spectral forms of $\mathrm{BChl}$ a exist, with decay times of 171 ps and 856 ps, respectively, and which are spectrally shifted by about $10 \mathrm{~nm}$ (see Figure 1c). Thus, two compartments of BChl a were also included in the target analysis. Similar as for BChl $c$, the spectra of both compartments were required to have the same shape, but they were separated by $10 \mathrm{~nm}$ from each other. The spectra of BChl a were forced to zero between $750 \mathrm{~nm}$ and $790 \mathrm{~nm}$.

We performed a target analysis simultaneously on four streak images recorded for four different samples with a 2000 ps time window. Figure 3 shows the model used for the target analysis and the resulting rates and species associated spectra (SAS). We chose the initial population according to the relative content of BChl $c$ and $\mathrm{BChl}$ $a$ in the cells. The initial population of $\mathrm{BChl} c$ is 0.97 . The best fit is obtained if this initial population is evenly distributed over the two compartments that represent $\mathrm{BChl}$ c. The initial population of $\mathrm{BChl} a$ is 0.03 . There are two $\mathrm{BChl}$ a compartments; one has a lower energy than the other Bchl a compartment. In (Dostál et al. 2016), the authors concluded that $25 \%$ of the excitations are trapped on the FMO complexes. We assumed that excitation is equilibrated over all three $\mathrm{BChl}$ a-containing complexes with highly overlapping spectra. According to the global analysis, EET from BChl $a$ to the RCs occurs with two time constants, being 171 ps and 859 ps. For the $77 \mathrm{~K}$ measurements we assumed that one third of the BChls a (i.e. $33 \%$ of 


\section{Chapter 5}

0.03) transfers to the RCs with a slow time constant whereas the other fraction of 0.02 corresponds to BChls a that transfer their excitation energy to the RCs faster.

In Figure 3 it is shown that the energy is transferred from the black $(\mathrm{BChl} c)$ to the blue $\left(\mathrm{BChl}\right.$ a) compartment with a rate of $(42 \mathrm{ps})^{-1}$. The obtained back transfer rate for the black compartment was extremely small and we set it to zero. The red (BChl

c) compartment transfers its excitations to the blue compartment with a rate of (148 ps $)^{-1}$ and the reverse process occurs with a rate of $(877 \mathrm{ps})^{-1}$.

The blue compartment decays due to charge separation in the RC with a rate of (188 ps) $)^{-1}$. Moreover, the blue compartment transfers its excitation energy to the green one with a rate of $(440 \mathrm{ps})^{-1}$. The latter in turn decays with a rate of $(1520 \mathrm{ps})^{-1}$.

Each compartment in Figure 3 dissipates the excitation energy with a rate of (2.5 ns)${ }^{1}$ (not shown). Assuming that both the blue and green compartments participate in trapping, the trapping efficiency is $80 \%$ (see Figure S3 for the kinetics of each compartment). This efficiency is calculated by integrating the population of the blue and green compartments over time until there is no excitation energy in any compartments. Dostál et al. 2016 estimated that $25 \%$ of the excitation energy remain trapped in the lowest energy of the FMO complexes and hence the EET efficiency from the FMO complexes to the RC is $75 \%$. The authors stated that the excitonexciton annihilation could have affected their estimate of this efficiency as well as trapping of the excitation energy on FMOs due to the low temperature. The excitation energy per pulse in the present study is 1000 times smaller than what was used in (Dostál et al. 2016), which eliminates nearly all exciton-exciton annihilation. In order 
Efficiency of excitation-energy trapping in the reaction center of the low-light adapted green photosynthetic bacterium Chlorobaculum tepidum

to assess if trapping at low temperature plays a role, we performed target analysis on time-resolved measurements performed on the cells at $20^{\circ} \mathrm{C}$ (see next section).

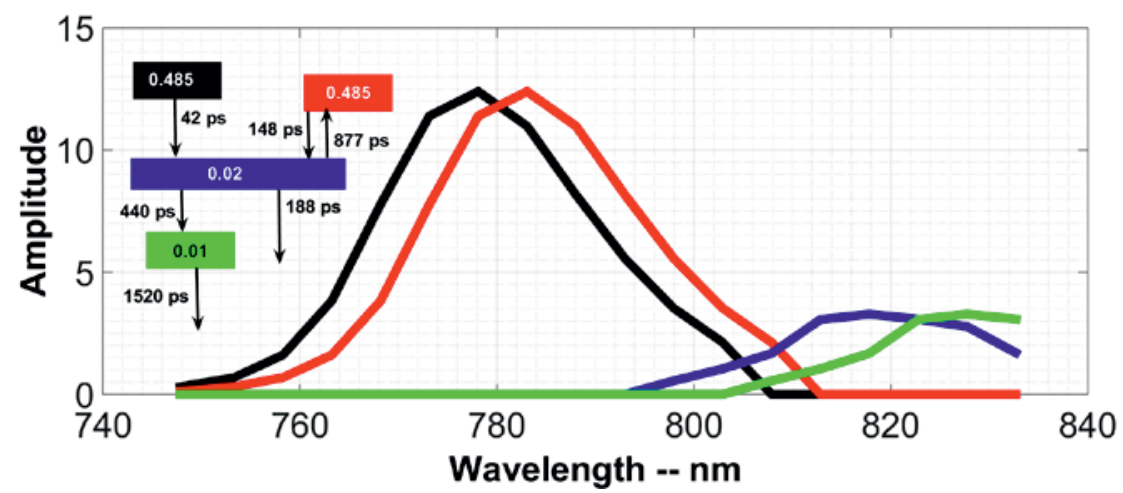

Figure 3. $77 \mathrm{~K}$ target model and corresponding SAS of C. tepidum. The colors of the boxes representing the compartments are the same as for the corresponding SAS. The black and red spectra correspond to $\mathrm{BChl} c$. The blue and green spectra correspond to $\mathrm{BChl}$ a. The initial population of each compartment is written inside the blocks representing the compartments.

\section{Target analysis of $20^{\circ} \mathrm{C}$ time-resolved data of $\mathrm{C}$. tepidum cells}

The positive and negative peaks of the $(13 \mathrm{ps})^{-1}$ and $(49 \mathrm{ps})^{-1}$ DAS showed two spectrally-different EET events from BChl $c$ in the chlorosome to $\mathrm{BChl} a$ in the baseplate and/or FMO and we considered two compartments for BChl $c$ and two for $\mathrm{BChl}$ a. Figure 4 shows the target model used for $20^{\circ} \mathrm{C}$ time-resolved measurements together with the corresponding rates and SAS.

One possible interpretation of the two compartments for $\mathrm{BChl} c$ is that one comprises the domains of aggregates located in the vicinity of the baseplate (transfer time 11 ps) and the other comprises the domains located in the rest of the chlorosome (transfer time $54 \mathrm{ps).} \mathrm{While} \mathrm{for} \mathrm{the} \mathrm{former} \mathrm{domains} \mathrm{it} \mathrm{is} \mathrm{possible} \mathrm{to} \mathrm{transfer} \mathrm{excitation}$ 


\section{Chapter 5}

energy almost directly to $\mathrm{BChl} a$ in the baseplate, the excitations in the latter have to undergo a random walk throughout the large chlorosome before they get close to the baseplate. As the formation of the aggregates is based on self-assembly, there is no obvious way how to establish an energy gradient within the chlorosome. Since the majority of BChl $c$ molecules are not in contact with the baseplate, this interpretation also explains why the slower component possesses a larger amplitude. Similar results were obtained for chlorosomes of $\mathrm{BChl}$ e containing Chlorobium phaeobacteroides (Pšenčík et al. 2003). The two exponential decays may also be (partly) caused by the static disorder and dynamic fluctuations among the many thousands of pigment molecules in the large chlorosome, which inevitably leads to a multi-exponential decay (Chmeliov et al. 2014).

The (970 ps) $)^{-1}$ DAS seems to be due to BChls a that temporarily "trap" the excitation energy and transfer their excitation to RCs with a slow time constant, which is far more pronounced at $77 \mathrm{~K}$ as explained before. One of the $\mathrm{BChl}$ a compartments represents this group of $\mathrm{BChl}$ a. The other $\mathrm{BChl}$ a compartment represents the $\mathrm{BChls}$ a that transfer excitation energy to the RC, where trapping occurs, on a faster timescale. In the fitting, we connected the BChls a participating in slow trapping compartment only to the compartment of BChls a that participate in trapping with a faster time constant, which is somewhat arbitrary but not limiting for the conclusions drawn below. The spectral shape of the SAS representing the two BChl a compartments was taken to be the same with a $5 \mathrm{~nm}$ shift relative to each other to approximate the energy difference between the two pools. Furthermore, the BChl a spectrum was set to zero between $700 \mathrm{~nm}$ and $750 \mathrm{~nm}$, in accordance with its emission spectrum. 
Efficiency of excitation-energy trapping in the reaction center of the low-light adapted green photosynthetic bacterium Chlorobaculum tepidum

We chose the initial population of these compartments based on the percentages of $\mathrm{BChl} c$ and $a$ in the cells. The initial population of the green compartment, representing BChl a with slow trapping time, was set to 0.01 . This choice does not affect the results and it could also be zero.

In Figure 4, the black and red SAS belong to the black and red compartments, respectively, corresponding to $\mathrm{BChl} c$ in the chlorosome. The blue and green SAS belong to the blue and green compartments, respectively, corresponding to $\mathrm{BChl}$ a of the baseplate, FMO complexes, and core antenna of the RC.

The energy transfer from the black $\mathrm{BChl} c$ compartment to the blue $\mathrm{BChl} a$ compartment is characterized by a rate of $(11 \mathrm{ps})^{-1}$. The back transfer process has a rate of $(460 \mathrm{ps})^{-1}$ (see SI for more details). The forward and backward EET rate from the red $\mathrm{BChl} c$ compartment to and from the blue $\mathrm{BChl}$ a compartment are (54 ps $)^{-1}$ and $(600 \mathrm{ps})^{-1}$, respectively.

The blue $\mathrm{BChl}$ a compartment decays largely due to charge separation in the $\mathrm{RC}$ with a rate of $(220 \mathrm{ps})^{-1}$. In addition, the blue $\mathrm{BChl}$ a compartment transfers its excitation energy to the green $\mathrm{BChl}$ a compartment with a rate of $(2950 \mathrm{ps})^{-1}$. The decay rate of the green $\mathrm{BChl}$ a compartment, representing also BChls a that lose their excitation energy due to trapping but on a slower time scale, was fixed in the target analysis to $(970 \mathrm{ps})^{-1}$ that was obtained from global analysis. The blue $\mathrm{BChl}$ a compartment represents $\mathrm{BChl} a$ in the baseplate, FMO complexes, and the core antenna of the RC. The excitation energy is equilibrated over the BChls a in all complexes due to their large spectral overlap (Dostál et al. 2014). For the same 


\section{Chapter 5}

reason also the trap states from the green $\mathrm{BChl}$ a compartment may be found in all three complexes. Energy transfer from these traps towards the RC probably requires thermal energy and consequently, the contribution of the low-energy trap states is more significant at $77 \mathrm{~K}$ (see previous section). Each compartment in Figure 3 dissipates the excitation energy with a rate of $(2.5 \mathrm{~ns})^{-1}$ (He et al. 2015). Assuming that both blue and green compartments participate in trapping, the efficiency of trapping is $88 \%$ (see Figure S4 for the kinetics of each compartment). The efficiency was calculated by integrating the population of the compartments representing Bchl a over time until there was no excitation energy in the compartments. The trapping efficiencies at $20^{\circ} \mathrm{C}$ and $77 \mathrm{~K}$ do not considerably differ. To have a modelindependent way of calculating the trapping efficiency, in the next section we used global analysis of $20^{\circ} \mathrm{C}$ and $77 \mathrm{~K}$ time-resoled measurements (see Figure 1).

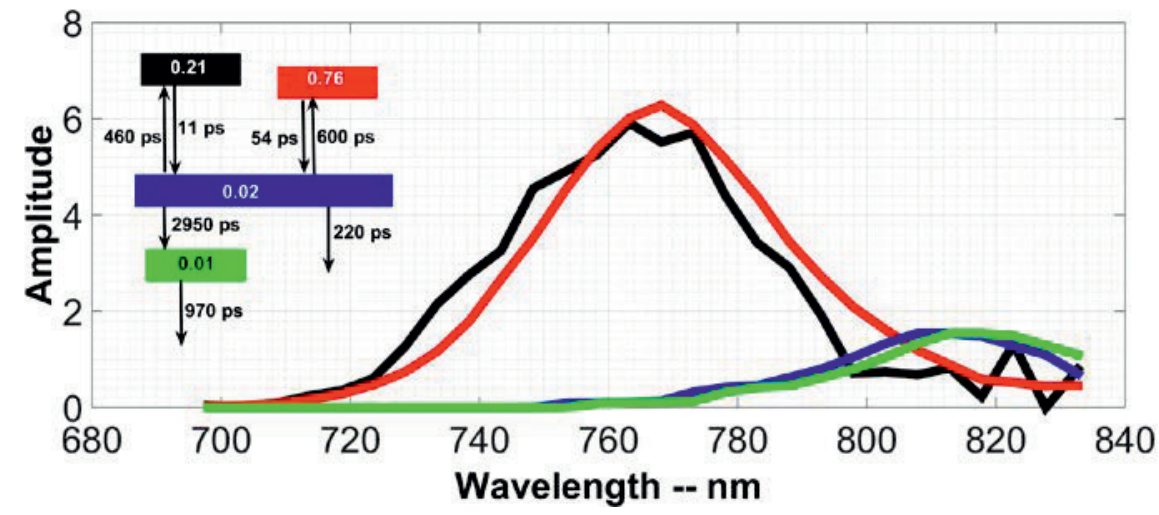

Figure 4: a) Room temperature target model and the obtained corresponding SAS for $C$. tepidum. Each spectrum is shown with the same color as the corresponding compartment. The black and red compartments represent $\mathrm{BChl} c$. The green and blue compartments correspond to $\mathrm{BChl} a$. The initial fractional populations are written in the four compartments. For each compartment the excitation energy dissipates with a rate of $(2.5 \mathrm{~ns})^{-1}$, which is not shown. 
Efficiency of excitation-energy trapping in the reaction center of the low-light adapted green photosynthetic bacterium Chlorobaculum tepidum

\section{Charge separation and trapping efficiency at $20^{\circ} \mathrm{C}, 45^{\circ} \mathrm{C}$, and $77 \mathrm{~K}$}

The quantum efficiency of photochemistry (i.e. the number of absorbed photons being transferred to the RC and leading to charge separation) in the studied cells of

C. tepidum can be estimated from $\Phi_{\mathrm{CS}}=\frac{\text { DAS } S_{\text {trapping } 1}}{\text { DAS }_{\text {trapping } 1}+\text { DAS } S_{\text {trapping }} 2}\left(1-\frac{\tau_{\text {trapping } 1}}{\tau_{\text {int }}}\right)+$ $\frac{D A S_{\text {trapping } 2}}{D A S_{\text {trapping } 1}+D A S_{\text {trapping } 2}}\left(1-\frac{\tau_{\text {trapping } 2}}{\tau_{\text {int }}}\right) e q 1$. where $\tau_{\text {trapping } 1}\left(\tau_{\text {trapping } 2}\right)$ and $\tau_{\text {int }}$ are the lifetime of the trapping DAS and the intrinsic lifetime of $\mathrm{BChl}$ a. Intrinsic lifetime is considered to be the decay time of the excited state of a species due to only fluorescence without any contribution from EET, internal conversion or other processes that shorten the lifetime of excited state. DAS $S_{\text {trapping } 1}$ and DAS trapping 2 are the areas under the corresponding DAS that represent trapping. For example, at $20^{\circ} \mathrm{C}$ we consider the (194 ps) $)^{-1}$ and $(970 \mathrm{ps})^{-1}$ DAS (see Figure 1a) to be due to trapping and the DAS representing trapping at $77 \mathrm{~K}$ are the $(171 \mathrm{ps})^{-1}$ and $(859 \mathrm{ps})^{-}$ ${ }^{1}$ DAS. We make use of an earlier finding that the efficiency of BChl c-to-BChl a EET is $\sim 100 \%$. (Wang et al. 1990b). We consider only the situation that excitations arrive to the reduced RC, in other words, the light intensity should be low enough to ensure that the excitation flow in the photosynthetic apparatus is slower than the turnover of the RC. At higher light intensities, the excitation may arrive to the RC in its oxidized state. In such a case the excitation will be quenched with the same rate constant, but the process will not lead to charge separation. This assumption is based on the analogy with a similar photosystem I (Schlodder et al. 2011). We take the intrinsic lifetime of fluorescence as $2.5 \mathrm{~ns}$ (He et al. 2015). 


\section{Chapter 5}

Table 1 shows a summary of the trapping efficiency calculated in this study. The trapping efficiency at $77 \mathrm{~K}$ and $20^{\circ} \mathrm{C}$ is, respectively, $85-90 \%$ and $81-90 \%$. If we take the intrinsic fluorescence lifetime to be 2000 ps, then the trapping efficiencies at 77 $\mathrm{K}$ is calculated to be $76 \%$ which is more in agreement with the efficiency calculated in (Dostál et al. 2016) . These results strongly suggest that the trapping efficiencies at $77 \mathrm{~K}$ and $20^{\circ} \mathrm{C}$ do not differ considerably. However, as is obvious from the global analysis, the overall trapping time gets significantly shorter at $20^{\circ} \mathrm{C}$. This is a consequence of the fact that thermal energy is required for the energy transfer from the low-energy BChl a trap states to RCs. The lower-energy BChl a compartment, which was used in the target analysis to describe these traps, is shifted by $\sim 75 \mathrm{~cm}^{-1}$ from the higher-energy one, and $\mathrm{kT}$ corresponds to $\sim 55 \mathrm{~cm}^{-1}$ at $77 \mathrm{~K}$ whilst it is $\sim 205$ $\mathrm{cm}^{-1}$ at $20^{\circ} \mathrm{C}$.

Table 1: A summary of the calculated trapping efficiencies at different temperatures.

\begin{tabular}{|l|c|}
\hline \multicolumn{1}{|c|}{ Temperatures } & Efficiencies \\
& \\
\hline $77 \mathrm{~K}$ & $81-90 \%$ \\
\hline $20^{\circ} \mathrm{C}$ & $85-90 \%$ \\
\hline $45^{\circ} \mathrm{C}$ & $80-86 \%$ \\
\hline
\end{tabular}

\section{Light harvesting in green bacteria at low-light conditions}

In a previous study on EET in whole cells of $C$. tepidum, it was shown that the bottleneck of EET is the transfer between the complexes forming the $\mathrm{LH}$ apparatus 
Efficiency of excitation-energy trapping in the reaction center of the low-light adapted green photosynthetic bacterium Chlorobaculum tepidum

(Dostál et al. 2016). Therefore it might seem surprising that this low-light adapted bacterium employs three $\mathrm{LH}$ complexes containing $\mathrm{BChl}$ a (baseplate, FMO complexes and the core antenna), especially if their spectra are highly overlapping. Such an organization does not have any advantage from an energetic point of view, but must be necessary for another reason. The main function of the FMO complexes is probably to serve as a spacer between the chlorosome and the cytoplasmic membrane to allow access of ferredoxin to the RC and at the same time to ensure excitation flow between the chlorosome baseplate and the RC in the cytoplasmic membrane (Wen et al. 2009; Dostál et al. 2014). It has been argued before that the baseplate in a chlorosome substantially improves the photosynthetic efficiency by funneling excitation to the reaction centers (Oostergetel et al. 2010). Our results suggest that green sulfur bacteria have developed a way of minimizing the losses related to EET between the three complexes. The DAS and the target model reported in this work suggest that all $\mathrm{BChl}$ a molecules (except the low-energy ones) form one functional compartment implying that the overlapping distributions of energy levels in the baseplate, the FMO complexes and the core antenna lead to the formation of a functional "super-complex" of BChl-a containing complexes, and to fast equilibration of the excitations over the whole $\mathrm{BChl}$ a supercomplex. This may prevent the slowdown of EET at the boundaries between the complexes, leading to high efficiency of trapping in the RCs over a wide range of temperatures.

\section{References:}

Beatty JT, Overmann J, Lince MT, et al (2005) An obligately photosynthetic bacterial anaerobe from a deep-sea hydrothermal vent. Proc Natl Acad Sci United States Am 102:9306-9310. doi: 10.1073/pnas.0503674102 


\section{Chapter 5}

Blankenship RE (2014) Molecular Mechanisms of Photosynthesis. Wiley

Blankenship RE, Matsuura K (2003) Antenna Complexes from Green Photosynthetic Bacteria. In: Green BR, Parson WW (eds) Light-Harvesting Antennas in Photosynthesis. Springer Netherlands, Dordrecht, pp 195-217

Causgrove TP, Brune DC, Blankenship RE (1992) Förster energy transfer in chlorosomes of green photosynthetic bacteria. J Photochem Photobiol B Biol 15:171-179. doi: https://doi.org/10.1016/1011-1344(92)87014-Z

Chmeliov J, Trinkunas G, van Amerongen H, Valkunas L (2014) Light Harvesting in a Fluctuating Antenna. J Am Chem Soc 136:8963-8972. doi: 10.1021/ja5027858

Chukhutsina V, Bersanini L, Aro E-M, van Amerongen H (2015) Cyanobacterial Light-Harvesting Phycobilisomes Uncouple From Photosystem I During DarkTo-Light Transitions. Sci Rep 5:14193. doi: 10.1038/srep14193

Croce R, van Amerongen $\mathrm{H}$ (2014) Natural strategies for photosynthetic light harvesting. Nat Chem Biol 10:492-501

Currie J, Wilson DI (2012) OPTI: lowering the barrier between open source optimizers and the industrial MATLAB user. Found Comput Process Oper $24: 32$

Dostál J, Mančal T, Augulis R, et al (2012) Two-Dimensional Electronic Spectroscopy Reveals Ultrafast Energy Diffusion in Chlorosomes. J Am Chem Soc 134:11611-11617. doi: 10.1021/ja3025627

Dostál J, Pšenčík J, Zigmantas D (2016) In situ mapping of the energy flow through the entire photosynthetic apparatus. Nat Chem 8:705-710. doi: 10.1038/nchem.2525

Dostál J, Vácha F, Pšenčík J, Zigmantas D (2014) 2D Electronic Spectroscopy Reveals Excitonic Structure in the Baseplate of a Chlorosome. J Phys Chem Lett 5:1743-1747. doi: 10.1021/jz5005279

Francke C, Otte SCM, Miller M, et al (1996) Energy transfer from carotenoid and FMO-protein in subcellular preparations from green sulfur bacteria. Spectroscopic characterization of an FMO-reaction center core complex at low temperature. Photosynth Res 50:71-77. doi: 10.1007/BF00018222

Frese R, Oberheide U, van Stokkum I, et al (1997) The organization of bacteriochlorophyll $\mathrm{c}$ in chlorosomes from Chloroflexus aurantiacus and the structural role of carotenoids and protein. Photosynth Res 54:115-126. doi: 10.1023/A:1005903613179

Frigaard N-U, Chew AGM, Li H, et al (2003) Chlorobium Tepidum: Insights into the Structure, Physiology, and Metabolism of a Green Sulfur Bacterium Derived 
Efficiency of excitation-energy trapping in the reaction center of the low-light adapted green photosynthetic bacterium Chlorobaculum tepidum

from the Complete Genome Sequence. Photosynth Res 78:93-117. doi: 10.1023/B:PRES.0000004310.96189.b4

He G, Niedzwiedzki DM, Orf GS, et al (2015) Dynamics of Energy and Electron Transfer in the FMO-Reaction Center Core Complex from the Phototrophic Green Sulfur Bacterium Chlorobaculum tepidum. J Phys Chem B 119:83218329. doi: 10.1021/acs.jpcb.5b04170

Hohmann-Marriott MF, Blankenship RE (2007) Variable fluorescence in green sulfur bacteria. Biochim Biophys Acta - Bioenerg 1767:106-113. doi: https://doi.org/10.1016/j.bbabio.2006.11.011

Huner NPA, Öquist G, Melis A (2003) Photostasis in Plants, Green Algae and Cyanobacteria: The Role of Light Harvesting Antenna Complexes. In: Green BR, Parson WW (eds) Light-Harvesting Antennas in Photosynthesis. Springer Netherlands, Dordrecht, pp 401-421

Kamen MD, Pietro AS (2013) Primary Processes in Photosynthesis. Elsevier Science

Larkum, A., Douglas, S. and Raven JA eds. (2003) Photosynthesis in algae (Vol. 14). Springer Science \& Business Media.

Manske AK, Glaeser J, Kuypers MMM, Overmann J (2005) Physiology and Phylogeny of Green Sulfur Bacteria Forming a Monospecific Phototrophic Assemblage at a Depth of 100 Meters in the Black Sea. Appl Environ Microbiol 71:8049-8060. doi: 10.1128/AEM.71.12.8049-8060.2005

Oostergetel GT, van Amerongen H, Boekema EJ (2010) The chlorosome: a prototype for efficient light harvesting in photosynthesis. Photosynth Res 104:245-255. doi: 10.1007/s11120-010-9533-0

Orf GS, Blankenship RE (2013) Chlorosome antenna complexes from green photosynthetic bacteria. Photosynth Res 116:315-331. doi: 10.1007/s11120013-9869-3

Orf GS, Niedzwiedzki DM, Blankenship RE (2014) Intensity Dependence of the Excited State Lifetimes and Triplet Conversion Yield in the Fenna-MatthewsOlson Antenna Protein. J Phys Chem B 118:2058-2069. doi: 10.1021/jp411020a

Pšenčík J, Butcher SJ, Tuma R (2014) Chlorosomes: Structure, Function and Assembly. In: Hohmann-Marriott MF (ed) The Structural Basis of Biological Energy Generation. Springer Netherlands, Dordrecht, pp 77-109

Pšenčík J, Ma Y-Z, Arellano JB, et al (2003) Excitation Energy Transfer Dynamics and Excited-State Structure in Chlorosomes of Chlorobium phaeobacteroides. Biophys J 84:1161-1179 


\section{Chapter 5}

Psencik J, Mancal T (2017) Light-harvesting in green bacteria. In: Light-harvesting in Photosynthesis. CRC press

Reich NH, van Sark WGJHM, Turkenburg WC (2011) Charge yield potential of indoor-operated solar cells incorporated into Product Integrated Photovoltaic (PIPV). Renew Energy 36:642-647. doi: https://doi.org/10.1016/j.renene.2010.07.018

Ruban A V, Johnson MP, Duffy CDP (2011) Natural light harvesting: principles and environmental trends. Energy Environ Sci 4:1643-1650. doi: 10.1039/COEE00578A

Schlodder E, Hussels M, Çetin M, et al (2011) Fluorescence of the various red antenna states in photosystem I complexes from cyanobacteria is affected differently by the redox state of P700. Biochim Biophys Acta - Bioenerg 1807:1423-1431. doi: https://doi.org/10.1016/j.bbabio.2011.06.018

Tian L, Gwizdala M, van Stokkum IHM, et al (2012) Picosecond Kinetics of Light Harvesting and Photoprotective Quenching in Wild-Type and Mutant Phycobilisomes Isolated from the Cyanobacterium Synechocystis PCC 6803. Biophys J 102:1692-1700. doi: http://dx.doi.org/10.1016/j.bpj.2012.03.008

Tian L, Van Stokkum IHM, Koehorst RBM, et al (2011) Site, rate, and mechanism of photoprotective quenching in cyanobacteria. J Am Chem Soc 133:1830418311. doi: 10.1021/ja206414m

van Noort PI, Zhu Y, LoBrutto R, Blankenship RE (1997) Redox effects on the excited-state lifetime in chlorosomes and bacteriochlorophyll c oligomers. Biophys J 72:316-325. doi: https://doi.org/10.1016/S0006-3495(97)78670-3

van Stokkum IHM, van Oort B, van Mourik F, et al (2008) (Sub)-Picosecond Spectral Evolution of Fluorescence Studied with a Synchroscan StreakCamera System and Target Analysis. In: Aartsma TJ, Matysik J (eds) Biophysical Techniques in Photosynthesis. Springer Netherlands, Dordrecht, pp 223-240

Wang J, Brune DC, Blankenship RE (1990a) Effects of oxidants and reductants on the efficiency of excitation transfer in green photosynthetic bacteria. Biochim Biophys Acta - Bioenerg 1015:457-463. doi: https://doi.org/10.1016/00052728(90)90079-J

Wang J, Brune DC, Blankenship RE (1990b) Effects of oxidants and reductants on the efficiency of excitation transfer in green photosynthetic bacteria. Biochim Biophys Acta - Bioenerg 1015:457-463. doi: https://doi.org/10.1016/00052728(90)90079-J

Wen J, Zhang H, Gross ML, Blankenship RE (2009) Membrane orientation of the FMO antenna protein from Chlorobaculum tepidum as determined by mass spectrometry-based footprinting. Proc Natl Acad Sci 106:6134-6139. doi: 
Efficiency of excitation-energy trapping in the reaction center of the low-light adapted green photosynthetic bacterium Chlorobaculum tepidum

10.1073/pnas.0901691106

Wraight CA, Clayton RK (1974) The absolute quantum efficiency of bacteriochlorophyll photooxidation in reaction centres of Rhodopseudomonas spheroides. Biochim Biophys Acta - Bioenerg 333:246-260. doi: https://doi.org/10.1016/0005-2728(74)90009-7 
Chapter 5

Supplementary Information

Time-zero spectrum

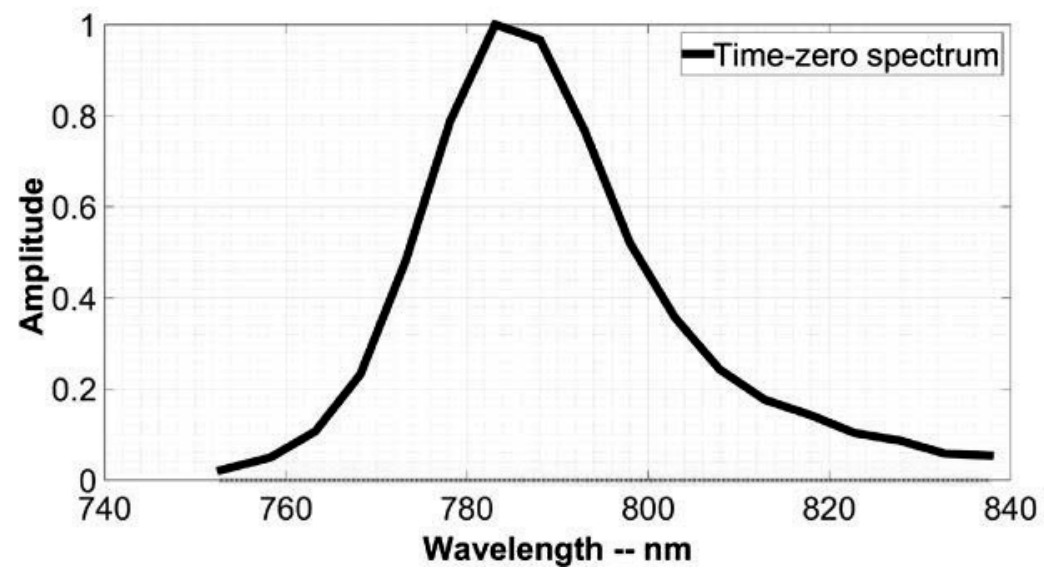

Figure S1: Time-zero spectrum at $77 \mathrm{~K}$ that is the sum of all the decay-associated spectra (DAS) in Figure 1c. 


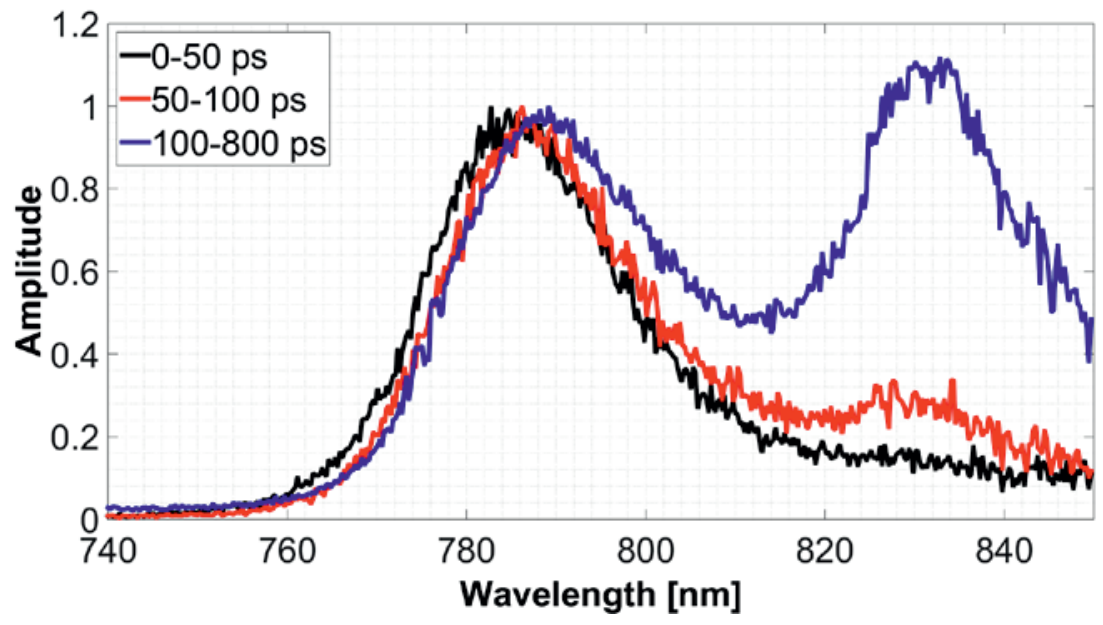

Figure S2: The $77 \mathrm{~K}$ fluorescence spectra of the cells integrated over various time periods after the excitation pulse. The integration time is shown in the legend.

\section{Time traces at $20^{\circ} \mathrm{C}$ and $77 \mathrm{~K}$ obtained from global analysis}

Figure S3 and Figure S4 show the time traces of each compartment in Figure 3 (77 K) and Figure $4\left(20^{\circ} \mathrm{C}\right)$, respectively. These time traces show the evolution of the population of each compartment over time. In both figures, the curve in magenta shows the population of the trapping state, which is not shown in the target model because it does not lead to fluorescence. At $20^{\circ} \mathrm{C}$ and $77 \mathrm{~K}$ the trapping state reaches a population of $\sim 0.88$ and $\sim 0.80$ respectively. Each compartment dissipates excitation energy with a rate of $(2.5 \mathrm{~ns})^{-1}$. 


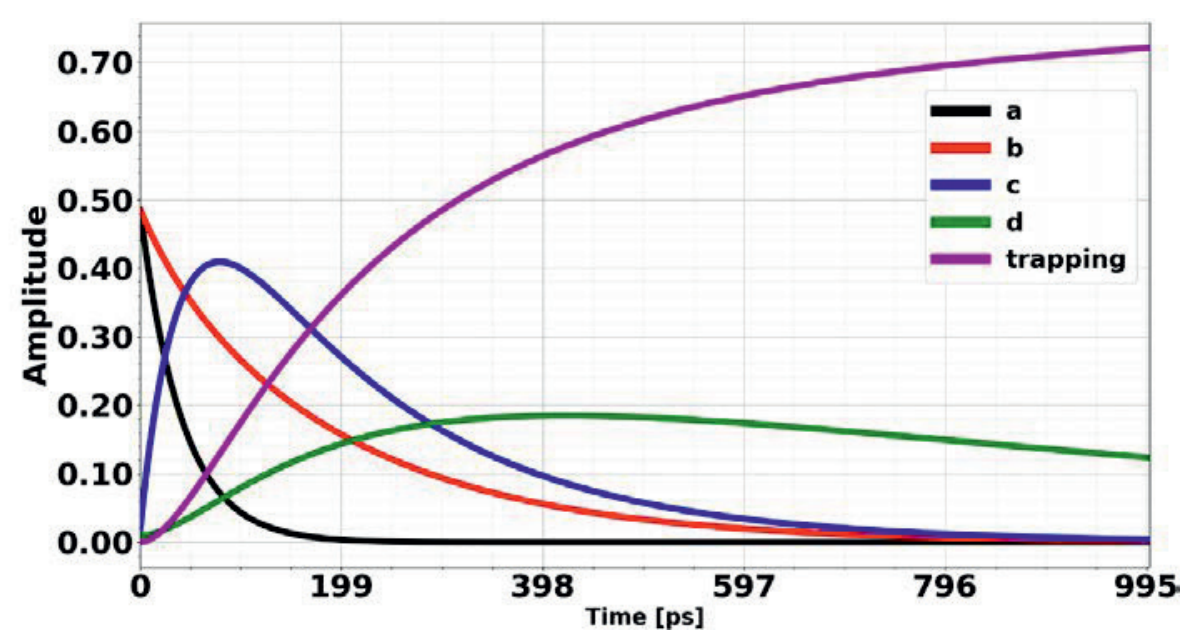

Figure S3: Time traces corresponding to the fractional population of compartments a-d in Figure $3(77 \mathrm{~K})$. The color of these time traces is the same as their corresponding SAS in Figure 3. The magenta curve shows the population of the charge-separated state that is not shown in the target model.

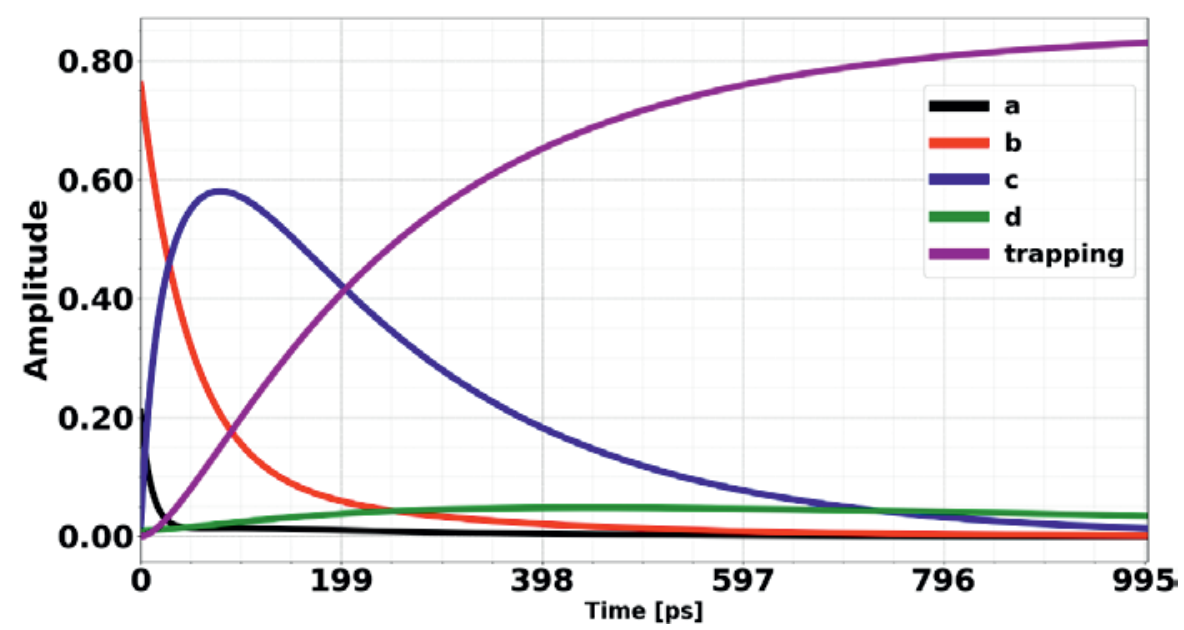

Figure S4: Time traces corresponding to the fractional population of compartments a-d in Figure $3\left(20^{\circ} \mathrm{C}\right)$. The color of these time traces is the same as their corresponding SAS in Figure 3. The magenta curve shows the population of the trapping state that is not shown in the target model. 
Efficiency of excitation-energy trapping in the reaction center of the low-light adapted green photosynthetic bacterium Chlorobaculum tepidum

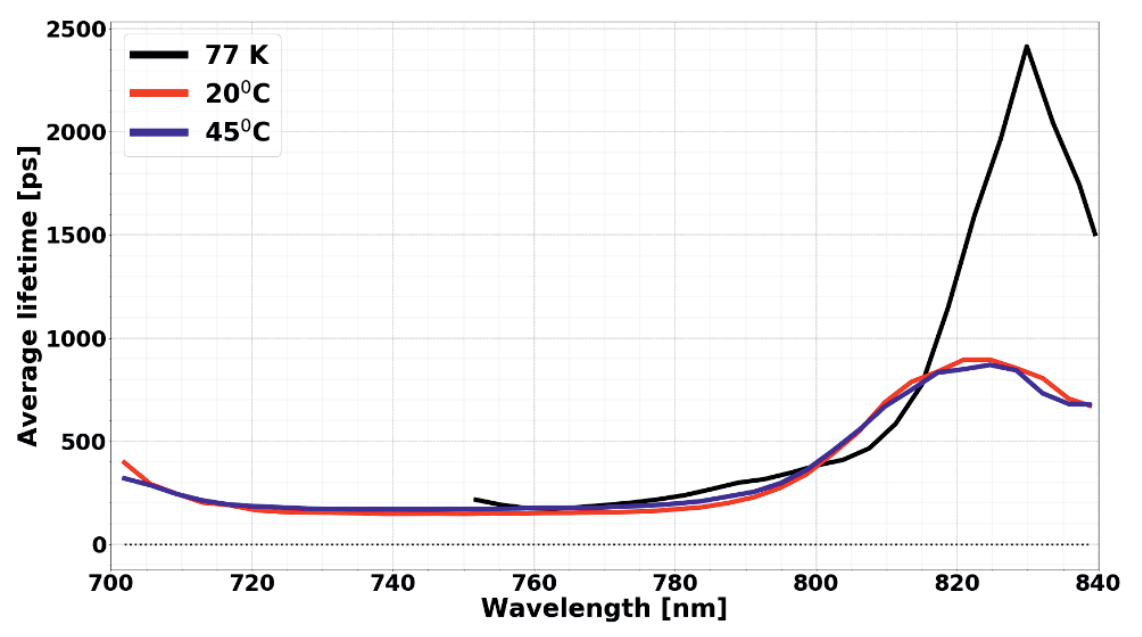

Figure S5: Average lifetime calculated at three different temperatures.

\section{RT detailed balance condition}

In the RT target analysis the rate of back transfer to the black compartment was a free parameter at first. After the first run of the target analysis this rate was fixed to the value obtained by the detailed-balance condition (see below). This procedure was performed until there was no need to update the back transfer rate to fulfil the detailed balance condition anymore. The detailed balance condition is given by the following relation between forward and backward energy transfer rates between the red and blue compartments, $k_{\text {red_blue }}$ and $k_{\text {blue_red, }}$ respectively:

$$
\frac{k_{\text {red_blue }}}{k_{\text {blue_red }}}=\frac{N_{\text {blue }}}{N_{\text {red }}} e^{\frac{E_{\text {red }}-E_{\text {blue }}}{k T}} \quad \text { Equation }
$$




\section{Chapter 5}

where $N_{\text {blue }}$ is the number of BChls a in the blue compartment and $N_{\text {red }}$ is the number of BChls $c$ in the red compartment (see Figure 3). $E_{\text {red }}$ and $E_{\text {blue }}$ are the energy levels of $\mathrm{BChl} c$ and $a$ in the red and blue compartments, $\mathrm{k}$ is the Boltzmann constant and $\mathrm{T}$ is the absolute temperature. A similar relation can be written for the black and blue compartments:

$$
\frac{k_{\text {black_blue }}}{k_{\text {blue_black }}}=\frac{N_{\text {blue }}}{N_{\text {black }}} e^{\frac{E_{\text {black }}-E_{\text {blue }}}{k T}}
$$

Equation

The energy of BChl $c$ in the red and black compartments is the same. Dividing Equation 1 by Equation 2 one obtains:

$$
k_{\text {blue_black }}=\frac{N_{\text {black }}}{N_{\text {red }}} \times \frac{k_{\text {blue_red }}}{k_{\text {red_blue }}} \times k_{\text {black_blue }}
$$

As described before, the best fit was obtained with the following ratio: $\frac{N_{\text {black }}}{N_{\text {red }}}=\frac{0.21}{0.76}$ 
Efficiency of excitation-energy trapping in the reaction center of the low-light adapted green photosynthetic bacterium Chlorobaculum tepidum 
Chapter 5

162 


\section{6}

General Discussion 
Introduction

Photosynthesis is the natural process of converting light energy to chemical energy, which is used directly or indirectly by almost all living organisms on earth. Photosynthetic organisms use their light-harvesting antenna to absorb light. The absorption of light excites the pigments in the antenna. The excitation energy then travels through the antenna to reach the reaction centers (RCs), where during a process called charge separation an electron is donated to electron-carriers. In oxygenic photosynthetic organisms, two photosystems (PSI and PSII) coexist, each of which has its own antennas and RCs. Intersystem-electron carriers transport electrons from the RCs of PSII to the cytochrome b6f complex. The RCs of PSII split water to replace the donated electron, and the RCs of PSI replace the donated electron by accepting electrons from electron-carriers, which transport electrons from the cytochrome b 6 complex to PSI. RCs of PSI donate an electron to ferredoxin, which in turn forms the ferredoxin-NADP reductase complex. The ferredoxin-NADP reductase complex is bound to $\mathrm{PSI}$, which produces the reduced electron carrier nicotinamide adenine dinucleotide phosphate (NADPH). Oxidation of electroncarriers in the cytochrome bff and water splitting by RCs of PSII create a proton gradient across the membrane, which is used by a protein complex called ATP. synthase to produce adenosine triphosphate (ATP). ATP in turn is used in the CalvinBenson-Bassham cycle to produce biomass.

Photosynthetic organisms live in various environments. When the organisms are exposed to high-light intensities they utilize their photo-protection mechanisms to survive the adverse side-effects of these high-light intensities. Some environments on the other hand provide the organism with little light and the organisms have to develop a larger light-harvesting antenna to harness as much light as possible.

The light-harvesting antennas of photosynthetic organisms can differ substantially, depending on the spectral composition of the light in a specific habitat. In most antennas, pigments are embedded in a protein scaffold. The structure of these proteins affects the absorption of light and more importantly, the efficiency of excitation energy transfer (EET). An example of such an antenna is the phycobilisome (PBS) in cyanobacteria. Other light-harvesting antennas are self- 
aggregates of pigments without involvement of proteins such as the chlorosomes of green sulfur Chlorobaculum tepidum.

In steady-state fluorescence spectroscopy, the fluorescence is recorded and accumulated over time, which only results in steady-state spectra without any information about the time-scales of EET and charge separation. One method to study EET in photosynthetic organisms is time-resolved fluorescence spectroscopy. A laser pulse with the proper wavelength excites the light-harvesting antennas, which creates an excited-state population. This excited-state population subsequently disappears again via several mechanisms. An important one is charge separation in the RCs and another one is radiative emission of the excited state energy (fluorescence). Quenching of excitation energy by photo-protection mechanisms also shortens the fluorescence lifetime. The fluorescence lifetime of a group of pigments fluorescing at a specific wavelength is shortened by EET to another group of pigments, which fluoresce at longer wavelengths.

Over the years, many crystal structures of a large variety of antenna complexes have been resolved in ever greater detail. Time-resolved fluorescence spectroscopy provides a means to study the functioning of these antenna complexes. It also allows comparing the function of these complexes in their crystal and solution forms. The comparison allows us for instance to determine whether the structures resolved by $\mathrm{x}$-ray crystallography are identical to the in vivo structures.

The advancement in experimental equipment and data analysis methods has allowed us to study adaptation mechanisms in more detail and also to revisit some of the already studied phenomena with new approaches to resolve the contradictory observations obtained with more restrictive approaches such steady-state fluorescence spectroscopy.

In the next sections, I describe in vivo measurements on entire organisms. I combined time-resolved spectroscopy and data analysis to study regulation (statetransitions or STs) and photoprotection (non-photochemical quenching or NPQ) phenomena in two different strains of cyanobacteria. I have demonstrated that STs exhibit PSII quenching even at low light intensities. NPQ in plants results in PSII quenching too, but NPQ only occurs at high light intensities. The particular 
photoprotection mechanism studied in this thesis is induced by drought to protect the organism against high light intensity and it occurs in the PBSs.

I also applied the same methods to study excitation energy transfer in green sulfur bacteria. I performed time-resolved measurements on whole cells at both room temperature and $77 \mathrm{~K}$. Previous studies were performed on either isolated chlorosomes, isolated RCs and Fenna-Matthews-Olson protein (FMO), or only at 77 $\mathrm{K}$. My in vivo room temperature time-resolved measurements, in combination with the measurements performed at $77 \mathrm{~K}$ allowed me to estimate the efficiency of trapping in vivo and to assess the effect of temperature on this efficiency.

\section{Excitation energy transfer in isolated allophycocyanin trimers}

PBSs, which absorb mainly green-orange light, form the main part of the lightharvesting antennas of cyanobacteria. PBSs are organized as stacks of phycobiliproteins (PBPs), which are held together by linker proteins. PBPs affect the spectral characteristics of the phycocyanobilin (PCB) pigments they contain. This influence plays an important role in the direction, rate and efficiency of EET between chromophores. The core of PBSs consists of allophycocyanin (APC) to which the phycocyanin $(\mathrm{PC})$ rods are attached.

APC is a red-light absorbing PBP that binds PCB chromophores. Each monomer in an APC trimer has two subunits called $\alpha$ - and $\beta$-subunits and each subunit contains one PCB. Upon trimer formation, one PCB of an $\alpha$-subunit comes close to the PCB of a $\beta$-subunit in another monomer to form a low- and high-energy pair.

I performed time-resolved spectroscopy measurements on APC trimers in crystal form and in solution. To determine the structure of APC trimers they have to be in crystalline form. However, the structure of APC trimers in crystal form could be different from the in vivo form. The structure of these protein-pigment complexes affect EET among their pigments, and this EET can be studied by time-resolved spectroscopy to observe any possible structural difference.

The experimental data were analyzed by global and target analysis (van Stokkum et al. 2004), which yielded decay-associated (DAS) and species-associated (SAS) spectra. These spectra and the obtained EET rates were used to compare APC in 166 
crystal form and in solution to determine if the structure of APC in crystal form is the same as APC in solution.

According to the literature (for an overview see van Amerongen, H., Valkunas, L., \& van Grondelle 2000), the EET from a $\beta$-subunit of one monomer to an $\alpha$-subunit of the adjacent monomer occurs within $\sim 1$ ps. However, I also observed much slower EET that occurs in tens of picoseconds. Based on global and target analyses I concluded that not all the closely spaced PCB pairs include one high- and one lowenergy configuration. Around $10 \%$ of these pairs include two high-energy configurations and the EET observed in my measurements is the EET between these high energy pairs and other high- and low-energy PCB pairs. The resolved fluorescence lifetimes for APC in crystal form are significantly shorter than the corresponding lifetimes of APC in solution, which suggests the structure of APC in crystal and solution form is not identical.

Dual conformational states also occur in other photosynthetic complexes such as PC (Gwizdala et al. 2018) and the light-harvesting complex LHCII of green plants (Pascal et al. 2005; Krüger et al. 2010). For instance, one of the conformations of LHCII can serve for light harvesting in low-light conditions and another conformation causes quenching of the excited state in high-light conditions. PC rods are able to transfer excitation energy directly to PSI in their red-shifted conformation or to the core of PBS in their other conformation.

\section{State transitions}

Cyanobacteria use PBSs to absorb mainly green or orange light, while chlorophyll (Chls) a of PSI and PSII absorb mainly blue and red light. Excitation energy delivered to the RC of PSII causes the RC to perform charge separation. Inter-system electron carriers, which are reduced after accepting an electron from the RC of PSII, transport the released electrons to the cytochrome b6f complex and become oxidized and from there plastocyanin delivers electrons to PSI. The ratio of oxidized/reduced electron carriers depends on the light spectrum. For example, in state I (induced by blue or red light) the electron carriers are more oxidized and in state II (induced by greenorange light) they are more reduced. State I (state II) is characterized by a high (low) ratio of PSII/PSI fluorescence peaks. 
Several models are currently used to describe state transitions. One possible mechanism is spill-over of excitation energy from PSII to PSI in state II without the involvement of PBSs. PSII either receives excitation energy directly via light absorption by $\mathrm{Chl}$ a or indirectly via PBSs excitation. According to the spill-over model, PSII transfers part of its excitations to PSI in state II, which decreases the ratio of PSII/PSI emission. In this way, PSI quenches the excess excitations of PSII and uses them to restore the balance of charge separation in both photosystems. This model explains the observed low and high ratio of PSII/PSI fluorescence peaks in state I and II, respectively. A combination of the spill-over model and a slight PBSs movement has also been proposed (McConnell et al. 2002). In this model, PBSs have a stable connection to PSII and a variable connection to PSI. In state II, the rate of EET from PSII to PSI and from PBSs to PSI increases, which results in a lower ratio of PSII/PSI emission. Part of the excitation of PSII is quenched directly by PSI and the amount of EET from PBSs to PSII decreases because of an increase in the rate of EET to PSI.

According to the current models, PSI emission must rise in state II. While some studies do show an increase of PSI emission in state II (Bruce et al. 1989; Salehian and Bruce 1992), some other studies demonstrate that PSI emission remains the same in state I and II (Olive et al. 1997; El Bissati et al. 2000; McConnell et al. 2002). These observations were based on steady-state measurements. It could be that the decreasing emission spectrum of PSII in state II overlaps with the rising spectrum of PSI, which would mask any change of the PSI emission. Time-resolved measurements allow to distinguish the spectra of different complexes on different time scales and minimize the mentioned effect.

I performed $77 \mathrm{~K}$ fluorescence time-resolved and steady-state spectroscopy on whole cells of Synechocystis PCC 6803 and Synechococcus elongatus 7942, which were brought to state I or II, immediately before freezing the samples. At $77 \mathrm{~K}$ it is easier to observe PSI emission and the spectra of other fluorescing species are distinguished better from each other due to a sharpening of the fluorescence bands at cryogenic temperatures. I used $430 \mathrm{~nm}$ and $577 \mathrm{~nm}$ excitation to excite Chls a and PBSs, respectively. To validate the time-resolved measurements, albeit in an indirect way, the steady-state spectra reconstructed from time-resolved 
measurements were compared with the measured steady-state spectra. Both the measured and reconstructed steady-state spectra showed similar differences between state I and II.

Global analysis of the $77 \mathrm{~K}$ fluorescence data revealed that the emission of PSII is quenched in state II as compared to state I. Because it was also found that PSI emission is not different for states I and II, it could be concluded that PSII excitations are not quenched by PSI, in disagreement with the spill-over model. Analysis of the data obtained upon PBSs excitation in addition revealed a partial detachment of PBSs from photosystems in state I.

The extent of PSII quenching depends on the strain of cyanobacteria. For example, Synechocystis PCC 6803 also exhibits PSII quenching, but on a much smaller scale than Synechococcus elongatus 7942. Our unpublished results obtained by timeresolved spectroscopy on Leptolyngbya ohadii show that in the desiccated state PSII is quenched. Whereas quenching of PSII in Synechococcus elongatus 7942 occurs at low-light intensity upon induction of state II, in Leptolyngbya ohadii PSII is quenched in the case of drought.

\section{Excitation energy quenching in phycobilisomes}

If a photosynthetic organism is exposed to high light, the many absorbed photons lead to closure of the reaction centers and charge separation, which leads to the disappearance of excitations, stops. As a result, the excited state lifetime of RC becomes long and intersystem crossing occurs with a high probability, especially in the closed RCs themselves. The triplet excited state of RC produces singlet oxygen, which is damaging the cells. To prevent this damage, it is necessary to quench the excess excitations so that RC triplet formation and singlet oxygen production reduces substantially.

Leptolyngbya ohadii is a cyanobacterium living in the desert, which also has to cope with high-light intensities and drought. These cyanobacteria are able to quench the excess energy at the PBS level; however, the mechanism of quenching was unknown. 
I performed $77 \mathrm{~K}$ and room temperature time-resolved fluorescence measurements on whole cells of Leptolyngbya ohadii, either in the desiccated (dry) or hydrated state. The global analysis yielded the DAS and decay lifetimes in desiccated and hydrated states.

In the desiccated state, the EET between PBS components is reduced and the fluorescence decay time is shortened, which demonstrates that the excitation energy within PBSs is severely quenched. The quenching is accompanied by a red shift of the emission spectra and small-angle $X$-ray and neutron scattering demonstrate that these changes coincide with a loss of the ordered PBS structure.

Based on the experimental results we propose a model, in which the organized rod structure of PBSs in the hydrated state supports efficient directional EET but in the desiccated state this organization is lost, giving rise to more random aggregates, which are characterized by enhanced quenching.

\section{Excitation energy transfer in Chlorobaculum tepidum}

The green sulfur bacterium Chlorobaculum tepidum uses chlorosomes as its lightharvesting antennas. Chlorosomes with typical lengths of $100-200 \mathrm{~nm}$ and diameters of 40-60 nm are mainly made of self-aggregates of bacteriochlorophyll (BChl) $c, e$, or $d$ (only one BChl type per species) (Oostergetel et al. 2010). The chlorosomes are encapsulated by a lipid monolayer, in which the baseplate is embedded. The baseplate consists of the CsmA protein, to which BChl a is bound. The excitation energy absorbed by the $\mathrm{BChl}$ aggregates in the chlorosomes is transferred via the baseplate to the BChl-a-binding FMO complexes. From there the excitation energy is transferred to the RCs, where charge separation occurs.

Photosynthetic organisms have adapted to live in different environments. Some organisms live in habitats with high-light intensity such as the cyanobacterium Leptolyngbya ohadii, whereas others such as C. tepidum live in low-light conditions. Having a large antenna helps $C$. tepidum to harvest more light. However, it is expected that EET to the RCs occurs on a longer time scale when the antenna size increases, which in turn means that the probability of losing the excitation energy increases. The volume of chlorosomes is at least $\sim 500$ times larger than that of a 
PSII monomer and one might maybe expect the efficiency of EET to be low in green sulfur bacteria. The efficiency of EET in green sulfur bacteria like $C$. tepidum has always been a matter of debate. To study the efficiency of trapping in $C$. tepidum, I performed time-resolved fluorescence measurements on whole cells at $77 \mathrm{~K}$ and room temperature with extremely low laser powers. The results indicate that the time scale of trapping (the average time it takes for the excitation energy to reach the $\mathrm{RCs}$ ) in C. tepidum is comparable to that of cyanobacteria, which have a much smaller antenna. This indicates that the design of chlorosomes as a self-aggregate of pigments makes EET extremely fast. The comparison of room temperature and $77 \mathrm{~K}$ measurements indicate that the temperature does not affect this time scale considerably. Using global and target analyses I estimated the efficiency to be $\sim 80 \%$.

In this thesis, I used time-resolved fluorescence spectroscopy to study various aspects of photosynthesis. PBSs are the major light-harvesting antennas of cyanobacteria and I studied APC trimers, which lie in the core of PBS. I performed measurements on two different strains of cyanobacteria to study photoprotection and regulation of excitation energy. I also performed $77 \mathrm{~K}$ and in vivo measurements on green sulfur bacteria $C$. tepidum to study the efficiency of EET.

It was revealed, that the protein structure of APC trimers have at least two different structures, and this structure is slightly different between crystal form and the functional form. The study of state-transition concluded that spill-over of excitation energy from PSII to PSI does not play a role in state-transitions and PSII is quenched in state II without the involvement of PSI. The work on desert cyanobacteria L. ohadii resulted in a model for photoprotection in L. ohadii cells, in which the disruption of the organization of PC rods in the PBSs leads to quenching. The $77 \mathrm{~K}$ and in vivo measurements performed on the green sulfur bacterium $C$. tepidum showed that the efficiency of EET in this bacterium does not depend significantly on temperature and while its antenna is much larger than that of cyanobacteria, the time scale of EET is comparable. 


\section{References}

Bruce D, Brimble S, Bryant DA (1989) State transitions in a phycobilisome-less mutant of the cyanobacterium Synechococcus sp. PCC 7002. Biochim Biophys Acta - Bioenerg 974:66-73. doi: https://doi.org/10.1016/S00052728(89)80166-5

El Bissati K, Delphin E, Murata N, et al (2000) Photosystem II fluorescence quenching in the cyanobacterium Synechocystis PCC 6803: involvement of two different mechanisms. Biochim Biophys Acta - Bioenerg 1457:229-242. doi: http://dx.doi.org/10.1016/S0005-2728(00)00104-3

Gwizdala M, Krüger TPJ, Wahadoszamen M, et al (2018) Phycocyanin: One Complex, Two States, Two Functions. J Phys Chem Lett 9:1365-1371. doi: 10.1021/acs.jpclett.8b00621

Krüger TPJ, Novoderezhkin VI, Ilioaia C, van Grondelle R (2010) Fluorescence Spectral Dynamics of Single LHCII Trimers. Biophys J 98:3093-3101. doi: https://doi.org/10.1016/j.bpj.2010.03.028

McConnell MD, Koop R, Vasil'ev S, Bruce D (2002) Regulation of the distribution of chlorophyll and phycobilin-absorbed excitation energy in cyanobacteria. A structure-based model for the light state transition. Plant Physiol 130:12011212. doi: 10.1104/pp.009845

Olive J, Ajlani G, Astier C, et al (1997) Ultrastructure and light adaptation of phycobilisome mutants of Synechocystis PCC 6803. Biochim Biophys Acta Bioenerg 1319:275-282. doi: https://doi.org/10.1016/S0005-2728(96)00168-5

Oostergetel GT, van Amerongen H, Boekema EJ (2010) The chlorosome: a prototype for efficient light harvesting in photosynthesis. Photosynth Res 104:245-255. doi: 10.1007/s11120-010-9533-0

Pascal AA, Liu Z, Broess K, et al (2005) Molecular basis of photoprotection and control of photosynthetic light-harvesting. Nature 436:134-137

Salehian O, Bruce D (1992) Distribution of excitation energy in photosynthesis: quantification of fluorescence yields from intact cyanobacteria. J Lumin 51:91-98. doi: https://doi.org/10.1016/0022-2313(92)90021-Z

van Amerongen, H., Valkunas, L., \& van Grondelle R (2000) Photosynthetic Excitons. World Scientific

van Stokkum IHM, Larsen DS, van Grondelle R (2004) Global and target analysis of time-resolved spectra. Biochim Biophys Acta - Bioenerg 1657:82-104. doi: http://doi.org/10.1016/j.bbabio.2004.04.011 
Summary 
In this thesis, I used time-resolved fluorescence spectroscopy to study regulation of excitation energy transfer (EET) and photoprotection in cyanobacteria and the green sulfur bacterium Chlorobaculum tepidum.

Chapter 2 describes the study of EET in APC trimers, which are part of the antenna of cyanobacteria in crystal and functional forms. It is concluded that the same protein has at least two different structures in APC trimers and that the structure of APC trimers in crystal form deviates to some extent from the structure of APC in protein solution.

Chapter 3 presents the study of state transitions in the cyanobacterium Synechococcus elongatus 7942. It is concluded that in state II photosystem II (PSII) is quenched and the spill-over of excitation energy from PSII to PSI is not involved in state transitions. It is also shown that in state I some of the phycobilisomes (PBSs) detach from both photosystems.

Chapter 4 presents a model of photoprotection in the cyanobacterium Leptolyngbya ohadii, which is normally living in the desert. In the desiccated state, excitation energy in the PBS is severely quenched as compared to the hydrated state. It is concluded that the quenching is due to the loss of the organized structure of PC rods in the dry state.

Chapter 5 present the study of EET in the green sulfur bacterium C. tepidum. It is demonstrated that the efficiency of EET does not depend significantly on the temperature and that the time scale of excitation energy trapping in the reaction centers is comparable with other much smaller photosynthetic systems such as that of cyanobacteria. 
Chapter 7

Summary 


\section{Acknowledgement}

I would like to take the opportunity to express my appreciation and gratitude towards people who supported me during my PhD.

First of all, I would like to thank my daily supervisor prof. Herbert Van Amerongen. He gave me the opportunity to perform research on a new and multidisciplinary subject in a friendly environment. He gave me lots of freedom to explore and perform research on topics which I was interested in. While I enjoyed my freedom to work on different topics and ideas, his guidance and support prevented me from getting lost in the wrong direction.

I would also like to thank my co-supervisor prof. Paul C. Struik for his guidance and support.

I thank Rob who taught me a lot about spectroscopy and experimental setups.

Moreover, I thank Diana, Emilie, Paul, Herbert, Leeat, Ravi, Arjen, Nir, Rob, and Jakub for their presence, help and contribution to our common manuscripts. I also thank all other co-authors and people whom I collaborated with. Their help made performing research easier and an enjoyable activity.

I appreciate Arjen for his technical support and Cor for helping me with the lab supplies. I appreciate Netty's help when I arrived in the Netherlands and her help with many administrative issues.

I appreciate the help of Ahmad and Koen as my paranymphs. Thank you all biophysics members who created a lively and friendly environment: Emilie, Johannes ,Mattia, Koen, Tatiana, Ahmad, Julia, Shazia, Shanthi, Caner, Ievgeniia, Alena, Elena, Olga, Fugui, Daan, Raquel, Du, Rob, Netty, Arjen, Carel, Henk, Frank, and John.

I express my highest gratitude toward my beloved wife, Helen, who always encouraged and supported me. Her love and company gave me confidence and energy to overcome challenges in life and PhD.

I also appreciate my father (Rahim), and my sisters (Sara and Yasaman) who always supported and encouraged me. My special thanks go to my mother (Sosan) who always gave me confidence and support. 


\section{Education Statement of the Graduate School \\ Experimental Plant Sciences}

Issued to:

Date:

Group:

University:
Reza Ranjbar Choubeh

19 November 2018

Laboratory of Biophysics

Wageningen Univeristy \& Research

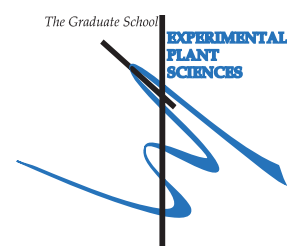

State transition and NPQ in cyanobacteria

- Writing or rewriting a project proposal

- Writing a review or book chapter

- MSc courses

Laboratory use of isotopes $\underline{\text { date }}$

20 Sep 2014

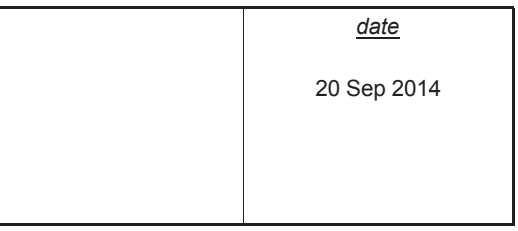

Subtotal Start-Up Phase

$1.5^{*}$

\section{2) Scientific Exposure}

- EPS PhD student days

EPS PhD Student Days 'Get2Gether'

EPS PhD Student Days 'Get2Gether'

- EPS theme symposia

EPS Theme 3 Symposium 'Metabolism and Adapatation', Amsterdam, NL

EPS Theme 3 Symposium 'Metabolism and Adaptation', Wageningen, NL

\section{National meetings (e.g. Lunteren days) and other National Platforms}

BioSolar Cells Cluster Meeting, NL

BioSolar Cells Cluster Meeting, NL

BioSolar Cells Cluster Meeting, NL

BioSolar Cells Cluster Meeting, NL

BioSolar Cells Annual Meeting 2014, NL

BioSolar Cells Annual Meeting 2015, NL

Dutch Biophysics 2013, Veldhoven, NL

Dutch Biophysics 2014, Veldhoven, NL

Dutch Biophysics 2015, Veldhoven, NL

Dutch Biophysics 2017, Veldhoven, NL

Physics@Veldhoven 2015, Veldhoven, NL

Physics@Veldhoven 2017, Veldhoven, NL

Physics@Veldhoven 2018, Veldhoven, NL

- Seminars (series), workshops and symposia

Science week: What is life?, Wageningen, NL

Conference: Models, improving photosynthesis and better crop yields, Wageningen, NL

Symposium: 'Food for Future: Nutrition, Health and Novel Food \& Consumer Behaviour', Wageningen, NL

Symposium: Molecules@ @URk, Wageningen, NL

Seminar: 'How Molecules can WURC for you?! The Rise and Development of the Mechanical Bond',

Prof. Dr. Fraser Stoddart

Seminar: 'Artificial Metalloenzymes: Challenges and Opportunities', Prof. Dr. Thomas Ward

Seminar: 'Bioorganometallic Chemistry: Synthetic Strategies and Biomedical Applications for Metal-

Peptide Bioconjugates', Prof. Dr. Nils Meztler-Nolte

Seminar: 'Tools for Quantification of Signaling Complexes Using Fluorescence Correlation Spectroscopy',

Dr. Mark Hink

Seminar: 'Exploring the Galaxy of Nanostructured Polymer Materials with Superresolved Fluorescence Imaging', Dr. Dominik Wöll

Seminar: 'Bacteria and the Future of Agriculture: From Sequence to Function', Dr. Asaf Levy

Seminar: 'Pelargonidin in Flowers - Why Not? Gerbera and Petunia Flowers Block Pelargonidin

Biosynthesis in a Different Way', Dr. Teemu Teeri

Seminar: 'How do living cells work?', Prof. Dr. Wilhelm Huck

Seminar: Wageningen Molecular Life Sciences Seminar, Prof. Dr. Hans Aerts

Seminar: 'Using Light to Dissect and Direct Intracellular Transport', Dr. Lukas Kapitein

Seminar: 'The De-Novo Synthesis of Life', Prof. Dr. Sijbren Otto

Seminar: 'Algal Genomics and Transcriptomics in the Service of Evolution and Ecology', Prof. Dr. Charles

\section{- Seminar plus}

- International symposia and congresses

Light Harvesting Satellite Meeting, Egmond aan Zee, NL

17th International Congress on Photosynthesis Research, Maastricht, NL

17th European Conference on the Spectroscopy of Biological Molecules (ECSBM), Amsterdam, NL

\section{- Presentations}

Poster: BioSolar Cells Annual Meeting 2014

Poster: BioSolar Cells Annual Meeting 2015

Poster: Dutch Biophysics 2014

Poster: Dutch Biophysics 2017

\section{$\underline{\text { date }}$}

28-29 Jan 2016 09-10 Feb 2017

23 Feb 2016

14 March 2017

04 Dec 2013

24 Jun 2014

04 Dec 2014

18 Jun 2015

23 Jun 2014

17 Jun 2015

30 Sep - 01 Oct 2013

29-30 Sep 2014

28-29 Sep 2015

01-02 Oct 2017

20-21 Jan 2015

17-18 Jan 2017

23-24 Jan 2018

12-14 Mar 2018

21 Jun 2018

23 Jun 2018

08 Feb 2017

17 Nov 2017

19 Dec 2017

18 Jan 2018

14 Feb 2018

14 Feb 2018

22 Feb 2018

14 Mar 2018

15 Mar 2018

19 Apr 2018

03 May 2018

17 May 2018

18 Jun 2018

04-07 Aug 2016

07-12 Aug 2016

11-14 Sep 2017

23 June 2014

17 Jun 2015

29-30 Sep 2014

01-02 Oct 2017 
Poster: 17th International Congress on Photosynthesis Research

Poster: 17th European Conference on the Spectroscopy of Biological Molecules (ECSBM)

Poster flash: Light Harvesting Satellite Meeting

07-12 Aug 2016

11-14 Sep 2017

04-07 Aug 2016

23 Oct 2014

:

18 Jun 2015

Talk: BioSolar Cells Cluster Meeting

10 Feb 2017

- IAB interview

- Excursions

Subtotal Scientific Exposure

$24.1^{\text {* }}$

\section{3) In-Depth Studies}

- EPS courses or other PhD courses

COST PHOTOTECH Training School: 'Advanced Laser Spectroscopy in Green Phototechnology',

Szeged, Hungary

Course: 'Introduction to R for Statistical Analysis', Wageningen, NL

Course: 'Linear Models', Wageningen, NL

Course: 'Generalized Linear Models', Wageningen, NL

Course: 'Mixed Linear Models', Wageningen, NL

date

Journal club

- Individual research training

18-23 Oct 2014

08-09 May 2017

07-09 Jun 2017

26-27 Jun 2017

29-30 Jun 2017

Subtotal In-Depth Studies

4.2 *

4) Personal Development

- Skill training courses

Course: NWO Course 'Taking Charge of Your PhD Project', Utrecht, NL

Course: NWO Course 'Dutch Welcome', Utrecht, NL

Course: 'The Essentials of Scientific Writing and Presenting', Wageningen, NL

Course: 'Brain Training', Wageningen, NL

Workshop: 'Scientific publishing', Wageningen, NL

Workshop: 'Transferable Skills', Wageningen, NL

- Organisation of PhD students day, course or conference

Membership of Board, Committee or PhD council

$\underline{\text { date }}$

19 Nov \& 03 Dec 2013

27 Feb - 05 Mar 2014

13-25 Nov 2015

20 Sep 2017

15 Oct 2015

28 Jun 2018

Subtotal Personal Development

$4.1^{*}$

Herewith the Graduate School declares that the $\mathrm{PhD}$ candidate has complied with the educational requirements

set by the Educational Committee of EPS which comprises of a minimum total of 30 ECTS credits.

${ }^{*}$ A credit represents a normative study load of 28 hours of study.

The Netherlands Organization for Scientific Research (NWO) financially supported the research described in this thesis.

This project was carried out within the research programme BioSolar Cells, cofinanced by the Dutch Ministry of Economic Affairs.

Research presented in this thesis was carried out at the Laboratory of Biophysics, Wageningen University \& Research, Wageningen, The Netherlands 